\title{
Analyse der Glättung rauer Oberflächen durch Dünnschichtdeposition
}

\author{
Dissertation \\ zur Erlangung des Doktorgrades der Mathematisch- \\ Naturwissenschaftlichen Fakultäten \\ der Georg-August-Universität zu Göttingen
}

vorgelegt von

Johanna Röder

aus Kassel

Göttingen 2009 
D 7

Referent: Prof. Dr. H.-U. Krebs

Korreferent: Prof. Dr. H. Hofsäss

Tag der mündlichen Prüfung: 23.06.2009 


\section{Inhaltsverzeichnis}

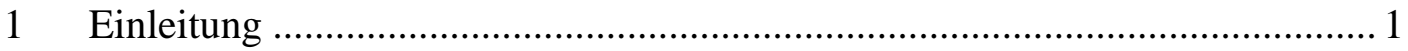

2 Probenherstellung und Charakterisierung ....................................................... 4

$2.1 \quad$ Gepulste Laserdeposition (PLD) ......................................................... 4

$2.2 \quad$ Rasterkraftmikroskopie (AFM) ………………................................... 8

2.3 Transmissionselektronenmikroskopie (TEM) .......................................... 9

$2.4 \quad$ Rasterelektronenmikroskopie (REM) .................................................... 10

$2.5 \quad$ Röntgenanalyse.............................................................................. 10

2.6 Schichtdickenbestimmung mittels Profilometrie und Ellipsometrie........ 11

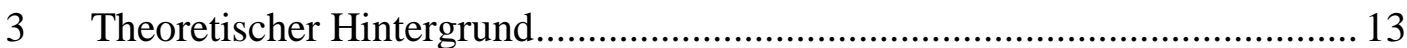

3.1 Rauigkeiten und Korrelationsfunktionen.................................................... 13

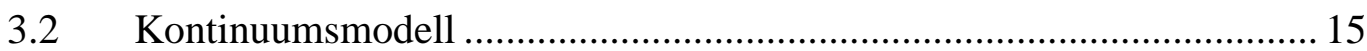

3.3 Oberflächenprozesse bei der Schichtdeposition mit PLD .......................... 17

3.4 Frequenzabhängigkeit der Glättung.......................................................... 19

3.5 Entwicklung der Rauigkeit ..................................................................... 24

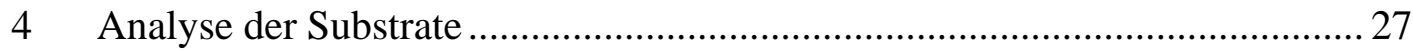

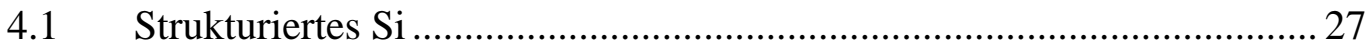

4.2 Statistische Oberflächenstrukturen ............................................................ 30 
1 Einleitung

$5 \quad$ Materialspezifische Glättung welliger Oberflächenstrukturen........................ 35

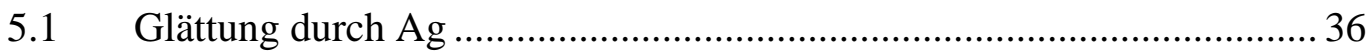

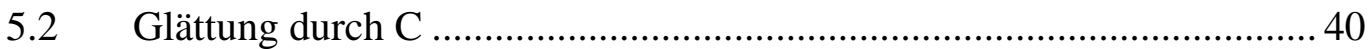

5.3 Glättung durch PMMA …..................................................................... 44

5.4 Glättung durch PC …...................................................................... 52

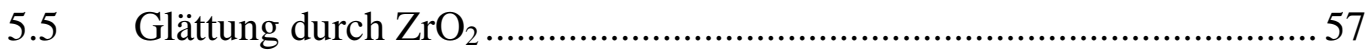

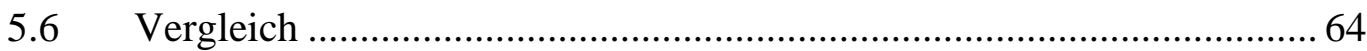

6 Frequenzabhängige Glättung statistischer Oberflächenstrukturen................. 67

6.1 Schichtdickenabhängigkeit der Glättung.............................................6 67

6.2 Abhängigkeit von der Oberflächenbeschaffenheit des Substrats ............ 75

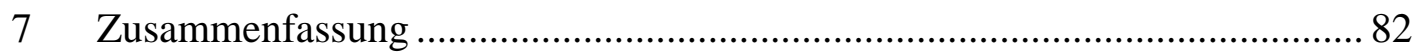

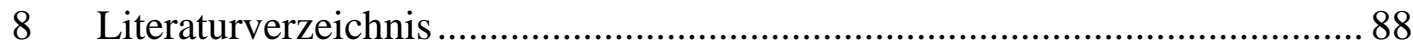




\section{Einleitung}

Die Beschaffenheit von Oberflächen und Grenzflächen spielt heutzutage eine wichtige Rolle bei vielen verschiedenen technischen Anwendungen. Eigenschaften wie z.B. Abriebfestigkeit, Schutz gegen Korrosion, Glanz oder Härte müssen kontrolliert werden, will man alltägliche Dinge wie Lacke für Möbel oder Autos, Beschichtungen für Spiegel, Brillengläser oder Geschirr und ähnliches von hoher Qualität herstellen. Hierbei ist nicht nur die Kenntnis der Oberflächenmorphologie und -rauigkeit, sondern vor allem die experimentelle Beeinflussung und kontrollierte Einstellung dieser Parameter von großem Interesse, z.B. bei der fortschreitenden Miniaturisierung von elektronischen Bauelementen. Weitere Beispiele für die Bedeutung dieser Größen in der Anwendung sind Schichtpakete als Röntgenspiegel [Sava91]. Hier führt die Minimierung der Grenzflächenrauigkeit und die Vermeidung von kumulativen Rauigkeiten zu einer starken Zunahme der Reflektivität und zur deutlichen Verbesserung der Auflösung der Abbildungsoptik durch Verringerung des störenden Untergrundes [Chri88]. Auch bei Magnetowiderstandssystemen (MR, GMR, TMR) hängt die Streuung der Elektronen stark von der Grenzflächenbeschaffenheit ab. Eine gezielte Einstellung der Rauigkeit kann dabei zu einer Zunahme des GMR-Effekts führen [Full92, Faup02].

Die Struktur einer Oberfläche kann systematisch verändert werden, indem man dynamische Prozesse ablaufen lässt, wie beispielsweise die Bestrahlung einer Oberfläche mit energetischen Ionen oder das Wachstum einer dünnen Schicht. Hierbei finden auf atomarer Skala verschiedene Mechanismen wie Diffusion, Desorption, Sputtererosion, lokales Aufschmelzen oder ballistisches Glätten statt [Bara95, Herr50, Mull57, Mose05], die in der Summe sowohl eine aufrauende als auch eine glättende Wirkung haben können. Im Falle der Ionenbestrahlung von Oberflächen wurde das Zusammenspiel dieser Prozesse 
bereits vielfach in Abhängigkeit von Parametern wie der Ionenenergie, der Bestrahldauer und dem Auftreffwinkel untersucht. Je nach Aufbau des Experiments können auf diese Weise regelmäßige Wellenstrukturen oder Punktmuster erzeugt werden, was einer gezielten Aufrauung der Oberfläche entspricht, oder aber es wird eine Glättung der Oberfläche erzielt [Facs99, Fros00]. Bei der Deposition dünner Schichten auf glatten Substraten wird sehr häufig ein aufrauendes Verhalten beobachtet, das unterhalb einer kritischen Schichtdicke einem Skalengesetz folgt, bis die Rauigkeit schließlich in eine Konstante übergeht. Die Glättung einer rauen Oberfläche durch Dünnschichtdeposition wurde jedoch bisher kaum systematisch untersucht. Die Theorie sagt hier voraus, dass die Glättung einer Oberfläche frequenzabhängig abläuft und abhängig von den vorherrschenden Mechanismen unterschiedlich schnell vonstatten geht [Vill91, Mayr01]. Des Weiteren wird die minimal erreichbare Rauigkeit von der Eigenrauigkeit eines Materials beim Wachstum auf glatten Substraten bestimmt.

Daher soll im Rahmen dieser Arbeit untersucht werden, inwieweit es möglich ist, durch die Deposition unterschiedlicher Materialien eine Oberfläche zu glätten. Dafür bietet es sich an, unterschiedlichste Materialklassen wie Metalle, Oxide und Polymere zu betrachten, die eine möglichst geringe Eigenrauigkeit haben. Sind geeignete Materialien gefunden, soll die Glättung einer rauen Oberfläche systematisch im Hinblick auf die Vorhersagen der Theorie untersucht und mithilfe der Theorie simuliert werden. Durch Vergleich der Ergebnisse können so Rückschlüsse auf die Gültigkeit und Vollständigkeit der Theorie gezogen werden. Sowohl zur Analyse des frequenzabhängigen Verhaltens als auch für die Bestimmung der Glättungsmechanismen ist eine Auswertung der Oberflächenmorphologie im Fourierraum hilfreich.

Insgesamt ist die Arbeit folgendermaßen gegliedert: Nach der Vorstellung der verwendeten Herstellungs- und Untersuchungsmethoden (Kapitel 2) werden die Theorie der Glättung und die Auswertemethoden erläutert. Hierbei werden auch die am häufigsten auftretenden atomaren Mechanismen auf der Oberfläche dargestellt und im Hinblick auf die hier auftretenden Depositionsbedingungen diskutiert (Kapitel 3). In Kapitel 4 sind die verwendeten rauen Substrate beschrieben, die entweder eine gewellte Oberflächenstruktur besitzen, bei der eine räumliche Frequenz dominiert, oder aus statistisch verteilten Hügelstrukturen unterschiedlicher Größe bestehen. Kapitel 5 erläutert die Glättungseigenschaften der verschiedenen verwendeten Materialien auf den gewellten Substraten, 
1 Einleitung

vor allem im Hinblick auf ihre Effizienz. Hierbei gibt es bereits erste Hinweise auf den genauen Ablauf der Glättung und die vorherrschenden Glättungsmechanismen. Diese werden systematisch in Kapitel 6 auf statistischen Oberflächenstrukturen analysiert. Dabei kann das frequenzabhängige Glätten im Detail beobachtet und die damit verbundenen Auswirkungen der Strukturgrößen auf der Oberfläche betrachtet werden. Weiterhin werden die Glättungsmechanismen analysiert und die Ergebnisse mit der Theorie verglichen und der Glättungsverlauf simuliert. 


\section{Probenherstellung und Charakterisierung}

Im folgenden Kapitel sollen die verwendeten Herstellungs- und Untersuchungsmethoden dargestellt werden. Dazu folgt zunächst ein Abschnitt über gepulste Laserdeposition, in dem nicht nur die Dünnschichtmethode vorgestellt wird, sondern auch die für die verschiedenen Materialien verwendeten Herstellungsparameter erläutert werden. In dem Zusammenhang wird auch kurz auf die Ablationsmechanismen und die Charakteristika der deponierten Filme eingegangen. Die folgenden Unterkapitel beschreiben die verwendeten Untersuchungsmethoden.

\subsection{Gepulste Laserdeposition (PLD)}

Die gepulste Laserdeposition (pulsed laser deposition, PLD) ist eine vielseitige Dünnschichtmethode, mit der sich nicht nur Metalle, Keramiken oder Oxide deponieren lassen, sondern auch so komplexe Materialien wie Polymere. Ein Vorteil dieser Methode ist ein im Allgemeinen stöchiometrischer Materialübertrag zwischen Substrat und Target. Während der Ablation wird ein gepulster Laserstrahl hoher Intensität auf ein FestkörperTarget gelenkt. Dadurch entsteht ein Plasma, das senkrecht zur Targetoberfläche expandiert. Auf dem Substrat werden Atome mit kinetischen Energien von etwa 5-10 eV, sowie Ionen mit mittleren kinetischen Energien von etwa $100 \mathrm{eV}$ deponiert. Eine ausführliche Diskussion dieser Herstellungsmethode ist beispielsweise bei Chrisey und Hubler [Chri94] oder bei Eason [Easo07] zu finden. Eine Übersicht über die gepulste Laserdeposition von Metallen findet sich bei Krebs et al. [Kreb97, Kreb07], über die 
Ablation von Polymeren berichteten erstmals Hansen et al. [Hans88], einen guten Überblick liefert Dyer et al. [Dyer03].

Ein schematischer Aufbau der in dieser Arbeit verwendeten Anlage ist in Abbildung 2.1 skizziert. Zur Herstellung der Schichten wurde ein gepulster KrFExcimerlaser der Firma Lambda Physik vom Typ LPX 110i mit einer Wellenlänge von 248 nm und einer Pulsdauer von 30 ns verwendet. Bis auf die Polymer-Proben wurden alle Schichten bei vergleichsweise hohen Energiedichten von $4-6 \mathrm{~J} / \mathrm{cm}^{2}$ und einer Repetitionsrate von $10 \mathrm{~Hz}$ hergestellt. Für die Polymer-Schichten wurde die Energiedichte durch Verschieben der Linse auf etwa $125 \mathrm{~mJ} / \mathrm{cm}^{2}$ abgeschwächt und eine kleinere Repetitionsrate von $5 \mathrm{~Hz}$ eingestellt, um ein zu starkes Aufheizen der Targets zu vermeiden. Bei allen Schichten betrug der Target-Substrat-Abstand $50 \mathrm{~mm}$ und in der UHV-Kammer wurde ein Basisdruck von weniger als $10^{-8}$ mbar erreicht.

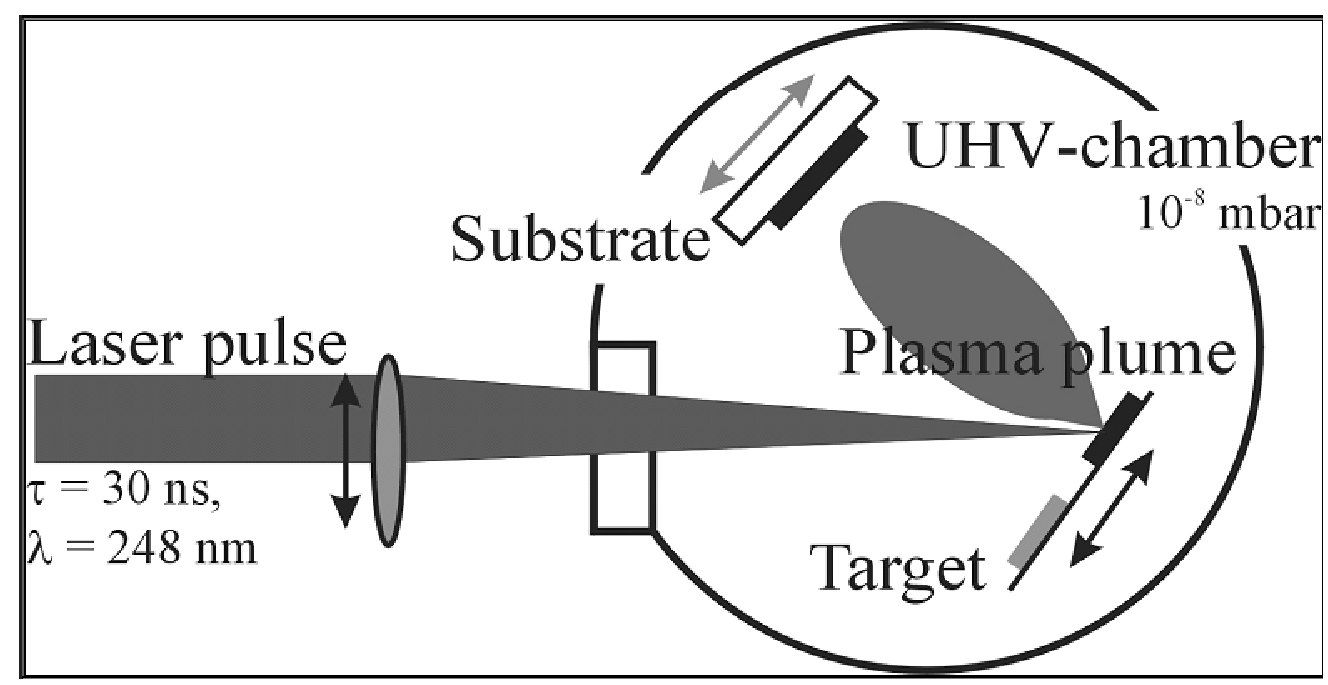

Abbildung 2.1: Schematischer Aufbau der verwendeten PLD-Kammer.

Charakteristisch für die gepulste Laserablation von Metallen, Keramiken, Oxiden und anderen Materialien ist neben der Deposition von Atomen und Ionen, die eine Schicht erzeugen, die Bildung von so genannten Tröpfchen (droplets), die durch hydrodynamisches Sputtern [Kell85] und Subsurface-Heating-Effekte [Sing90] zustande kommen. Hierbei schaukeln sich Rauigkeiten am Target durch wiederholtes Aufschmelzen und Abkühlen auf, bzw. es bilden sich Gasblasen unter der Oberfläche. Für Metalle wurde die Entstehung der Tröpfchen und die Vorgänge am Target von Fähler et al. untersucht: Es gibt zwei Arten von Tröpfchen (große und kleine), die aufgrund von Zapfenbildung am 
Target und Abriss dieser Zapfen entstehen [Fähl97]. Im Fall von Oxiden zeigen Übersichtsaufnahmen eines in dieser Arbeit verwendeten $\mathrm{ZrO}_{2}$-Targets (Abbildung 2.2 a) wellenförmige Strukturen, wie sie häufig beobachtet werden. Die durch die oben beschriebenen Effekte entstehenden Wellen besitzen in diesem Fall eine raue, grobporige Seite (Abbildung 2.2 (b)) und eine glatte Seite (Abbildung 2.2 (b), (c)). Die Unterschiede sind auf verschieden hohe Temperaturen am Target zurückzuführen. Die Risse, die auf beiden Seiten zu sehen sind, sind Spannungsrisse entlang von Korngrenzen, die dadurch entstehen, dass erhitztes Material wieder abkühlt. Das bei der gepulsten Laserdeposition entstehende Plasma wurde im Falle von $\mathrm{ZrO}_{2}$ von Voevodin et al. untersucht. Da partiell Y-stabilisiertes $\mathrm{ZrO}_{2}$ benutzt wurde (partially stabilised $\mathrm{ZrO}_{2}$ (PSZ) d.h. 3\% $\mathrm{Y}_{2} \mathrm{O}_{3}$ ), finden sich im Plasma unter Vakuumbedingungen die Atome bzw. Moleküle $\mathrm{Zr}, \mathrm{O}, \mathrm{ZrO}, \mathrm{O}_{2}$, sowie geringe Mengen von $\mathrm{Y}, \mathrm{Y}^{+1}$, YO, jeweils im angeregten Zustand [Voev00].
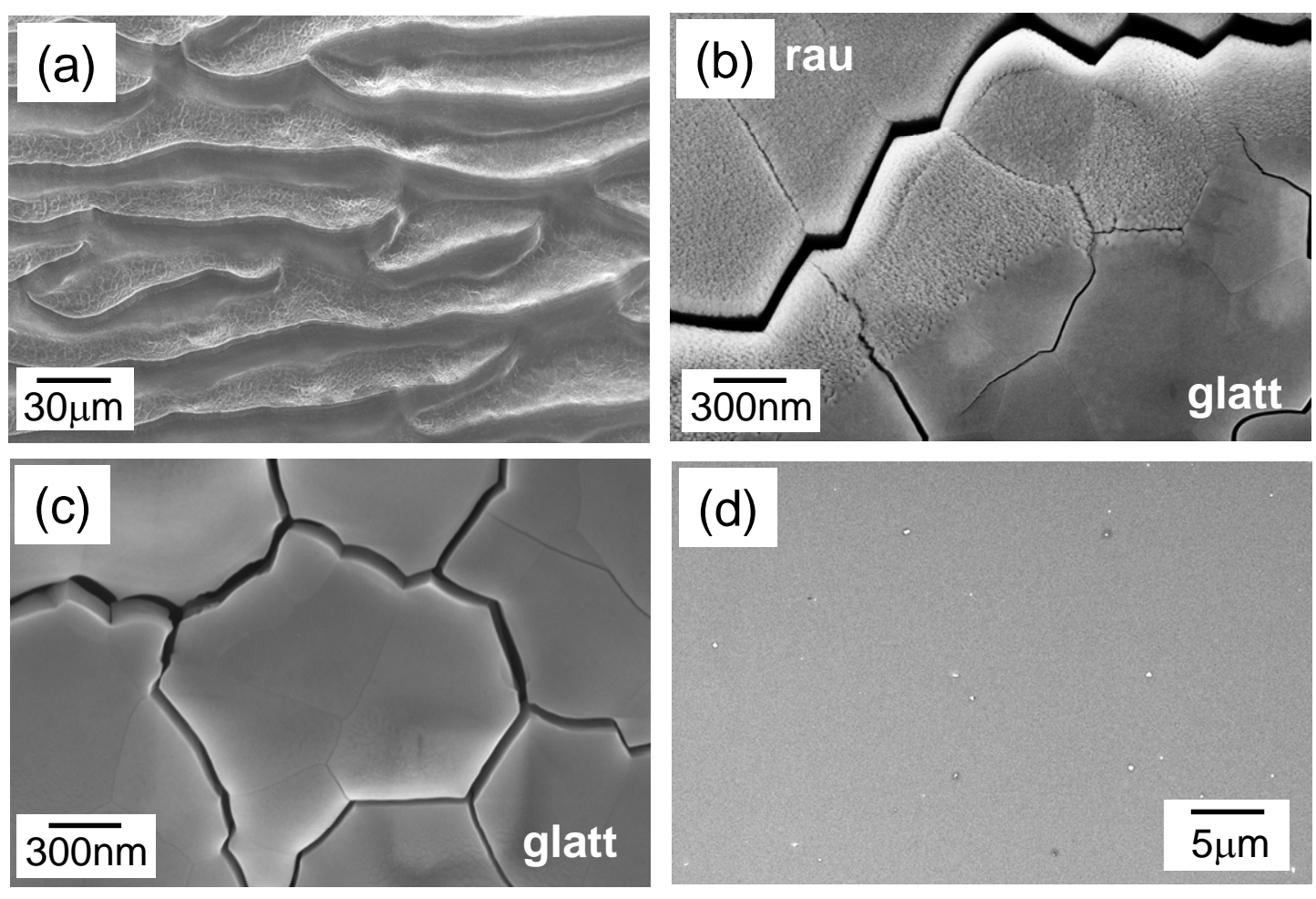

Abbildung 2.2: (a) - (c) REM-Aufnahmen eines zur Schichtherstellung verwendeten $\mathrm{ZrO}_{2}$-Targets. Der Laserstrahl hinterlässt wellenförmige Muster auf der Targetoberfläche (a), die eine raue, grobporige und eine glatte Seite haben (b). (c) zeigt die glatten Strukturen vergrößert. Die Risse sind Spannungsrisse entlang von Korngrenzen. (d) Rasterelektronenmikroskopische Übersichtsaufnahmen eines $8 \mathrm{~nm}$ dicken Films. 
Die mittels PLD bei Raumtemperatur deponierten $\mathrm{ZrO}_{2}$-Filme sind amorph [Okab05], [Voev01]. Allgemein sind $\mathrm{ZrO}_{2}$-Filme sehr hart und thermisch stabil und werden unter anderem als Verschleißschutz eingesetzt [Mill90]. Neben der Schicht scheiden sich auch einige Tröpfchen auf den Substraten ab. Eine Übersichtsaufnahme ist in Abbildung 2.2(d) dargestellt. Da die Tröpfchen nur vereinzelt auftreten und durch geeignete Methoden wie getriggerte Blenden [Barr69], off-axis Deposition in Gasathmosphäre [Holz92] oder durch sich kreuzende Plasmen (dual cross-beam-PLD [Tsel01]) vermieden werden können, werden im Folgenden nur die Rauigkeiten der Schicht selber unabhängig von den Tröpfchen untersucht.

Mittels PLD bei Raumtemperatur deponierter Kohlenstoff ist abhängig von der Ionenenergie amorph bis tetraedrisch amorph und sehr glatt [Merk99]. Der Anteil an diamantartigem, $\mathrm{sp}^{3}$-hybridisierten $\mathrm{C}$ hängt stark von der Ionenenergie im Plasma ab [Papp92]. Man erhält den höchsten $\mathrm{sp}^{3}$-Anteil von etwa 70\% bei Ionenenergien von $90 \mathrm{eV}$ [Merk98]. Im Vergleich zu $\mathrm{ZrO}_{2}$ treten bei der Ablation deutlich häufiger Tröpfchen auf, die aber ebenfalls durch die oben beschriebenen Maßnahmen vermieden werden können. Für die erfolgreiche Glättung interessiert aber auch hier nur der homogene Schichtanteil.

Die Morphologie der Poly(methyl methacrylat) (PMMA, Plexiglas®)-Schichten variiert stark je nach verwendeter Energiedichte. Bei hohen Energiedichten bilden sich am Target Tröpfchen, die durch Subsurface-Heating-Effekte [Sing90] zustande kommen und deren Entstehung im Detail von Süske et al. [Süsk06] erläutert wird. Diese können vermieden werden durch Verringerung der Energiedichte der Laserstrahlung auf etwa $150 \mathrm{~mJ} / \mathrm{cm}^{2}$ bis $160 \mathrm{~mJ} / \mathrm{cm}^{2}$ [Löse07]. Experimentell wurde das durch Verschieben der fokussierenden Linse und damit Vergrößerung des Brennflecks am Target realisiert. Infrarot (IR)-Spektren zeigen, dass die für PMMA typischen Schwingungsbanden vorhanden und die entstehenden Filme somit chemisch intakt sind [Löse06]. Im Vergleich zum Ausgangsmaterial $(1000 \mathrm{~kg} / \mathrm{mol})$ haben sie eine deutlich reduzierte mittlere Kettenlänge mit einer Molmasse von nur noch $23 \mathrm{~kg} / \mathrm{mol}$, was mittels SEC-Messungen (Größenausschlusschromatographie oder Gel-Permeations-Chromatographie, size exclusion chromatography) herausgefunden wurde [Fuch09]. Mit Rauigkeiten von nur $0,3 \mathrm{~nm}$ sind die Schichten sehr glatt. 
Die gepulste Laserdeposition von Poly-bisphenol-A-Carbonat (PC)-Filmen wurde von Faupel et al. untersucht [Faup05]: Bei geringen Energiedichten von etwa $70 \mathrm{~mJ} / \mathrm{cm}^{2}$ zeigen IR-Spektren die wichtigsten Schwingungsbanden, was auf ein mit dem Ausgangsmaterial vergleichbares Polymer schließen lässt, allerdings wieder mit verkürzter Kettenlänge und wahrscheinlich vernetzt. Bei Erhöhung der Energiedichte auf $200 \mathrm{~mJ} / \mathrm{cm}^{2}$ geht das IR-Spektrum fast vollständig verloren, die polymere Struktur der Schichten ist hier fragwürdig. Bei der Herstellung der Schichten in dieser Arbeit wurden Energiedichten von etwa $125 \mathrm{~mJ} / \mathrm{cm}^{2}$ verwendet und es ist davon auszugehen, dass das Polymer nur teilweise degradiert ist. Die Schichten selbst sind mit 0,4 nm rms-Rauigkeit relativ glatt und haften gut auf dem Substrat.

\subsection{Rasterkraftmikroskopie (AFM)}

Zur Untersuchung von Oberflächen auf der Nanometerskala eignet sich die Rastersondenmikroskopie. Die beiden gängigsten Methoden sind die Rastertunnelmikroskopie (STM), bei der der Tunnelstrom zwischen einer Spitze und einer leitenden Oberfläche gemessen wird, und die Rasterkraftmikroskopie (AFM, erstmals beschrieben von Binnig et al. [Binn86]), bei der die zwischen Probe und Spitze wirkenden Kräfte gemessen werden. Darüber hinaus gibt es viele weitere Unterarten dieser Mikroskope, bei denen beispielsweise magnetische Felder, elektrische Felder oder andere physikalische Größen detektiert werden. In dieser Arbeit wurde das AFM im Tapping Modus betrieben [Zhon93], einer Weiterentwicklung des Nicht-Kontakt Modus [Mart87], der benutzt wird, um auch weiche Proben zerstörungsfrei messen zu können. Hierbei wird der Cantilever zu Schwingungen von einigen $10 \mathrm{~nm}$ Amplitude nahe seiner Resonanzfrequenz angeregt und schwingt in einer Höhe von wenigen Nanometern so über der Probe, dass er im unteren Umkehrpunkt die Oberfläche leicht berührt, sie also „abklopft“. Durch die Wechselwirkung mit der Oberfläche ändert sich die Schwingungsamplitude. Stellt man die Regelparameter so ein, dass die Amplitude konstant gehalten wird, so misst man Profile konstanter Kraft. AFM-Bilder werden als topographisches Abbild der Oberfläche interpretiert. Neben externen Fehlerquellen wie Gebäudeschwingungen oder Schall, die 
eine Messung verfälschen können, sind vor allem auch Abbildungsfehler aufgrund stumpfer oder defekter Spitzen und thermischer Drift der Proben zu beachten. Eine Fehleranalyse ist unter anderem mit spektralen Methoden möglich [Fang97]. Den Einfluss der Spitzenform wird eingehend beschrieben bei [West94].

Alle Proben wurden an einem AFM vom Typ Nanoscope IV der Firma Veeco (ehemals Digital Instruments) untersucht. Der verwendete Scanner hatte einen maximalen Scanbereich von $12 \mu \mathrm{m} \times 12 \mu \mathrm{m}$. Jedes Bild hat eine Auflösung von $512 \times 512$ Punkten. Es wurden Si-Cantilever vom Typ BS-Tap 300 der Firma Budget Sensors benutzt, die nach Angaben des Herstellers einen Spitzenradius von $<10 \mathrm{~nm}$ haben. Zur Auswertung der AFM-Bilder wurde die mitgelieferte Software Nanoscope(R) III, Version 5.31r1 und Version 6.13r1 verwendet, mit der sich alle in der Arbeit beschriebenen Größen und Funktionen berechnen lassen.

\subsection{Transmissionselektronenmikroskopie (TEM)}

Um die Glättungsprozesse im Querschnitt untersuchen zu können, wurde die Transmissionselektronenmikroskopie benutzt. Hierfür wurde das Gerät CM 30 der Firma Philips mit maximaler Beschleunigungsspannung von $120 \mathrm{kV}$ verwendet. Anhand der TEM-Bilder konnten die inneren Grenzflächen nach der Glättung beobachtet und Strukturanalysen gemacht werden. So konnte sichergestellt werden, dass diese nicht zerstört werden. Ein TEM funktioniert im Prinzip ähnlich wie ein Lichtmikroskop, bloß dass die Probe mit hochenergetischen Elektronen durchstrahlt wird. Die Wechselwirkung der Elektronen mit dem Probenmaterial erzeugt je nach Strahlengang ein Real- oder Beugungsbild des durchstrahlten Bereichs, das mit Photoplatten aufgenommen werden kann.

Zur Präparation von hinreichend dünnen Querschnittsproben wurde ein fokussierter Ionenstrahl (focussed ion beam, FIB) des Typs Nova Nanolab 600 der Firma Fei Company verwendet. Mit diesem wurde zunächst ein Querschnitt der Probe herauspräpariert, freigeschnitten und mittels Platindeposition auf einen Halter geklebt. Die so entstandene 
Lamelle wurde auf weniger als $100 \mathrm{~nm}$ Dicke gedünnt, um sie im TEM durchstrahlen zu können.

\subsection{Rasterelektronenmikroskopie (REM)}

Um eine guten Überblick über die Morphologie von Proben und Targets zu bekommen, wurden rasterelektronenmikroskopische Bilder angefertigt. Dabei rastert ein fein gebündelter Elektronenstrahl die Probenoberfläche ab. Diese Primärelektronen wechselwirken elastisch und inelastisch mit dem Probenmaterial. Dadurch wird ein Spektrum von Elektronen unterschiedlicher Energie emittiert. Für die Bilddarstellung werden meist die Sekundär- und Rückstreuelektronen verwendet. Es wurden zwei Geräte vom Typ S360 und Supra35 der Firma Leo (ehemals Cambridge Instruments) benutzt. Die Grundzüge der Rasterelektronenmikroskopie sind beispielsweise bei Lüth beschrieben [Lüth95].

\subsection{Röntgenanalyse}

Die Röntgendiffraktometrie (XRD) eignet sich zur Strukturanalyse der deponierten dünnen Schichten. Hierbei wird die unter großen Winkeln gebeugte Strahlung in BraggBrentano Geometrie gemessen, d.h. Quelle, Probe und Detektor sind in $\theta$ / $2 \theta$ Geometrie aufgebaut. Eine ausführliche Beschreibung findet man beispielsweise bei [Cull01].

Im Falle dünner Schichten oder dünner Schichtpakete benutzt man häufig die Röntgenreflektometrie (XRR), um Informationen über die Schichtdicke der Einzel- und Doppelschichten, sowie über die Rauigkeit der Grenz- und Oberflächen zu bekommen. Dafür wird die gestreute Intensität in der oben beschriebenen Geometrie, allerdings unter sehr kleinen Einfallwinkeln aufgenommen. Da die Proben bei kleinen Winkeln überstrahlt 
werden, muss man sich Gedanken über die Spotbreite im jeweiligen Winkelbereich machen. Diese berechnet sich für das verwendete Gerät (Goniometerradius $320 \mathrm{~mm}$ ) über:

$$
\text { Spotbreite }=\frac{\left(\text { Div.schlitz }\left[{ }^{\circ}\right]\right) \cdot \pi}{180} \cdot \frac{320 \mathrm{~mm}}{\cos (90-\omega)}
$$

Bei einer Probe von $1 \mathrm{x} 1 \mathrm{~cm}^{2}$ und einem Divergenzschlitz von $1 / 32^{\circ}$, beträgt die Spotbreite bei $2 \theta=2^{\circ}\left(\right.$ d.h. $\left.\omega=1^{\circ}\right)$ somit $1 \mathrm{~cm}$, bei geringeren Winkeln ist sie größer und überstrahlt die Probe. Daher wurde bei allen Spektren die Intensität abhängig vom Strahlwinkel $\omega$ entsprechend skaliert. Die erhaltenen Spektren wurden mithilfe des IMD/XOP Softwarepakets [Wind98] angepasst. Diese beruht auf einem von Parratt entwickelten Rekursionsverfahren [Parr54]. Für alle Messungen wurde ein Philips-4-Kreisdiffraktometer vom Typ $X$ 'Pert mit einer Co-Röhre in Verbindung mit einem Fe-Filter (Wellenlänge der Co-K $\mathrm{K}_{\alpha}$-Strahlung: 0,17902 nm) verwendet.

\subsection{Schichtdickenbestimmung mittels Profilometrie und Ellipsometrie}

Zur Bestimmung der Depositionsraten der unterschiedlichen Materialien wurden in jedem Herstellungsvorgang etwa $100 \mathrm{~nm}$ dicke Schichten der verwendeten Materialien auf glatten Si-Substraten deponiert. Im Falle der metallischen und oxidischen Materialien wurde ein Teil des Substrats mit einem Klebestreifen abgedeckt. Die nach Entfernung des Klebestreifens entstandene Stufe konnte mittels Profilometrie vermessen werden. Dazu wurde ein Gerät des Typs Perthometer S8P der Firma Mahr Perthen verwendet. Bei dieser Methode wird das Höhenprofil einer Oberfläche aufgezeichnet, indem eine feine Messnadel mit geringem Druck über die Probe gezogen wird. Ihre Auslenkung in zRichtung wird registriert und als Höheninformation ausgegeben. Die Auflösung des verwendeten Gerätes liegt bei etwa 5-10 nm.

Da polymere Materialien relativ weich sind, konnten PC- und PMMA-Schichten nicht mit dem Profilometer vermessen werden. Stattdessen wurde ein Ellipsometer der 
2 Probenherstellung und Charakterisierung

Firma Nanofilm verwendet. Bei diesem Verfahren wird die Änderung des Polarisationszustandes von Laserlicht nach Reflexion an der Probe gemessen. Mithilfe eines Schichtmodells der Probe lassen sich so die Schichtdicke und der Brechungsindex bestimmen. 


\section{Theoretischer Hintergrund}

In diesem Kapitel sollen die theoretischen Grundlagen der quantitativen Analyse von Oberflächenstrukturen erläutert werden. Dazu wird zunächst auf die grundlegende Beschreibung von Rauigkeiten und die Auswertung mit Korrelationsfunktionen eingegangen (Abschnitt 3.1). Auf mesoskopischer Skala eignen sich Kontinuumsmodelle, die die Folge von atomaren Prozessen auf gröberer Skala beschreiben. Alternativ zur direkten Lösung der dabei verwendeten Gleichungen bietet sich unter gewissen Bedingungen die Fourieranalyse an, die insbesondere die Interpretation der AFM-Bilder erleichtert und eine Bestimmung der an der Oberfläche auftretenden glättenden oder aufrauenden Mechanismen möglich macht (Abschnitt 3.2). In dem Zusammenhang soll auf verschiedene aufrauende und glättende Oberflächenprozesse eingegangen werden, stets im Hinblick auf ihre Relevanz bei der hier verwendeten Dünnschichtmethode (Abschnitt 3.3). Die in der Arbeit verwendete Vorgehensweise zur Interpretation der spektralen Leistungsdichte wird in Abschnitt 3.4 erläutert. Davon ausgehend lassen sich auch Vorhersagen für die Entwicklung der Rauigkeit mit der Depositionszeit (bzw. der deponierten Schichtdicke) ableiten (Abschnitt 3.5).

\subsection{Rauigkeiten und Korrelationsfunktionen}

Die Oberflächenstruktur einer Probe wird mathematisch beschrieben, indem man jedem Ort $\vec{x}=(x, y)$ einer gedachten Bezugsebene eine Höheninformation $H(x, y)$ zuordnet. Bei Depositions- oder Abtragsprozessen mit einer mittleren Rate $F$ benutzt man 
3 Theoretischer Hintergrund

im Allgemeinen ein mitbewegtes Koordinatensystem dergestalt, dass gilt: $h(\vec{x}, t)=H(\vec{x}, t)-F t$, wobei $\mathrm{t}$ die Depositionszeit ist. Um Oberflächen miteinander vergleichen zu können, braucht man quantitative Größen, die die Oberflächenbeschaffenheit charakterisieren. Dazu eignet sich erstmal die Rauigkeit, die im Falle der meist verwendeten rms-Rauigkeit (root-mean-square roughness) folgendermaßen definiert ist [Bara95]:

$$
\sigma_{r m s}=\sqrt{\left\langle h^{2}(\vec{x})\right\rangle_{\vec{x}}}
$$

Dieser Wert allein ist nicht ausreichend, da er keinerlei Informationen über die lateralen Strukturgrößen, wie z.B. Hügel-, Insel- oder Korngrößen auf der Oberfläche enthält. Um Oberflächen detaillierter zu beschreiben, eignen sich Korrelationsfunktionen im Realraum oder im Fourierraum.

Im Fourierraum wird häufig die zweidimensionale spektrale Leistungsdichte (power spectral density, PSD) verwandt, die sich folgendermaßen berechnet:

$$
\operatorname{PSD}(\vec{k})=|F T(h(\vec{x}))|^{2}
$$

wobei $k_{x}$ und $k_{y}$ räumliche Frequenzen sind und FT die Fouriertransformation bezeichnet. $\operatorname{PSD}(\vec{k})$ hat die Einheit (Länge) ${ }^{4}$ und stellt ein Maß dafür da, welche Oberflächenfrequenzen wie stark zur Oberflächenrauigkeit beitragen. Gewöhnlich normalisiert man diese Funktion noch durch azimuthale Mittelung:

$$
\operatorname{PSD}(k)=\left\langle|F T(h(\vec{x}))|^{2}\right\rangle_{|\vec{k}|=k}
$$

Eine genauere Analyse der spektralen Leistungsdichte und Hinweise zu ihrer Interpretation finden sich in Kapitel 3.4. Im Falle isotroper Oberflächen hängt $\operatorname{PSD}(\vec{k})$ nur noch vom Betrag $k=|\vec{k}|=\left(k_{x}^{2}+k_{y}^{2}\right)^{1 / 2}$ ab. Dann gilt für die rms-Rauigkeit [Bara95]:

$$
\sigma_{r m s}^{2}(t)=2 \pi \int_{0}^{\infty} k P S D(k) d k=2 \pi \int_{0}^{\infty} k|\hat{h}(k, t)|^{2} d k
$$


Bei der Interpretation von AFM-Daten sowohl im Real- als auch im Fourierraum ist zu beachten, dass der sinnvoll interpretierbare räumliche Frequenzbereich abhängig ist von der Größe des Scanbereichs $L_{\text {scan }}$ und dem Spitzenradius $R_{\text {tip }}$. Als Faustregel gilt [Fang97]:

$$
\frac{10}{L_{\text {scan }}} \leq k \leq \frac{1}{R_{\text {tip }}}
$$

Allgemein ist zu beachten, dass das mittels AFM gemessene Oberflächenprofil stets eine Faltung aus der Form der Spitze und der tatsächlichen Oberfläche darstellt. Für die Auswertung der oben eingeführten Funktionen muss man annehmen, dass die Spitze keine starken Artefakte aufweist, d.h. gleichmäßig geformt ist und ihr Radius klein im Vergleich zu den Strukturgrößen auf der Probe.

\subsection{Kontinuumsmodell}

Für die Modellierung von Oberflächenprozessen ist die Art und Weise entscheidend, wie sich unter den gegebenen Bedingungen (z.B. Wachstum oder Abtrag einer Schicht) eine gegebene Oberfläche mit der Zeit entwickelt. Ein sehr erfolgreiches Werkzeug für das Verständnis des Verhaltens eines Systems bei verschiedenen Wachstumsprozessen sind stochastische Differentialgleichungen. Sie beschreiben eine Grenzfläche typischerweise auf mesoskopischen Längenskalen, die Details auf atomaren Skalen werden vernachlässigt. Ganz allgemein gilt für die Entwicklung der Höhe mit der Zeit:

$$
\frac{\partial H(\vec{x}, t)}{\partial t}=G[H(\vec{x}, t)]+F+\eta(\vec{x}, t)
$$

bzw. der relativen Höhe im mitbewegten Koordinatensystem:

$$
\frac{\partial h(\vec{x}, t)}{\partial t}=G[h(\vec{x}, t)]+\eta(\vec{x}, t)
$$

Das Funktional $G[h(\vec{x}, t)]$ beschreibt hierbei mathematisch die physikalischen Prozesse an der Oberfläche und stellt die Summe aus den umgebungsabhängigen Veränderungen 
dar. Mögliche Beiträge ergeben sich aus der Höhenfunktion $h$ selbst und deren räumlichen Ableitungen. $\eta(\vec{x}, t)$ ist ein stochastischer Rauschterm, der räumlich und zeitlich unkorreliert ist (sog. weißes Rauschen) und berücksichtigt, dass Prozesse wie die Teilchendeposition immer zufälliger Natur sind. Es gilt:

$$
\begin{gathered}
\langle\eta(\vec{x}, t)\rangle=0 \\
\left\langle\eta(\vec{x}, t) \eta\left(\vec{x}^{\prime}, t^{\prime}\right)\right\rangle=2 R \delta^{2}\left(\vec{x}-\vec{x}^{\prime}\right) \delta\left(t-t^{\prime}\right)
\end{gathered}
$$

$R$ wird als Rauschstärke bezeichnet und $\delta$ ist die mathematische Deltafunktion. Im Fall von unkorreliertem Rauschen ist $R$ konstant.

Häufig ist es nicht möglich oder sehr schwierig, Evolutionsgleichungen vom Typ 3.7 direkt zu lösen. Eine Alternative ist die Lösung der Gleichung im Fourierraum. Dies ist insbesondere dann sinnvoll, wenn das Funktional G, das die Prozesse auf der Oberfläche beschreibt, im Ortsraum linear in $h$ ist. Ein solcher linearer Ansatz wird im Folgenden immer benutzt. Berücksichtigt man räumliche Ableitungen verschiedener Ordnung, so lautet er allgemein [Tong94]:

$$
\frac{\partial \hat{h}(\vec{k}, t)}{\partial t}=-\sum_{i} b_{i} k^{i} \hat{h}(\vec{k}, t)+\hat{\eta}(\vec{k}, t), \quad i \in \mathbb{N}
$$

Hierbei sind $b_{i}$ Konstanten, $\hat{h}(\vec{k}, t)$ und $\hat{\eta}(\vec{k}, t)$ sind die Fouriertransformierten der jeweiligen Funktionen. Frühere Arbeiten haben gezeigt, dass den Termen für $i=1 \ldots 4$ verschiedene Transportprozesse wie z.B. Diffusion, viskoses Fließen, Sputtererosion etc. zugeordnet werden können [Herr50, Mull57]. Dies soll im Unterkapitel 3.3 ausführlich erläutert werden. Gleichung 3.10 wird im deterministischen Fall, also ohne einen Rauschterm, gelöst durch die Exponentialfunktion:

$$
\hat{h}(\vec{k}, t)=\hat{h}(\vec{k}, t=0) \cdot \exp \left(-t \sum_{i} b_{i} k^{i}\right)
$$

$\hat{h}(\vec{k}, t=0)$ ist die anfängliche Höhenfunktion. Hierbei entscheiden die Vorzeichen der Koeffizienten $b_{i}$ darüber, ob der zugehörige Term zur Aufrauung $\left(b_{i}<0\right)$ oder zur Glättung $\left(b_{i}>0\right)$ beiträgt. Um den relativen Beitrag verschiedener Prozesse zu analysieren, muss man die Lösungsfunktion näher betrachten. Es zeigt sich, dass für jeden glättenden Prozess der Ordnung $i$ die charakteristische Abklingzeit (die Zeit, nach der die Amplitude der 
ursprünglichen Oberflächenstruktur auf 1/e abgefallen ist) gegeben ist durch $\left(b_{i} k^{i}\right)^{-1}$. Ein Maß für die Stärke eines Prozesses ist das Inverse der Abklingzeit, also $\left(b_{i} k^{i}\right)$. Somit lässt sich das relative Gewicht zweier glättender Prozesse der Ordnungen $l, m$ durch Betrachtung des folgenden Quotienten bestimmen:

$$
b_{m} k^{m} / b_{l} k^{l}
$$

Die Übergangsfrequenz liegt dort, wo der Quotient den Wert 1 hat. Bis zu der zur Übergangsfrequenz gehörenden Wellenlänge dominiert ein Prozess der Ordnung $l$, ab der Wellenlänge ist hauptsächlich der Prozess der Ordnung $m$ für die Strukturbildung verantwortlich.

\subsection{Oberflächenprozesse bei der Schichtdeposition mit PLD}

Im Rahmen dieser Arbeit werden sämtliche dünne Schichten mittels PLD hergestellt. Wie bereits in Kapitel 2.1 erläutert, treffen hierbei sowohl Atome mit einer Energie von einigen eV (5-10 eV), als auch Ionen, deren kinetische Energie mit etwa $100 \mathrm{eV}$ höher ist, auf dem Substrat auf. Neben den „klassischen“ Prozessen wie z.B. Diffusion beeinflussen auch diese relativ hohen kinetischen Energien die vorherrschenden physikalischen Transportphänomene am Substrat, die für Glättung oder Aufrauung verantwortlich sind. Alle diese Prozesse zeigen ein charakteristisches Skalenverhalten, das beschreibt, um wie viel schneller kleinere Strukturen eine (geometrische) Veränderung durchlaufen im Vergleich zu größeren Strukturen [Herr50]. Das äußert sich mathematisch in der Frequenzabhängigkeit $k^{i}$ der einzelnen Terme der Funktion 3.10. Im Folgenden sollen die unterschiedlichen Prozesse sortiert nach ihrer Ordnung $i$ erläutert und ihre Relevanz für die hier hergestellten Schichten diskutiert werden.

Der wichtigste Transportprozess der Ordnung $i=1$ ist viskoses Volumenfließen [Mull59, Orch62]. Hierbei berücksichtigt man, dass energiereiche Ionen die Schicht lokal 
viskos deformieren können. Ist die Dicke dieser aufgeschmolzenen Bereiche („thermal spikes“) sehr viel größer als die typische Strukturgröße an der Oberfläche, so spricht man von Volumenfließen. Die Hauptkomponente der Teilchenbewegung liegt hier parallel zur Substratnormalen (im Gegensatz zum Oberflächenfließen, was später erläutert wird) [Orch62]. Die für das lokale Aufschmelzen notwendigen Energien liegen im MeV-Bereich [Mayr01]. Da die Ionen bei PLD nur maximal einige 100eV haben, kann dieser Prozess als irrelevant ausgeschlossen werden.

Es gibt viele unterschiedliche Mechanismen, die der Ordnung $i=2$ zuzuordnen sind. Da wären Desorption [Herr50, Mull57], Sputtererosion [Brad88], Abschattungseffekte [Sevc73] und ballistischer Transport [Mose05]. Desorption bezeichnet das thermische Wiederabdampfen von Adatomen. Unter Abschattungseffekten versteht man das Überwachsen von Tälern, ohne dass diese komplett ausgefüllt werden. Dadurch entsteht eine poröse Schicht. Ursache hierfür können sehr geringe Diffusion oder ein kleiner Auftreffwinkel der Teilchen auf dem Substrat sein. Beide Mechanismen sind besonders bei thermischen Energien (etwa 0,1 eV) relevant und sind daher aufgrund der höheren Energien, die bei PLD auftreten, als unbedeutend anzusehen. Sputtererosion bezeichnet das Herausschlagen von Oberflächenatomen in Folge von Stoßkaskaden, die von implantierten Atomen oder Ionen mit Energien von einigen $\mathrm{eV}$ ausgelöst werden. Abhängig von Parametern wie dem Auftreffwinkel oder der Ionenenergie kann dieser Prozess sowohl glättend als auch aufrauend wirken [Zibe08]. Ballistischer Transport bezeichnet einen Prozess, der relativ effektiv zur Glättung rauer Oberflächen beitragen kann. Trifft ein Ion parallel zur lokalen Oberflächennormalen auf dem Substrat auf, so hat die ausgelöste Stoßkaskade keine Vorzugsrichtung entlang der Oberflächenkontur. Bei der Schichtdeposition auf einer rauen Oberfläche stimmen allerdings die lokale Oberflächennormale und die Einfallsrichtung der Ionen nicht mehr zwingend überein. Ein Ion, das auf einer Hügelflanke auftrifft, löst eine Kaskade aus, die die Oberflächenatome bevorzugt ins Tal schiebt. MD-Simulationen zeigen, dass dieser Mechanismus speziell bei sehr niedrigen Ionenenergien $(\leq 100 \mathrm{eV})$ und kovalent gebundenen Systemen relevant ist [Mose05], was den Energien entspricht, die insbesondere auch bei PLD auftreten.

Die Volumendiffusion ist ein Transportmechanismus der Ordnung $i=3$ [Herr50, Mull57] und kann für die hier verwendeten Materialien ebenfalls vernachlässigt werden, da 
für Festkörper Diffusion im Volumen wesentlich unwahrscheinlicher ist als Diffusion an der Oberfläche.

Oberflächendiffusion ist ein Prozess der Ordnung i=4 [Herr50, Mull57], ebenso wie viskoses Oberflächenfließen. Unter Oberflächenfließen versteht man analog zum Volumenfließen das lokale Aufschmelzen der Oberfläche bei Bestrahlung mit Ionen mit dem Unterschied, dass hier die Energie der Teilchen nur ausreicht, eine sehr dünne Schicht an der Oberfläche zu verflüssigen. Die Hauptkomponente der Teilchenbewegung ist hier parallel zur Substratnormalen [Orch62]. Oberflächenfließen ist einer der vorherrschenden Mechanismen bei Ionenenergien im keV-Bereich [Vaut08]. Da die bei PLD auftretenden Energien deutlich geringer sind, ist anzunehmen, dass dieser Prozess im Vergleich zu der sehr viel wichtigeren Oberflächendiffusion nur eine untergeordnete Rolle spielt.

Die bisher erläuterten Prozesse sind in vielen Fällen ausreichend, um das Verhalten von amorphen Materialien zu beschreiben. Beim Wachstum kristalliner Schichten ist die Beschreibung der Oberfläche deutlich schwieriger, da dreidimensionales Inselwachstum und Koaleszenzphänomene beobachtet werden. Hierbei führt die Energieminimierung von kristallinen Strukturen zur Ausbildung von Stufen oder Korngrenzen und damit zu zusätzlichen Rauigkeiten, die in den Modellen bisher nicht berücksichtigt wurden. Ihre mathematische Betrachtung ist äußerst schwierig. Häufig erhält man Exponenten $i$, die deutlich größer sind als 4 [Erle97, Eise01], also einen Abfall der Spektren, der deutlich steiler ist als -4 .

\subsection{Frequenzabhängigkeit der Glättung}

Im Folgenden sollen die Veränderungen der spektralen Leistungsdichte während eines Glättungsprozesses betrachtet werden. Für die vorliegende Arbeit ist dabei nicht ihre Abhängigkeit von der Depositionszeit t, sondern von der Schichtdicke d interessant, da die direkt gemessen wird. Es gilt allgemein: $t=F^{-1} \cdot d$, wobei $F$ die Depositionsrate, also eine Konstante ist. Im Falle des in Kapitel 3.2 gewählten linearen Ansatzes lässt sich die spektrale Leistungsdichte folgendermaßen berechnen [Vill91]: 


$$
\operatorname{PSD}(k, d)=R(k) \frac{1-\exp \left(-2 \cdot F^{-1} \cdot d \sum b_{i} k^{i}\right)}{\sum b_{i} k^{i}}
$$

Hierbei ist $R(k)$ die Fouriertransformierte der Rauschstärke, die bereits in Gleichung 3.9 verwandt wurde. Im Falle von zeitlich und räumlich unkorreliertem Rauschen, ist $R$ eine Konstante. Gleichung 3.13 gilt für den Fall, dass die Ausgangsoberfläche glatt war und beschreibt, dass beim Schichtwachstum durch den Rauschenterm $R$ Rauigkeiten erzeugt werden, die bereits während der Deposition durch verschiedene Prozesse der Ordnung $i$ wieder ausgeglättet werden können. Dies geschieht abhängig von $k^{i}$. Die graphische Darstellung von Gleichung 3.13 in Abhängigkeit der deponierten Schichtdicke (Abbildung 3.1 (a)) zeigt, dass die Glättungsmechanismen zunächst im Bereich hoher Frequenzen, d.h. kleiner Strukturen auf der Oberfläche angreifen, was zu einem Abfallen der spektralen Leistungsdichte in diesem Bereich führt. Die Steigung dieses Abfalls ist hierbei ein Hinweis auf die vorherrschenden Mechanismen. In Abbildung 3.1 wurde der Einfachheit halber nur ein Mechanismus der Ordnung 4 (Diffusion) zugelassen. Dort, wo noch keine Glättungsmechanismen angegriffen haben, ist ein Plateau, das durch den Beitrag der Rauschstärke / des weißen Rauschens entsteht [Liu02]. Es verschiebt sich mit steigender Schichtdicke $d$ (bzw. $t=F^{-1} \cdot d$ ) zu höheren Leistungsdichten und kleineren Frequenzen.
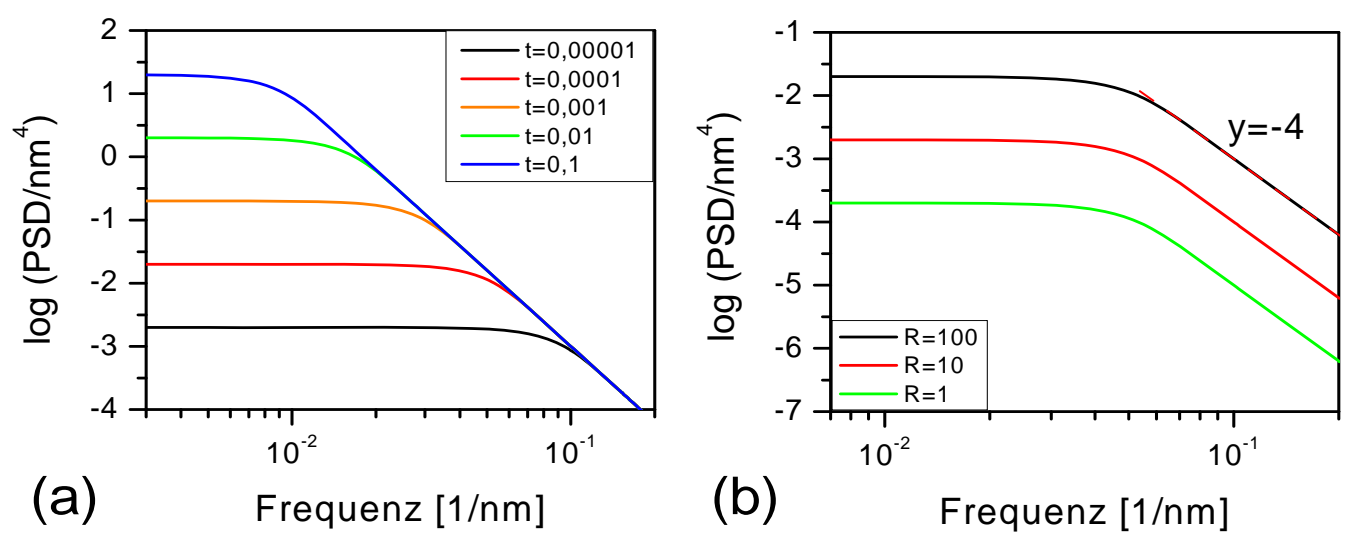

Abbildung 3.1: (a) Veränderung der spektralen Leistungsdichte mit der Schichtdicke $d$ (bzw. $t=F^{-1} \cdot d$ ) in beliebigen Einheiten. (b) Die spektrale Leistungsdichte in Abhängigkeit der Rauschstärke $R$. In beiden Fällen wurde nur ein Glättungsmechanismus der Ordnung 4 (Diffusion) angenommen. 
Die Übergangsfrequenz zwischen dem Plateau und dem Bereich linearen Abfallens ist eine charakteristische Größe, die aufgrund der begrenzten Reichweite der Glättungsmechanismen entsteht. Man findet die dazugehörige Wellenlänge auf der Oberfläche als dominierende Strukturgröße, wie Inselgröße oder im Fall kristalliner Materialien als Korngröße wieder. Abbildung 3.1(b) verdeutlicht die Auswirkungen des Rauschens bei konstant gehaltener Schichtdicke $d$. Je größer das Rauschen ist, desto höher liegen die Spektren und desto größer ist somit die Gesamtrauigkeit der Oberfläche (vergleiche Gleichung 3.13 und 3.4).

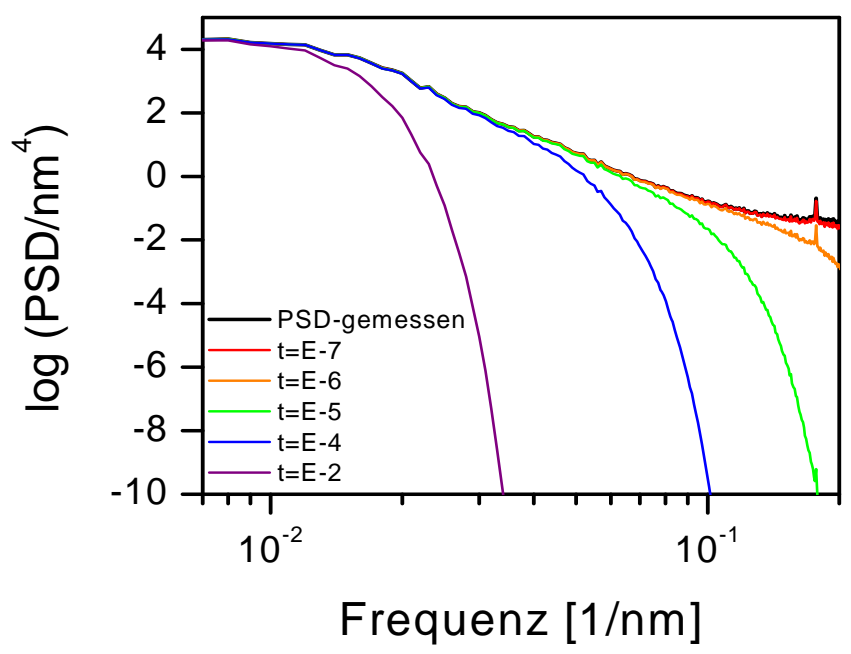

Abbildung 3.2: Veränderung der spektralen Leistungsdichte aufgrund der exponentiellen Glättung einer rauen Ag-Oberfläche (erster Summand aus Gleichung 3.14). Das Ursprungsspektrum (schwarz) ist das mit AFM gemessene Spektrum einer Ag-Schicht. Als Glättungsmechanismus wurde nur Diffusion (Ordnung 4) zugelassen.

Ist die Anfangsoberfläche rau, so muss ein zusätzlicher additiver Term in Gleichung 3.13 berücksichtigt werden, der die sukzessive Glättung der anfänglichen Strukturen beschreibt [Mayr01]:

$$
P S D(k, d)=P S D\left(k, d_{0}\right) \cdot \exp \left(-2 \cdot F^{-1} d \sum b_{i} k^{i}\right)+R(k) \frac{1-\exp \left(-2 \cdot F^{-1} d \sum b_{i} k^{i}\right)}{\sum b_{i} k^{i}}
$$

$\operatorname{PSD}\left(k, d_{0}\right)$ ist die anfängliche spektrale Leistungsdichte der rauen Oberfläche, die mit steigender deponierter Schichtdicke $d$ exponentiell geglättet wird. Hierbei spielen 
dieselben Mechanismen eine Rolle wie beim bisher diskutierten Schichtwachstum und führen erneut zur frequenzabhängigen Veränderung der Oberfläche: Bei kleinen Schichtdicken werden zunächst die hohen Frequenzen gegen Null gehen, mit steigender Schichtdicke auch tiefere. Der exponentielle Abfall der ursprünglichen spektralen Leistungsdichte (also nur der erste Summand aus Gleichung 3.14) ist beispielhaft in Abbildung 3.2 dargestellt. Als Ausgangsspektrum wurde das mittels AFM gemessene Spektrum von Ag gewählt, als Glättungsterm wieder ausschließlich Diffusion (i=4) zugelassen. Man erkennt deutlich, dass es eine Grenzfrequenz gibt, oberhalb der mit der Glättung begonnen wird. Diese Grenzfrequenz verschiebt sich mit zunehmender Depositionszeit zu kleineren Frequenzen hin.
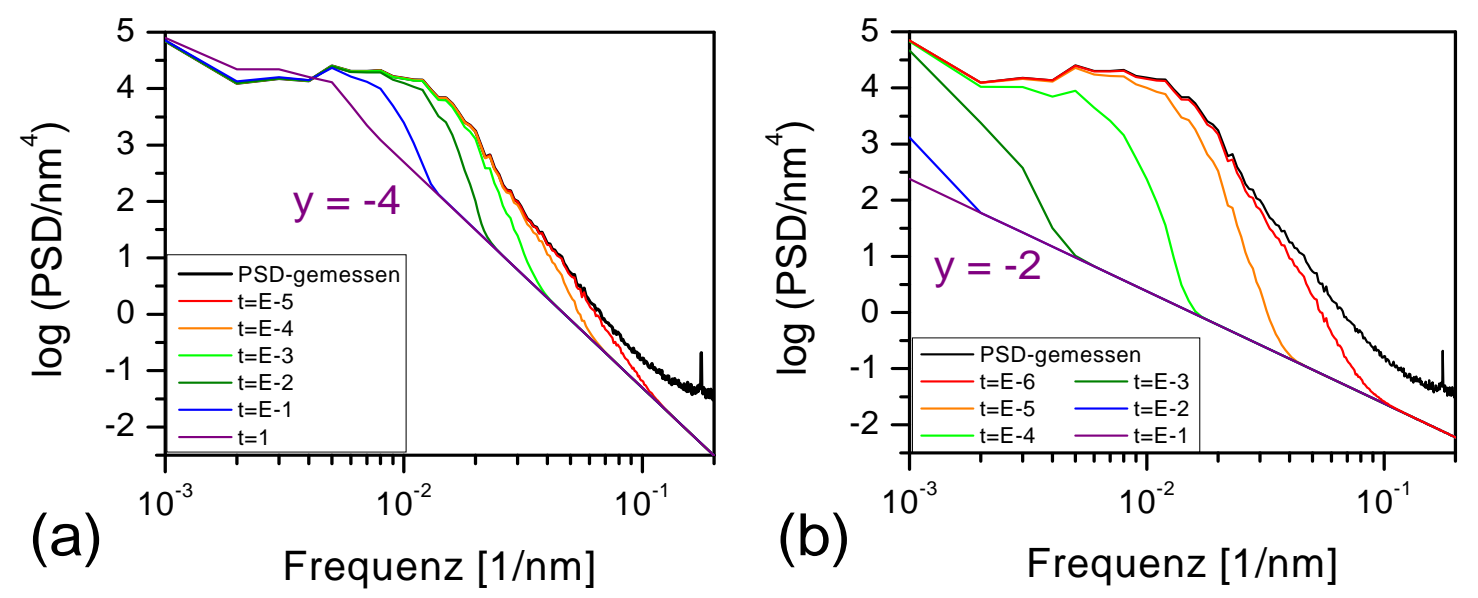

\begin{abstract}
Abbildung 3.3: Veränderung der spektralen Leistungsdichte einer rauen Schicht im Laufe der Glättung (graphische Darstellung von Gleichung 3.14). In (a) ist beispielhaft nur Diffusion (Ordnung 4), in (b) nur ballistisches Glätten bzw. Sputtererosion (Ordnung 2) als Glättungsmechanismus zugelassen.
\end{abstract}

Im Experiment treten nun beide Terme auf, da der exponentielle Abfall der ursprünglichen spektralen Leistungsdichte (erster Term) überlagert wird vom Anwachsen der durch die Schichtdeposition bedingten spektralen Leistungsdichte der neuen Schicht (zweiter Term, bzw. Gleichung 3.13). Graphisch ist dieser Vorgang in Abbildung 3.3 dargestellt, in (a) für den Fall, dass nur ein Glättungsmechanismus der Ordnung 4 (Diffusion) existiert, in (b) nur für einen Mechanismus der Ordnung 2 (Ballistisches Glätten / Sputtererosion). Als Ausgangsspektrum wurde wieder die Leistungsdichte einer realen Ag-Schicht gewählt. Zusammenfassend zeigen diese Überlegungen, dass die 
Glättung einer Oberfläche frequenzabhängig ist. Mit zunehmender Schichtdicke $d$ wird die ursprüngliche spektrale Leistungsdichte nach und nach bei immer kleineren Frequenzen reduziert. Dieser Abfall ist exponentiell und wird überlagert von der spektralen Leistungsdichte der aufwachsenden Schicht. Dabei legt die Ordnung $i$ der angreifenden Mechanismen die Steigung des Endspektrums fest und die Größe der Rauschstärke $R$ beeinflusst die Höhe der spektralen Leistungsdichte. Demnach muss ein geeignetes Glättungsmaterial zwei Anforderungen erfüllen: Es muss eine möglichst geringe intrinsische Rauigkeit (oder Eigenrauigkeit) beim Wachstum auf glatten Substraten haben und es muss schnell glätten, das heißt die von der Oberfläche vorgegebenen Rauigkeiten schnell eliminieren.
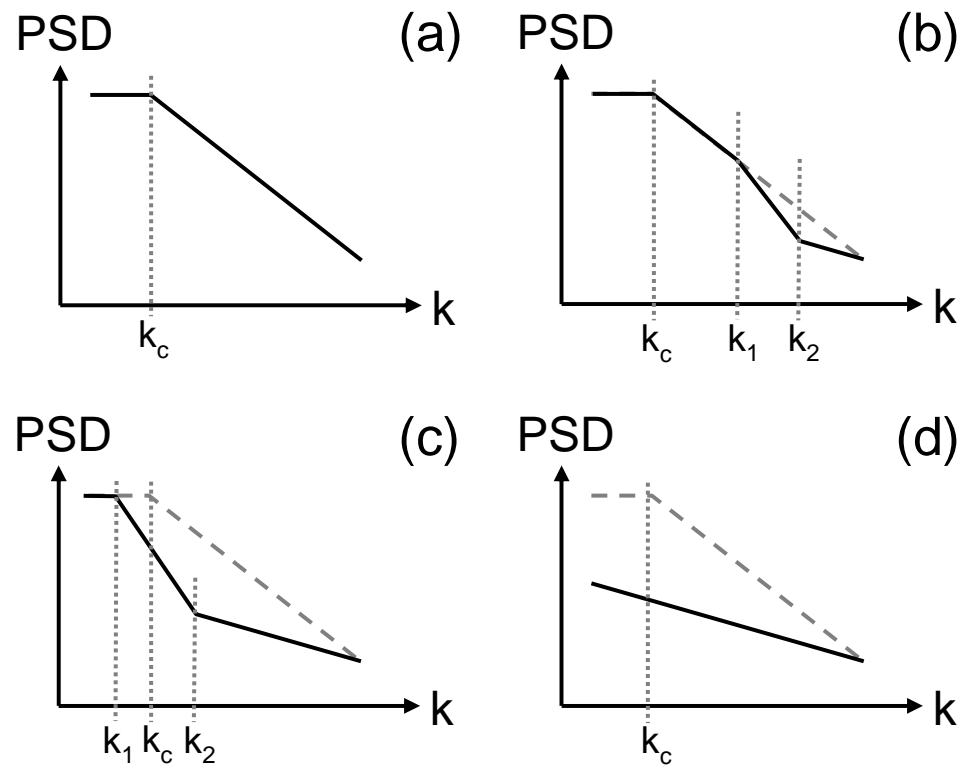

\begin{abstract}
Abbildung 3.4: Veränderung der spektralen Leistungsdichte der ungeglätteten Oberfläche (a) mit der mittleren Inselgröße $1 / k_{c}$ während eines erfolgreichen Glättungsprozesses. (b) und (c) zeigen zwei Stadien des Glättungsprozesses. Oberhalb der Frequenz $k_{2}$ sind die Frequenzen bereits komplett ausgeglättet, unterhalb von $k_{1}$ hat der Glättungsprozess noch nicht begonnen. In (d) wurde der gemessene Frequenzbereich komplett ausgeglättet. Die Graphen sind in doppeltlogarithmischer Auftragung dargestellt.
\end{abstract}

Es wird anhand der graphischen Darstellungen deutlich, dass während der Glättung verschiedene Abknickpunkte in der spektralen Leistungsdichte zu erwarten sind. Dies ist in Abbildung 3.3 deutlich zu erkennen und zum besseren Verständnis schematisch in 
Abbildung 3.4 dargestellt: Die ungeglättete Oberfläche (a) mit einer dominierenden Strukturgröße $\lambda_{c}=1 / k_{c}$ (hier entspricht sie dem mittleren lateralen Inseldurchmesser) wird mit zunehmender Schichtdicke sukzessive geglättet und zwar stets in dem Frequenzbereich zwischen $k_{1}$ und $k_{2}$ (b). Oberhalb von $k_{2}$ ist die Oberfläche bereits komplett geglättet, das Spektrum kann hier durch die Gleichung 3.13 dargestellt werden. Die Frequenz $k_{2}$ entspricht also der größten komplett ausgeglätteten Struktur der Oberfläche. Unterhalb von $k_{1}$ sind die Auswirkungen der Glättungsmechanismen so gering, dass das System nach wie vor das Spektrum der ursprünglichen Oberfläche aufweist.

Der Frequenzbereich, in dem die Glättung stattfindet, verschiebt sich mit zunehmender Schichtdicke $d$ zu größeren Frequenzen hin, bis schließlich die Anfangsrauigkeiten vollständig ausgeglättet sind. Es bleibt das Spektrum übrig, das dem Wachstumsverhalten auf einer glatten Oberfläche bei großen Schichtdicken (stationärer Grenzfall $t \rightarrow \infty$ ) entspricht (Abbildung 3.4(d)). In diesem stationären Grenzfall kann man Gleichung 3.14 vereinfachen zu [Mayr01]:

$$
\lim _{d \rightarrow \infty} P S D(k, d)=\frac{R(k)}{\sum b_{i} k^{i}}
$$

Dieser Grenzfall wird wie beschrieben für große $k$-Werte früher erreicht als für kleine, d.h. kleine Strukturen auf der Oberfläche werden schneller ausgeglättet als große. Damit lassen sich experimentell bestimmte spektrale Leistungsdichten in linearer Näherung einfach interpretieren, sofern ein Transportprozess der Ordnung $i$ dominiert: In diesem Fall fällt die spektrale Leistungsdichte proportional $\mathrm{zu} k^{-i}$ ab, das heißt in doppeltlogarithmischer Auftragung erhält man einen linearen Abfall der Steigung $-i$.

\subsection{Entwicklung der Rauigkeit}

Berechnet man die spektrale Leistungsdichte, so analysiert man die Oberfläche zu einem festen Zeitpunkt $t$ und wertet die räumlichen Informationen aus. Im letzten Kapitel wurde dann die Entwicklung der spektralen Leistungsdichte $\operatorname{PSD}(k, t)$ in Abhängigkeit von der Depositionszeit (Schichtdicke) untersucht. Im Folgenden soll die zeitliche 
Entwicklung der Rauigkeit betrachtet werden, die zu jedem Zeitpunkt jeweils eine räumlich gemittelte Größe darstellt. Die Rauigkeit berechnet sich aus (Kapitel 3.1, Gleichung 3.4):

$$
\sigma_{r m s}^{2}(t)=2 \pi \int_{0}^{\infty} k P S D(k) d k
$$

Setzt man hier die spektrale Leistungsdichte aus Gleichung 3.14 ein, ist es sinnvoll für die anfängliche Oberfläche $P S D\left(k, t_{0}\right)$ zwei Grenzfälle zu betrachten, um zu analytischen Lösungen zu kommen [Vaut07]. Hierbei wird der stochastische Beitrag vernachlässigt und die Lösung des linearen Modells (Gleichung 3.11) verwendet:

1) Die spektrale Leistungsdichte der ursprünglichen Oberfläche besteht nur aus einer Hauptfrequenz $k_{s}$, das bedeutet, die reale Oberfläche wird von der entsprechenden Wellenlänge dominiert. Dies ist in sehr grober Näherung bei den verwendeten Substratoberflächen mit Wellenstrukturen der Fall, die in Kapitel 4 beschrieben werden. Es gilt $\operatorname{PSD}\left(k, t_{0}\right)=\delta\left(k-k_{s}\right)$. Man erhält für die Überlagerung von verschiedenen Prozessen der Ordnung $i$ :

$$
\sigma(t) \propto \exp (- \text { const } \cdot t)
$$

Die Rauigkeit nimmt also unabhängig von der Ordnung eines oder mehrerer glättender Prozesse exponentiell ab, wobei sich die Konstante berechnet aus const $=2 \sum b_{i} k^{i}$.

2) Die spektrale Leistungsdichte der ursprünglichen Oberfläche ist unabhängig vom Wellenvektor, das heißt auf der realen Oberfläche sind alle Frequenzen gleichermaßen vertreten. Dies tritt auf, wenn die Strukturbildung beim Schichtwachstum allein von unkorreliertem Rauschen verursacht wird. Für jeden Prozess der Ordnung $i \geq 1$ erhält man [Vaut07]:

$$
\sigma(t) \propto t^{-1 / i}
$$


3 Theoretischer Hintergrund

Insgesamt hat dieses Kapitel gezeigt, dass ein Glättungsprozess, der durch Schichtwachstum erzeugt wird, stets eine Überlagerung zweier Prozesse ist: Einerseits wird die ursprüngliche Oberfläche mit zunehmender Schichtdicke ausgeglättet, andererseits wird eine intrinsische Rauigkeit aufgrund des Schichtwachstums erzeugt, die nicht von der Substratmorphologie, sondern vom Wachstumsverhalten auf glatten Substraten desselben Materials abhängt. Diese Eigenrauigkeit entspricht der Endrauigkeit, die minimal erreicht werden kann. Für mit PLD hergestellte Schichten sind vor allem drei atomare Transportmechanismen relevant: Diffusion, Sputtererosion und ballistisches Glätten. Die Rauigkeit nimmt abhängig von der Beschaffenheit der rauen Oberfläche exponentiell oder mit einem Potenzgesetz ab. 


\section{Analyse der Substrate}

Um in den folgenden Kapiteln das Glättungsverhalten verschiedener Materialien untersuchen zu können, müssen zunächst die zu glättenden Unterlagen charakterisiert werden. Hierbei wird nicht nur die rms-Rauigkeit analysiert, sondern allgemein der Aufbau der Oberfläche mit ihren durch die Herstellungsmethode bedingten charakteristischen Strukturgrößen, die sich im Fourierraum gut darstellen lassen, und ihre Homogenität. Im weiteren Verlauf der Arbeit soll unterschieden werden zwischen statistisch rauen Substraten, die isotrop sind und bei denen keine Oberflächenfrequenzen besonders ausgezeichnet sind (z.B. durch Inselwachstum hergestellt), und strukturiert rauen Substraten, die sowohl anisotrop sein können als auch charakteristische Oberflächenfrequenzen aufweisen können (z.B. Wellenstrukturen, Punktstrukturen). Konkret wird in Kapitel 4.1 auf die Eigenschaften von mittels Sputtererosion strukturiertem (100)Silizium eingegangen. Des Weiteren wird das Wachstumsverhalten von Ag auf Si untersucht (Abschnitt 4.2), da sich auf diese Weise in guter Näherung statistisch raue Oberflächen erzeugen lassen.

\subsection{Strukturiertes Si}

Für die Glättungsexperimente wurden (100)Si-Wafer durch Ionenbeschuss aufgeraut. Die Präparation wurde von B. Ziberi und F. Frost am Leibniz-Institut für Oberflächenmodifizierung e.V. in Leipzig durchgeführt. Die verwendeten $\mathrm{Ar}^{+}$-Ionen hatten Energien von $1200 \mathrm{eV}$ und einen Einfallswinkel von $15^{\circ}$ zur Oberflächennormalen. Die 
Stromdichte betrug $1,87 \cdot 10^{15}$ Ionen/ $\left(\mathrm{cm}^{2} \mathrm{~s}\right)$, die Bestrahlzeit etwa $2 \mathrm{~h}$. AFMUntersuchungen an verschiedenen Positionen auf den Wafern haben gezeigt, dass durch den Bestrahlungsprozess periodische Oberflächenwelligkeiten entstanden sind, deren Strukturen auf dem einzelnen Wafer sehr homogen sind, allerdings von Wafer zu Wafer unterschiedlich hoch sind. Abbildung 4.1 zeigt zwei Beispiele unterschiedlicher Ausschnittsgröße.
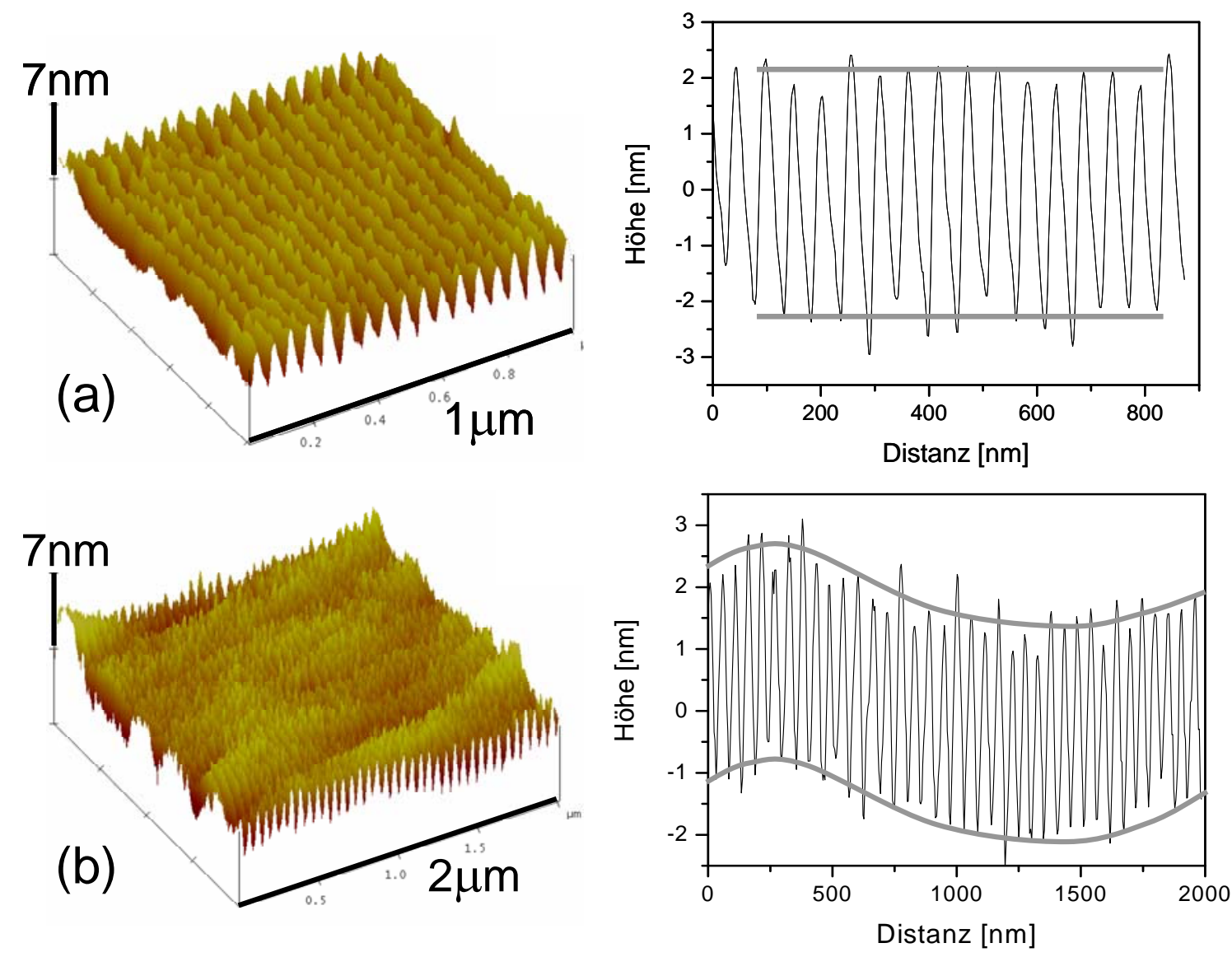

\begin{abstract}
Abbildung 4.1: AFM-Bilder unterschiedlicher Größe zweier durch Ionenbeschuss strukturierter (100)-Si Wafer, jeweils als 3D-Bild (links) und ein repräsentatives Höhenprofil (rechts). Die Höhe der Welligkeiten schwankt leicht von Wafer zu Wafer.
\end{abstract}

Die Periode der Strukturen beträgt etwa $(55 \pm 5) \mathrm{nm}$, die durchschnittliche Höhe von Wafer 1 beträgt $(4,5 \pm 0,5) \mathrm{nm}$, von Wafer $2(3,5 \pm 0,5) \mathrm{nm}$. Dies entspricht rmsRauigkeiten von etwa 1,3 nm bzw. 1,1 nm. Neben der für die Glättung interessanten Wellenstruktur existiert eine weitere langwelligere Überstruktur, die in etwa senkrecht zu 
den kurzwelligeren Strukturen steht und besonderes auf größeren Bildausschnitten gut erkennbar ist (Abbildung 4.1(b)). Sie hat eine Wellenlänge von etwa (430 \pm 50 ) nm und ist unterschiedlich stark ausgeprägt. Für die weiteren Untersuchungen kann sie vernachlässigt werden, da sie lediglich einen Rauigkeitsbeitrag bei sehr niedrigen Frequenzen liefert, der in den meisten Fällen nicht mehr gemessen wird.

Die rasterelektronenmikroskopische Übersichtsaufnahme der Wellenstruktur in Abbildung 4.2 (links) zeigt, dass die einzelnen Wellen nicht absolut parallel angeordnet sind, sondern sich gelegentlich verzweigen oder ineinander übergehen. Das hat Einfluss auf die Periodizität, die daher mit relativ großem Fehler behaftet ist.
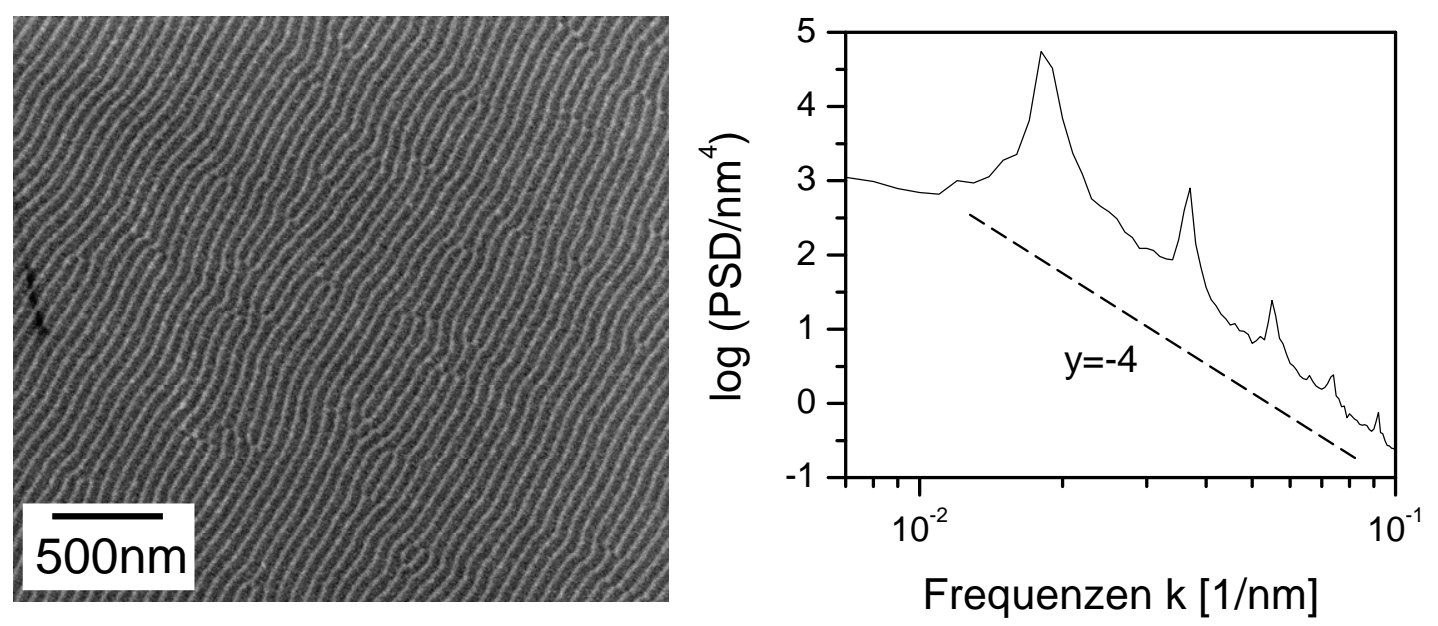

Abbildung 4.2: Links: Rasterelektronenmikroskopische Übersichtsaufnahme der Wellenstrukturen auf der Si-Oberfläche. Es lassen sich Verzweigungen erkennen, die die Periodizität beeinflussen. Rechts: Spektrale Leistungsdichte berechnet aus einem AFM-Bild der Größe 1x1 $\mu \mathrm{m}^{2}$.

Abbildung 4.2 (rechts) zeigt die spektrale Leistungsdichte eines $1 \mathrm{x} 1 \mu \mathrm{m}^{2}$ großen Bildausschnittes. Der Abfall zu höheren Frequenzen hin ist Folge der Frequenzabhängigkeit der einzelnen Transportprozesse an der Oberfläche. Überlagert wird der Abfall durch ein Hauptmaximum bei einer Frequenz von etwa 1/(55 nm) und höheren Ordnungen, die Vielfachen der Grundfrequenz entsprechen. Diese spiegeln die periodische Struktur auf der Oberfläche wieder. Die Halbwertsbreite des Hauptmaximums gibt an, wie stark fehlerbehaftet die Periodizität der Wellenstruktur ist, und ist daher ein Maß für die Ordnung der Strukturen. Beachtet man die logarithmische Auftragung, so wird klar, dass 
das Hauptmaximum den größten Beitrag zur Rauigkeit leistet (vergleiche Formel 3.16). Somit ist eine effektive Abnahme des Rauigkeitswertes erst dann zu erwarten, wenn die dominante Hauptfrequenz ausgeglättet wird. Man erwartet des Weiteren in grober Näherung einen exponentiellen Abfall der rms-Rauigkeit (Gleichung 3.17).

\subsection{Statistische Oberflächenstrukturen}

Bisher wurden Oberflächen vorgestellt, bei denen bestimmte räumliche Frequenzen in Form von periodischen Strukturen auf der Oberfläche dominieren. Im Folgenden soll die gezielte Herstellung rauer Oberflächen betrachtet werden, bei denen es keine Vorzugsrichtung gibt, d.h. die Rauigkeit ist charakterisiert durch eine statistische Anordnung von unterschiedlich großen Strukturen auf der Oberfläche. In der Theorie ist dies möglich über das Modell der ballistischen Deposition [Bara95], bei der ein deponiertes Teilchen an dem Platz liegen bleibt, an dem es auftritt. Praktisch ist es schwierig solche Bedingungen einzustellen, da immer Relaxationsmechanismen auftreten, die zur Glättung oder Strukturbildung führen. Im Folgenden soll das Inselwachstum von Ag im Hinblick auf die Rauigkeitsentwicklung untersucht werden, um eine möglichst gute Unterlage für Glättungsexperimente zu finden.

Abbildung 4.3 zeigt AFM-Bilder einiger unterschiedlich dicker Ag-Filme auf glatten Si-Substraten, Abbildung 4.4 Höhenprofile ausgewählter Proben. Da Ag-Atome auf einer Si-Oberfläche sehr weit diffundieren können, bilden sich bereits im Anfangsstadium des Wachstums relativ große Inseln von 20-25 nm Breite und 2-5 nm Höhe. Mit zunehmender Schichtdicke wachsen die Inseln durch Koaleszenzprozesse sowohl in der Höhe als auch in der Breite, bis bei 4-5 nm Schichtdicke der Perkolationsübergang erreicht ist. Bei einer Schichtdicke von etwa 8-10 nm ist die Schicht geschlossen. Danach werden bei anhaltender Materialdeposition verbliebene Täler und Unebenheiten der Oberfläche ausgeglättet (man vergleiche die Höhenprofile einer $11 \mathrm{~nm}$ und einer $22 \mathrm{~nm}$ Schicht) und es findet dreidimensionales Säulenwachstum der einzelnen Körner statt. Hierbei werden teilweise Körner überwachsen, was zu einer Vergrößerung des mittleren Korndurchmessers führt. 


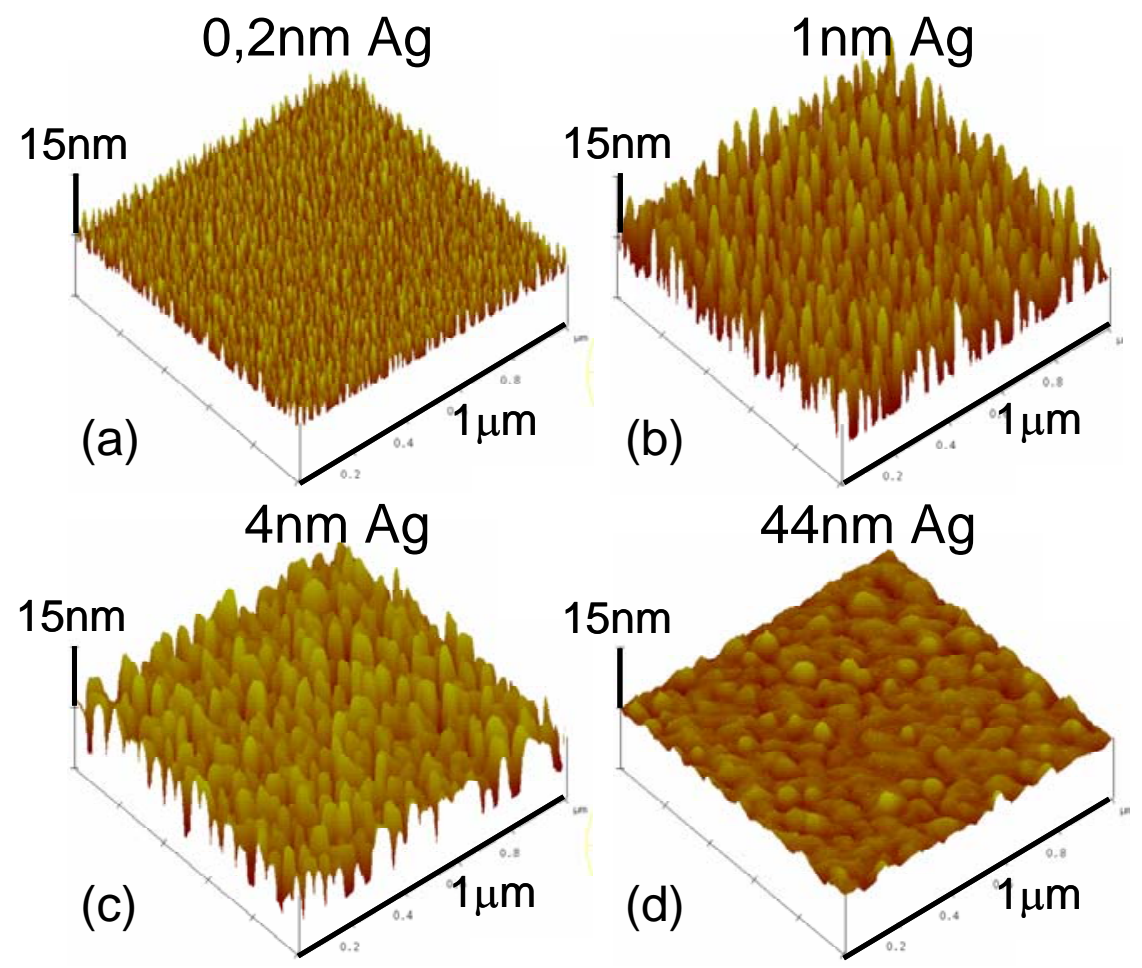

Abbildung 4.3: Die AFM-Bilder zeigen die Mikrostruktur von unterschiedlich dicken Ag-Schichten auf polierten Si-Substraten. Während die Schichten (a) und (b) noch einzelne Inseln unterschiedlicher Größe zeigen, ist Schicht (c) bereits kurz vor dem Perkolationsübergang. Schicht (d) ist geschlossen.

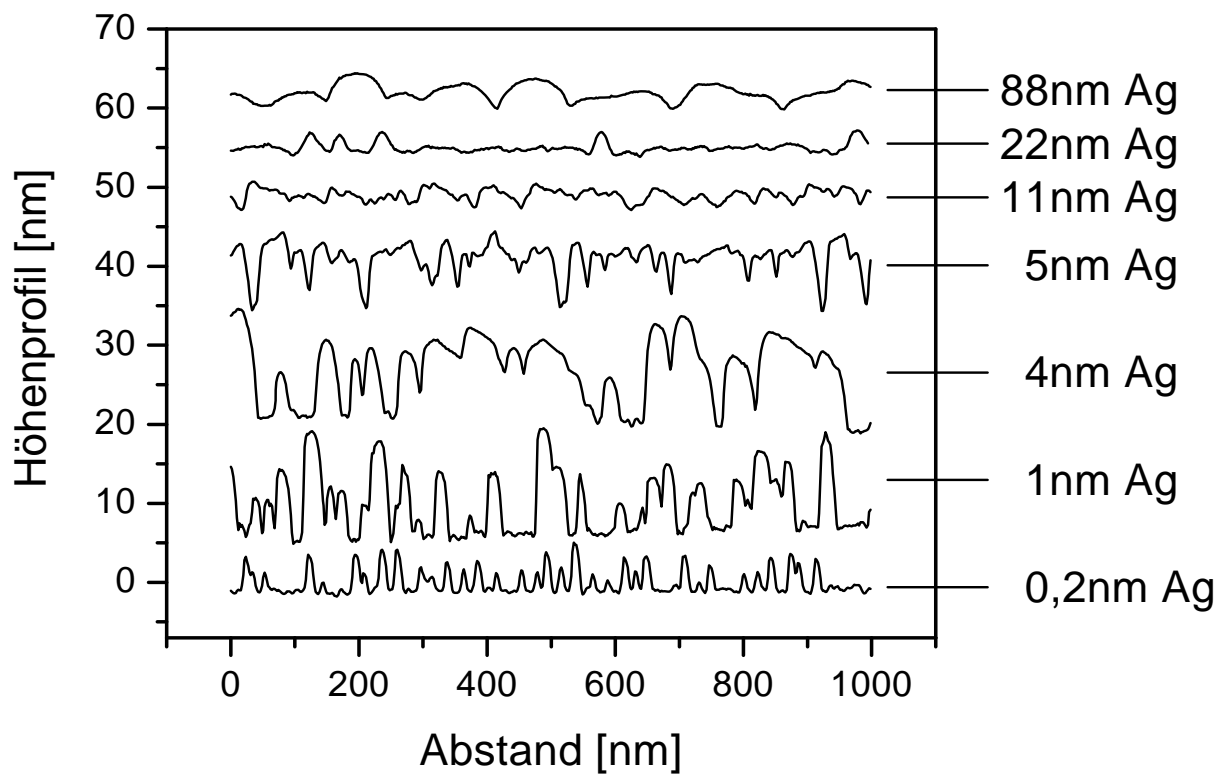

Abbildung 4.4: Zum besseren Vergleich übereinander angeordnete Höhenprofile unterschiedlich dicker Ag-Schichten auf glatten Si-Substraten. 
Das beschriebene Wachstumsverhalten wirkt sich so aus (Abbildung 4.5), dass zunächst die rms-Rauigkeit aufgrund des ausgeprägten Inselwachstums auf Werte von bis zu $4 \mathrm{~nm}$ ansteigt und dann wieder abnimmt, sobald verstärkt Koaleszenzphänomene auftreten (insbesondere unvollständige Koaleszenz, vergleiche [Röde05]). Sie erreicht ihr Minimum von etwa 0,8 nm, wenn die Schichten komplett geschlossen sind (bei etwa 8$10 \mathrm{~nm}$ Schichtdicke). Ursache für die Glättung der Oberflächenstrukturen sind hier Zipping-Mechanismen [Nix99, Hoff76], die bewirken, dass spitze Winkel zwischen zwei Körnern glattgezogen und gemeinsame Korngrenzen gebildet werden. Danach nimmt die Rauigkeit wieder $\mathrm{zu}$, da die durch das Überwachsen entstehenden langwelligeren Strukturen von den Relaxationsmechanismen nicht ausreichend geglättet werden.

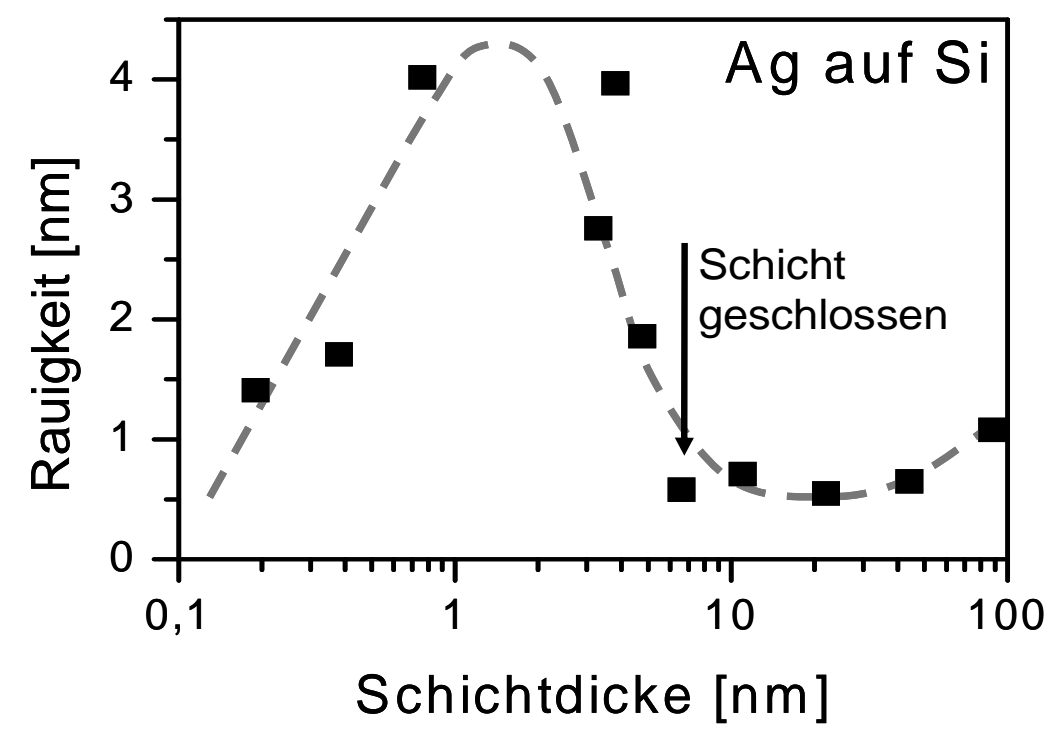

Abbildung 4.5: Rms-Rauigkeiten der Ag-Schichten aufgetragen gegen die deponierte Schichtdicke. Die Rauigkeit nimmt zunächst aufgrund von Inselwachstum zu, dann durch Koaleszenzprozesse ab und erreicht ihr Minimum, wenn die Schichten erstmals komplett geschlossen sind.

Der Vergleich der spektralen Leistungsdichten (Abbildung 4.6 (a)) der Schichten zeigt zunächst, dass die Spektren einen ähnlichen Verlauf besitzen wie die Rauigkeit: Zunächst nimmt die Gesamthöhe mit der Ag-Schichtdicke zu und dann ab $1 \mathrm{~nm}$ Schichtdicke wieder ab. Der Grund hierfür ist, dass die Rauigkeit das Integral über das Gesamtspektrum multipliziert mit der Frequenz ist (vergleiche Formel 3.4). Des Weiteren 
zeigen alle Graphen einen Abknickpunkt, der sich mit zunehmender Schichtdicke zu niedrigeren Frequenzen verschiebt. Dieser Abknickfrequenz lässt sich eine Korrelationslänge $k_{c}$ zuordnen, die in Abbildung 4.6(b) gegen die Schichtdicke aufgetragen ist. Sie entspricht dem typischen lateralen Inseldurchmesser auf der Oberfläche und kann im Fall von Ag der kristallinen Korngröße zugeordnet werden. Sie nimmt wie erwartet mit steigender Schichtdicke zu und es gilt $\lambda_{c}(d)=1 / k_{c}(d) \sim d^{0,25}$. In der Literatur werden im Fall von Kornwachstum Exponenten zwischen 0,25 und 0,5 diskutiert [Eise01, Ande84]. Zweidimensionales Kornwachstum liefert Exponenten von etwa 0,4. Exponenten von 0,25 wie hier bei Ag sagen Thijssen et al. voraus für den Fall von dreidimensionalem Kristallitwachstum im Anfangsstadium des Schichtwachstums und späterem säulenartigen Wachstum, wobei eine dreidimensionale Oberfläche erhalten bleibt [Thij92]. Das passt hervorragend zu den hier vorliegenden Ergebnissen (vergleiche Abbildung 4.4).
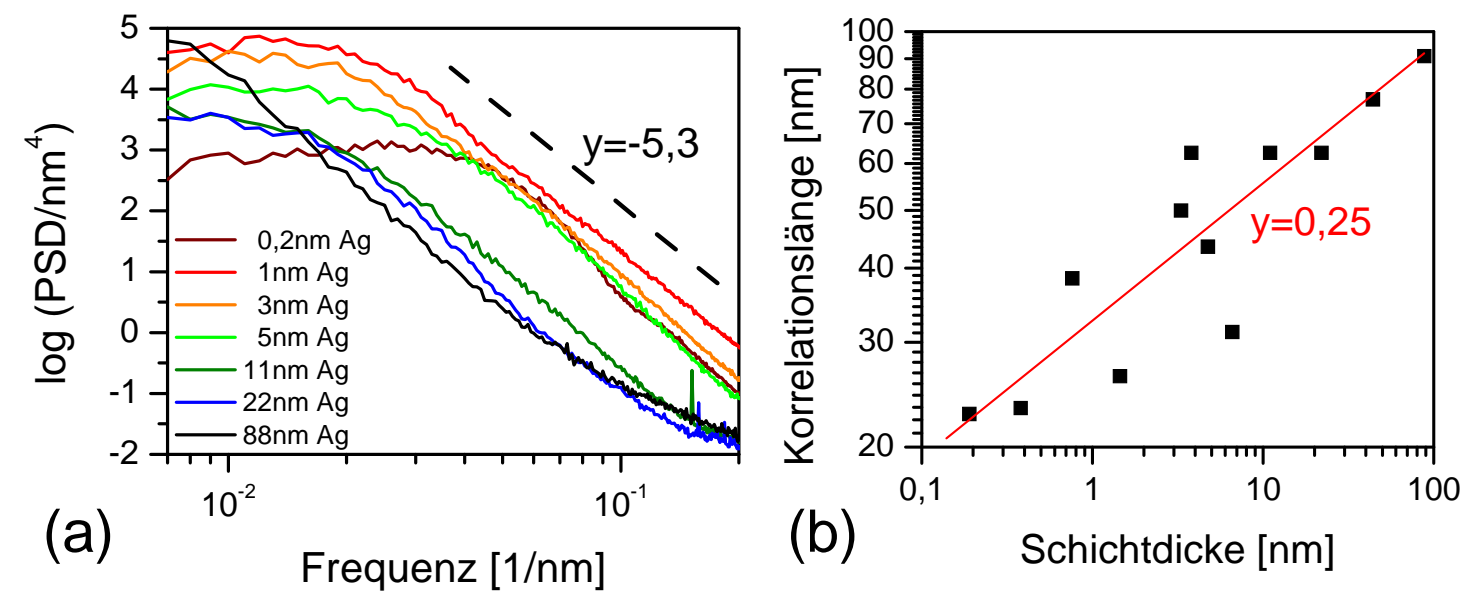

\begin{abstract}
Abbildung 4.6: (a) Veränderung der spektralen Leistungsdichte der Oberflächenstrukturen mit der Ag-Schichtdicke. (b) Korrelationslängen der Schichten aufgetragen gegen die deponierte Schichtdicke. Die Gerade ist an die Daten gefittet und zeigt grob den Verlauf.
\end{abstract}

Betrachtet man den Abfall der spektralen Leistungsdichte, so zeigen alle Graphen im Bereich hoher Frequenzen einen Abfall, der deutlich steiler ist als bei amorphen Oberflächen, die minimal eine Steigung von -4 haben. Dies wurde bereits in Kapitel 3.3 diskutiert und ist auf das kristalline Wachstum zurückzuführen. 
4 Analyse der Substrate

In Kapitel 6 werden die hier beschriebenen Ag-Schichten unterschiedlicher Dicke als raue Unterlage für Glättungsexperimente verwendet. Dabei sollen unter anderem die auftretenden Mechanismen und die Vorgänge bei der Glättung detailliert untersucht und die Auswirkungen der unterschiedlich großen mittleren Strukturgrößen auf dem Substrat analysiert werden. Zuvor werden in Kapitel 5 verschiedene Materialien im Hinblick darauf untersucht, ob sie sich als Glättungsmaterial eignen. 


\section{Materialspezifische Glättung welliger Oberflächenstrukturen}

Um eine Oberfläche effizient durch Deposition einer neuen Schicht zu glätten, ist die Wahl des Glättungsmaterials von entscheidender Bedeutung. Ziel ist es dabei, mit möglichst geringen Schichtdicken eine möglichst vollständige Glättung zu erreichen. Die theoretischen Analysen in Kapitel 3.4 haben gezeigt, dass ein geeignetes Material dafür eine möglichst geringe Eigenrauigkeit auf glatten Substraten haben muss und die auf dem Substrat vorhandenen Rauigkeiten schnell ausgeglättet werden müssen. Des Weiteren sollte das gewählte Material eine stabile Schicht bilden und im Idealfall speziell gewünschte Eigenschaften haben, also je nach technischer Anforderung z.B. hart oder weich, leitend oder nichtleitend etc. sein. Im Folgenden sollen die Möglichkeiten untersucht werden, die verschiedene Materialien und Materialklassen in dieser Hinsicht bieten. Als Beispiel für Metalle und kristalline Materialien wird das Glättungsverhalten von Ag betrachtet (Abschnitt 5.1), das in Form von kristallinen Inseln wächst (vergleiche Kapitel 4.2). C ist ein vergleichsweise einfaches amorphes System, das in der Literatur kaum aufrauendes Verhalten auf glatten Substraten zeigt [Merk99]. Die Glättung mit C wird in Unterkapitel 5.2 analysiert. Als Vertreter polymerer Materialien werden PMMA (Abschnitt 5.3) und PC (Abschnitt 5.4) untersucht. Vor allem PMMA entnetzt auf SiSubstraten und zeigt damit ein ganz eigenes Glättungsverhalten. Schließlich soll die Glättung durch Oxide am Beispiel von $\mathrm{ZrO}_{2}$ betrachtet werden (Abschnitt 5.5). Sämtliche Materialien werden in Abschnitt 5.6 miteinander verglichen und ihre Glättungseffizienz in Abhängigkeit von ihren Eigenschaften diskutiert. 
Als Substrat dient für alle Experimente das mit Ionenstrahl strukturierte (100)Silizium, wie es bereits in Kapitel 4.1 beschrieben wurde. Die Wellen haben eine Periodizität von $(55 \pm 5) \mathrm{nm}$ und eine rms-Rauigkeit von etwa 1,3 nm. Auf diese Weise kann die Ausglättung dieser dominanten Frequenz betrachtet und für verschiedene Materialien verglichen werden.

\subsection{Glättung durch Ag}

Ag ist ein kristallines Metall, das aufgrund hoher Diffusion ein sehr ausgeprägtes Inselwachstum auf glatten Si-Wafern zeigt, was zu einer starken Aufrauung der Oberfläche in den Anfangsstadien des Wachstums führt (vergleiche Kapitel 4.2). Bei dickeren Schichten wird die Rauigkeit durch Koaleszenz und Island-Zipping-Prozesse wieder reduziert und eine minimale Rauigkeit von $0,8 \mathrm{~nm}$ tritt bei gerade geschlossenen Schichten auf. Die Korngrößen des Ag variieren zwischen $20 \mathrm{~nm}$ und $100 \mathrm{~nm}$ abhängig von der deponierten Schichtdicke. Bei geschlossenen Schichten beobachtet man Säulenwachstum und damit eine vergleichsweise raue Oberfläche mit rms-Werten von 0,8 nm und mehr (Kapitel 4.2). Im Folgenden soll untersucht werden, ob sich das Wachstumsverhalten auf gewellten Oberflächen verändert und inwiefern ein Inselwachstum mit Korngrößen ähnlich der Wellenperiodizität von 55 nm zur Oberflächenglättung beitragen kann.

In Abbildung 5.1 sind rasterkraftmikroskopische Bilder der Wellenstrukturen nach Deposition der angegebenen Mengen von Ag dargestellt. Bei dünnen Filmen ist Ag noch sehr feinkörnig verteilt, die Wellen sind weiterhin gut erkennbar. Mit zunehmender Schichtdicke werden die Körner größer und überdecken die Wellen. Bereits nach der Deposition von $8 \mathrm{~nm} \mathrm{Ag}$ ist die Strukturierung des Substrats nicht mehr erkennbar. Mit wachsender Schichtdicke nimmt die Korngröße zu, ähnlich wie es beim Wachstum auf glatten Substraten beobachtet wurde. Die Höhenprofile, die zur besseren Gegenüberstellung übereinander geschichtet dargestellt sind (Abbildung 5.2), ermöglichen den direkten Vergleich der Oberflächen. Sie zeigen das beschriebene Verhalten, wobei hier bereits ab $3 \mathrm{~nm}$ Schichtdicke die Wellenstrukturen verschwunden zu sein scheinen, während sie in den kompletten AFM-Bildern noch zu erahnen sind. 


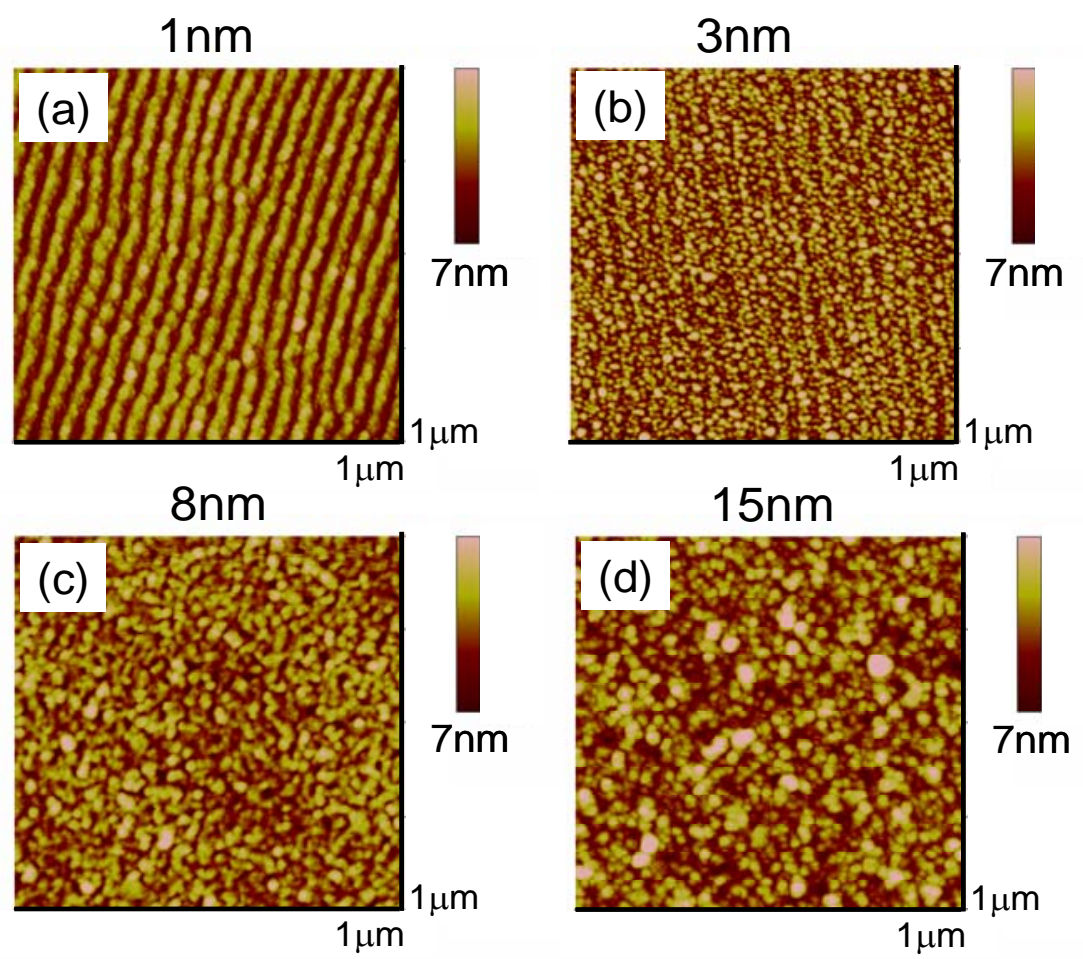

Abbildung 5.1: Rasterkraftmikroskopische Bilder der Wellensubstrate nach der Deposition von Ag. Die Schichtdicken sind jeweils angegeben. Mit zunehmender Schichtdicke überdecken die kristallinen Körner die Struktur des Substrates.

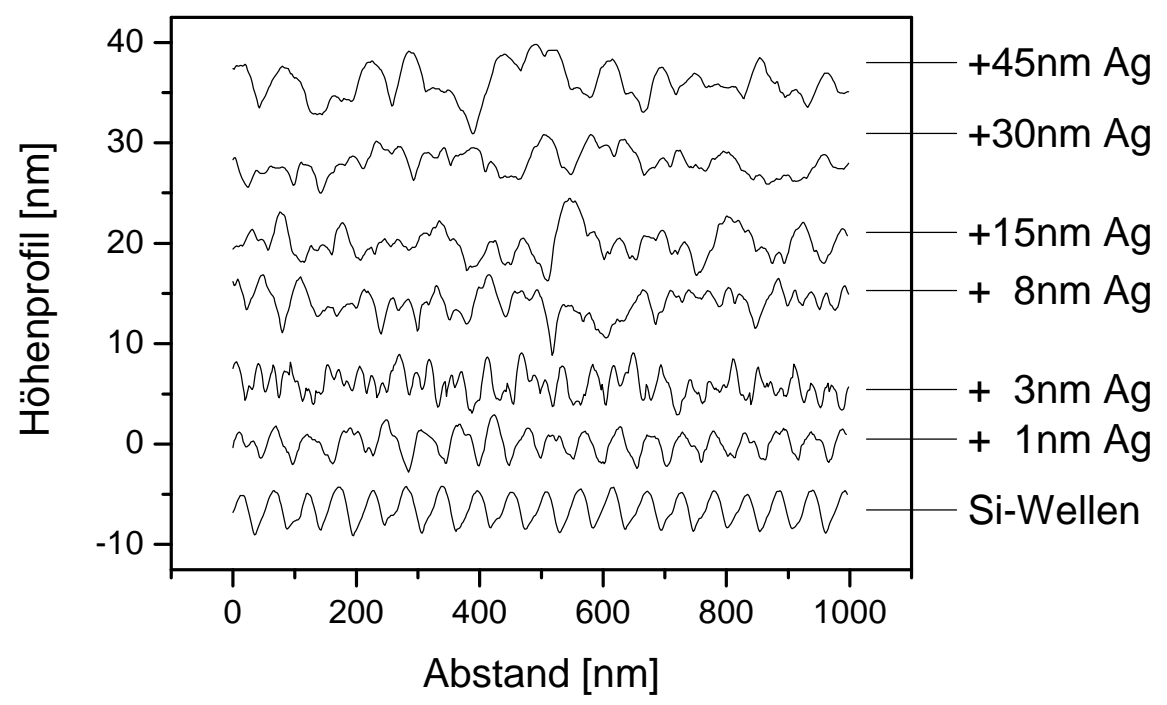

Abbildung 5.2: Übereinander angeordnete Höhenprofile der mit unterschiedlicher Menge Ag bedeckten Si-Wellenstrukturen. Die ursprüngliche Morphologie wird schnell überlagert von der intrinsischen Rauigkeit des Ag, die durch das starke Kornwachstum entsteht und auch auf glatten Substraten zu beobachten ist. 
Die Entwicklung der Rauigkeiten mit der Ag-Schichtdicke ist in Abbildung 5.3 gezeigt. Die Ergebnisse von Ag auf glatten Si-Wafern (rot) wurden bereits in Kapitel 4.2 ausführlich diskutiert und erläutert. Die Rauigkeit der auf Si-Wellenstrukturen deponierten Filme steigt im gemessenen Schichtdickenbereich von etwa 1,2 nm auf etwa 1,6 nm minimal an, liegt aber stets in der Größenordnung der anfänglichen Substratrauigkeit von 1,4 nm. Im Bereich dünner Filme ist sie somit deutlich kleiner als bei den Schichten, die auf glattem Si deponiert wurden. Bei der Deposition auf glatten Substraten sind die Schichten ab etwa 8-10 nm geschlossen und die Rauigkeit nimmt einen minimalen Wert von etwa 0,8 nm an. Dieser Verlauf wird bei den auf Wellenstrukturen deponierten Filmen nicht beobachtet. Ursache hierfür ist, dass die Rauigkeit der Si-Wellen die der Ag-Inseln und später der geschlossenen Ag-Schichten überlagert. Dies bewirkt eine geringere Rauigkeit in den Anfangsstadien des Wachstums und eine größere Rauigkeit bei bereits geschlossenen Schichten. Die Rauigkeit des Substrats wurde demnach durch die Schichtdeposition nicht reduziert oder ausgeglättet.

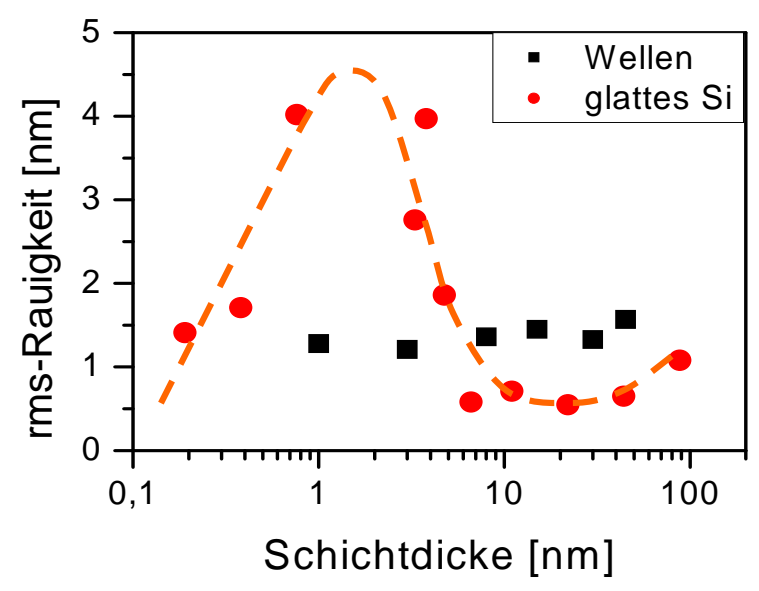

\begin{abstract}
Abbildung 5.3: Der Graph zeigt die Veränderungen der Rauigkeit mit der deponierten Ag-Schichtdicke, sowohl für glatte Si-Substrate (rot) also auch für die gewellten Si-Substrate (schwarz). Die gestrichelte Linie dient der Veranschaulichung.
\end{abstract}

Die Analyse der spektralen Leistungsdichten gibt Aufschluss über die vorherrschenden Strukturgrößen auf den jeweiligen Oberflächen (Abbildung 5.4). Aufgrund der Wellenstruktur weist das ursprüngliche Spektrum ein Hauptmaximum bei 1/(55 nm) und drei Nebenmaxima bei der doppelten, dreifachen und vierfachen Frequenz 
auf. Man beobachtet bei kleinen Schichtdicken zunächst einen kontinuierlichen Anstieg der Spektren im hochfrequenten Spektralbereich (vergleiche die Kurven für $1 \mathrm{~nm}$ und 3 nm Ag-Schichtdicke), der dazu führt, dass die hochfrequenten Maxima der ursprünglichen Wellenstrukturen einfach überlagert werden. Das Hauptmaximum, das der Periodizität der Wellen entspricht, nimmt etwas ab, ist aber weiterhin beobachtbar. Bei dickeren Schichten (vergleiche die Kurven ab 8 nm Schichtdicke) nehmen die kleinen Inselgrößen auf der Oberfläche (große Frequenzen im Spektrum) aufgrund von Koaleszenzprozessen und Säulenwachstum wieder ab. Hier wird auch das Hauptmaximum der Wellen von den größer werdenden Ag-Körnern der geschlossenen Schicht überlagert. Die spektralen Leistungsdichten der dicken Schichten zeigen mit einer Steigung von -5,6 einen steileren Abfall als die ursprünglichen Wellenstrukturen. Dies ist genau wie in Kapitel 3.3 beschrieben auf das kristalline Wachstum von Ag zurückzuführen.

Zusammenfassend haben die Experimente gezeigt, dass die dominanten Frequenzen des Si-Substrats, die die Rauigkeit bestimmen, durch die Deposition von Ag zwar verschwinden, stattdessen aber ähnlich große statistische Rauigkeiten aufgrund der AgInselstrukturen entstehen. Spektral gesehen haben sich nur die zur Rauigkeit beitragenden Strukturgrößen verändert. Die Eigenrauigkeit von Ag ist mit mehr als 0,8 nm somit zu groß.

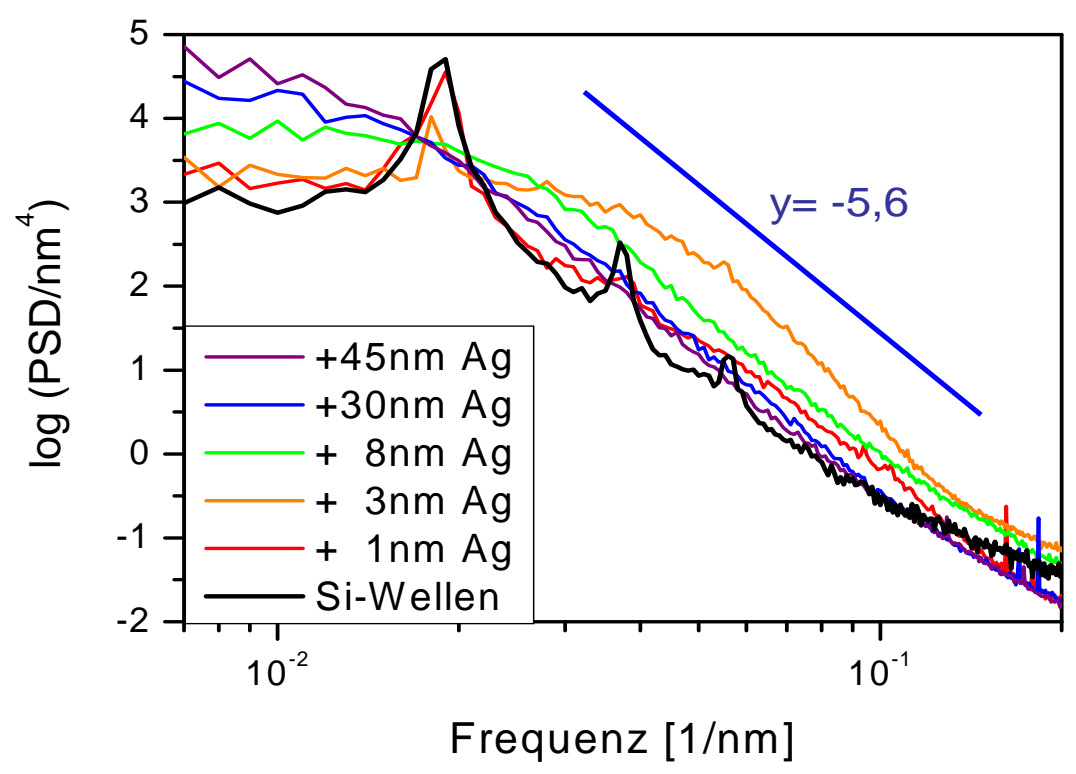

Abbildung 5.4: Veränderung der spektralen Leistungsdichte der Wellenstrukturen (schwarz) mit zunehmender Ag-Schichtdicke. 
Im Folgenden sollen daher Materialien zur Glättung verwendet werden, die eine geringere Eigenrauigkeit auf glatten Substraten haben.

\subsection{Glättung durch C}

Bevor die Glättung mit C diskutiert wird, soll die intrinsische Rauigkeit dieses Materials auf glatten Substraten untersucht werden. Dazu wurden Schichten unterschiedlicher Dicke auf Si-Wafern deponiert und ihre Morphologie mit einem AFM untersucht (Abbildung 5.5(a)-(d)). Die Filme haben unabhängig von ihrer Dicke eine Rauigkeit von etwa $0,15 \mathrm{~nm}$, allerdings steigt die charakteristische Strukturgröße der Oberfläche mit zunehmender Schichtdicke von etwa $25 \mathrm{~nm}$ auf etwa $38 \mathrm{~nm}$ an (Abbildung 5.5(e)).

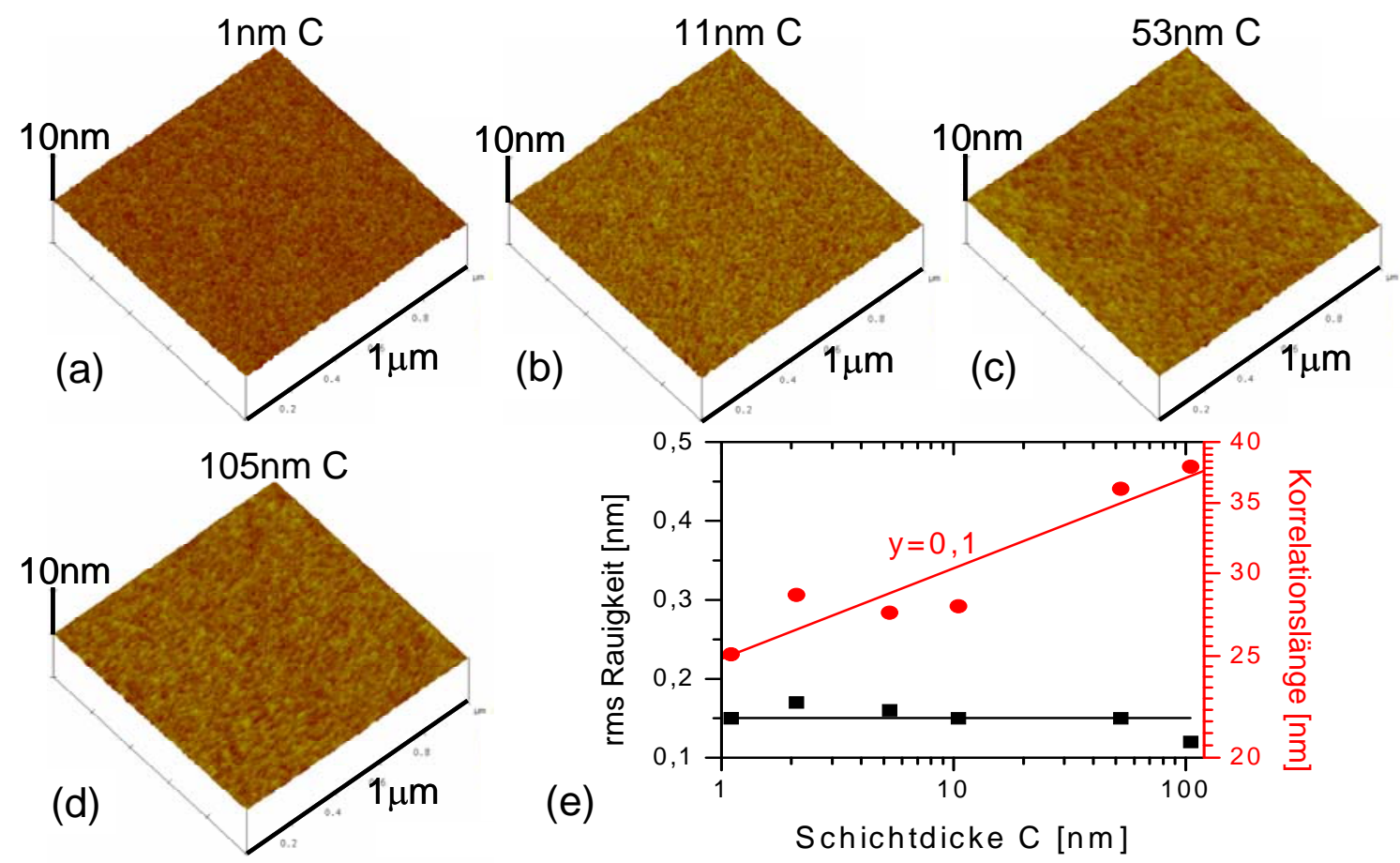

Abbildung 5.5: (a)-(d) AFM-Aufnahmen der Oberfläche von Kohlenstoffschichten unterschiedlicher Dicke deponiert auf glatten Si-Wafern. (e) zeigt die Veränderung der rms-Rauigkeit und der Korrelationslänge mit der Schichtdicke. 
Diese Korrelationslänge wurde aus den Abknickpunkten der jeweiligen spektralen Leistungsdichten bestimmt (Abbildung 5.6). Im hochfrequenten Bereich haben die Spektren dünner Schichten eine Steigung von etwa -5, ab einigen $10 \mathrm{~nm}$ Schichtdicke verringert sich der Wert jedoch auf $\mathbf{- 4}$. Dies spricht für die Bildung sehr kleiner, vermutlich amorpher Hügelstrukturen in den Anfangsstadien des Wachstums, die aufgrund von Diffusionsmechanismen nach und nach geglättet werden. Das führt zu einem Anwachsen der dominanten Strukturgröße (Korrelationslänge), wie es in Abbildung 5.5(e) zu sehen ist. Oberhalb dieser Korrelationslänge haben die Spektren nur noch eine Steigung von etwa -2. Hier sind Sputtererosion und ballistischer Transport die vorherrschenden Mechanismen, die zur Strukturbildung beitragen. Diese Interpretation deckt sich gut mit den Ergebnissen, die der Fit der Spektren liefert (Gleichung 3.13 unter Vernachlässigung der Mechanismen der Ordnung 1 und 3 (vergleiche Kapitel 3.3)). Bei der dicksten deponierten Schichtdicke erhält man eine kritische Wellenlänge von $(43 \pm 4) \mathrm{nm}$ : Strukturen bis zu dieser Größe werden hauptsächlich durch Diffusion [Herr50] erzeugt, ab dieser Größe vermehrt durch Sputtererosion [Brad88] und ballistischen Transport [Mose05]. Die Ergebnisse des Fits sind im Inset der Abbildung 5.6 dargestellt, die Werte für die $b_{\mathrm{i}}$ angegeben.

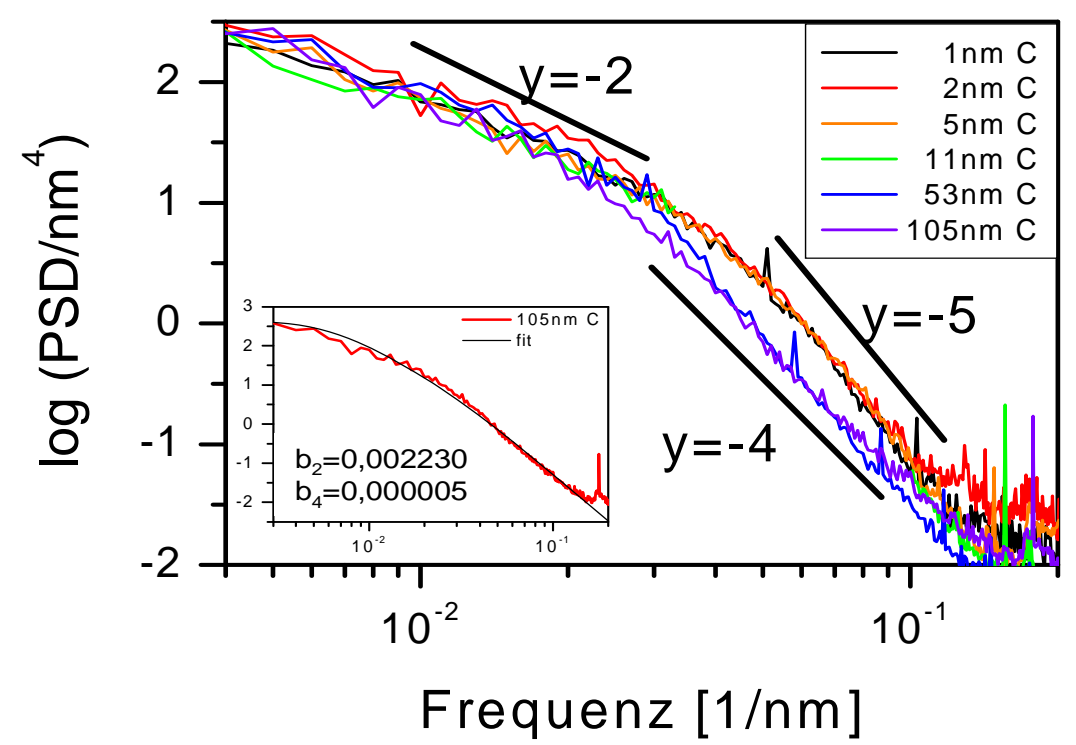

Abbildung 5.6: Spektrale Leistungsdichten der auf Si-Wafern deponierten CSchichten. Es gibt zwei Frequenzbereiche, in denen unterschiedliche Mechanismen dominieren. 
Damit erfüllt C im Gegensatz zu Ag das notwendige Kriterium einer geringen Eigenrauigkeit auf glatten Substraten. Um das Glättungsverhalten auf einer rauen Unterlage zu untersuchen, werden Schichten unterschiedlicher Dicke auf den beschriebenen Si-Wellenstrukturen deponiert. Die rasterkraftmikroskopischen Aufnahmen (Abbildung 5.7) zeigen, dass dies wie erhofft zu einer Verringerung der Höhe der SiWellen führt. Interessanterweise lässt sich hier die in der Theorie vorhergesagte frequenzabhängige Glättung beobachten: Zunächst werden kleine Unebenheiten wie beispielsweise die Strukturen auf den Wellenkämmen ausgeglättet. Die Hauptstruktur von $55 \mathrm{~nm}$ Wellenlänge nimmt sukzessive ab, ist aber auch bei $230 \mathrm{~nm}$ dicken C-Schichten noch nicht komplett verschwunden. Dickere Schichten konnten nicht deponiert werden, da diese aufgrund von hohen Spannungen und schlechter Haftung abplatzten.

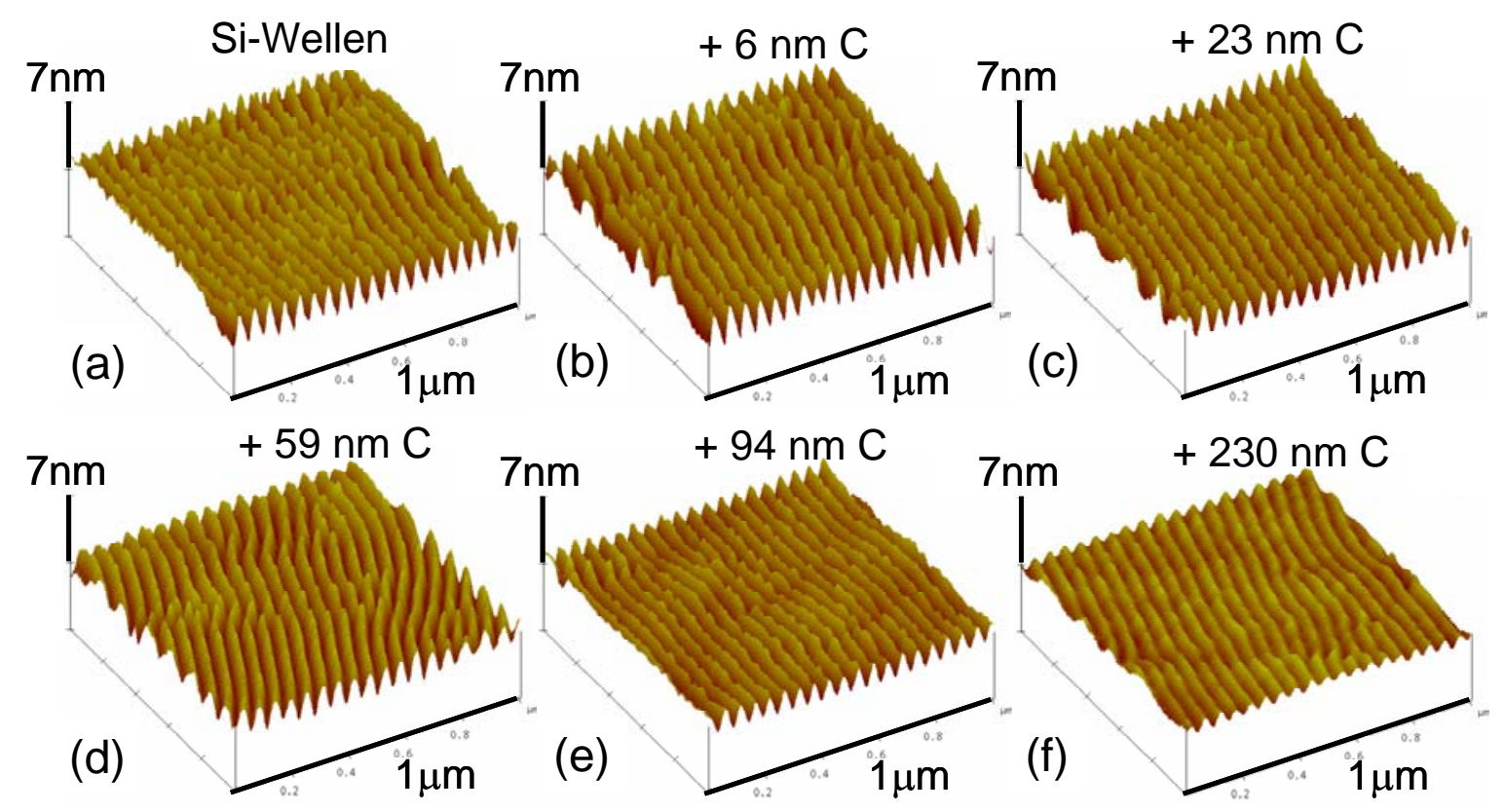

Abbildung 5.7: AFM-Aufnahmen der mit steigender Menge C beschichteten SiWellenstrukturen. Es lässt sich sowohl eine Glättung kleiner Strukturen auf den Wellenkämmen erkennen, als auch eine Abnahme der Strukturhöhe an sich.

Um die Abnahme der Höhe der Wellenstrukturen besser beobachten zu können, sind in Abbildung 5.8(a) die Höhenprofile der Proben dargestellt. Trotz der geringen Rauigkeit von C auf glatten Si-Wafern läuft die Glättung mit C sehr langsam ab, so dass eine vollständige Glättung der Strukturen auch bei $230 \mathrm{~nm}$ dicken Schichten nicht erreicht werden konnte. 

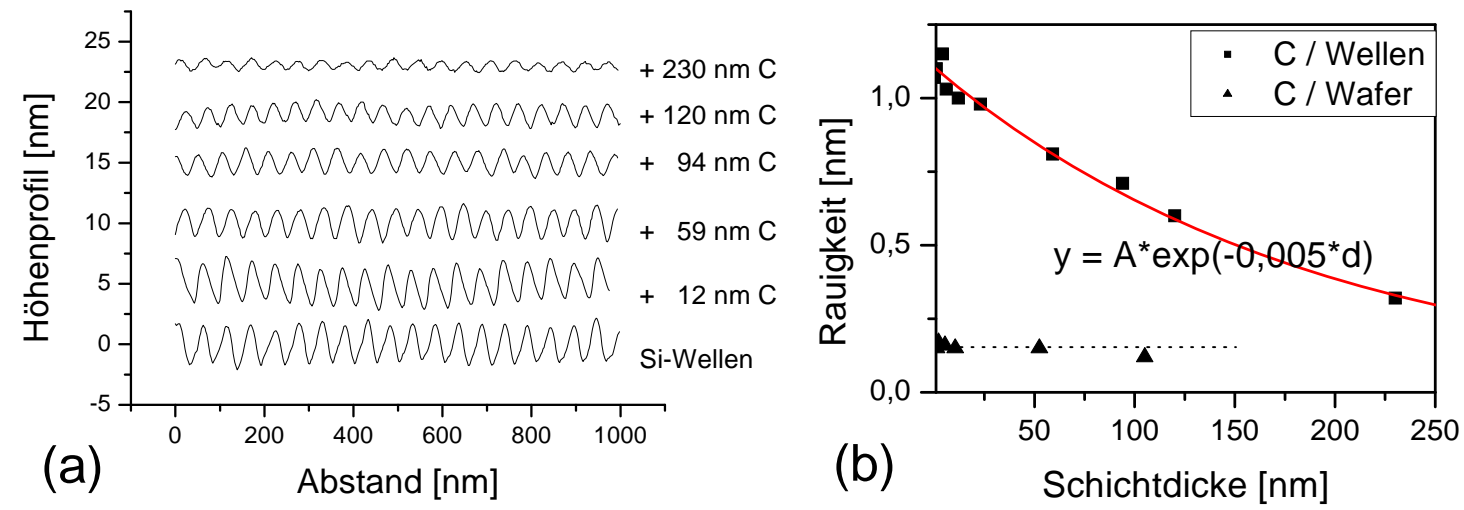

\begin{abstract}
Abbildung 5.8: (a) Übereinander angeordnete Höhenprofile der mit C beschichteten Wellenstrukturen. (b) Abnahme der rms-Rauigkeit mit steigender Schichtdicke C.
\end{abstract}

Die Analyse der rms-Rauigkeit von $1 \mathrm{x} 1 \mu \mathrm{m}^{2}$ großen Bildausschnitten zeigt einen exponentiellen Abfall der Rauigkeit. Dieses Verhalten entspricht den Erwartungen für Oberflächen, bei denen nur eine Frequenz in der spektralen Leistungsdichte auftritt (siehe auch Abschnitt 3.5), bzw. stark dominiert. Der Vorfaktor des Exponenten entspricht der doppelten Summe der gewichteten Potenzen von $k$ : const $=2 \sum b_{i} k^{i}$. Dieser Vorfaktor von 0,005 ist hier relativ klein, was die sehr langsame Glättung belegt. Abbildung 5.9 zeigt, wie sich die spektrale Leistungsdichte der Si-Wellenstrukturen mit zunehmender Menge C verändert. Wie theoretisch erwartet und bereits bei den AFM-Bildern vermutet, werden zunächst die hohen Frequenzen, d.h. die Maxima höherer Ordnung geglättet, bevor das Hauptmaximum in seiner Höhe reduziert wird. Des Weiteren beobachtet man ein scheinbar frequenzunabhängiges Absinken der Spektren mit zunehmender Schichtdicke, ein Verhalten, das noch häufiger auftreten wird, dessen Ursache aber bisher ungeklärt ist. Eine genauere Diskussion folgt in Abschnitt 5.5.

Für geringe Schichtdicken sind die Steigungen der Spektren ähnlich denen von C auf glatten Si-Wafern und eine genauere Analyse durch Anfitten dieser Spektren mit Gleichung 3.13 liefert eine Grenz-Strukturgröße von etwa $35 \mathrm{~nm}$, bei der sich der dominierende Glättungsmechanismus ändert. Interessant ist, dass sich die Steigung im hochfrequenten Teil auch bei dicken Schichten noch verändert, obwohl dort nur noch die vom Rauschterm erzeugten Rauigkeiten des aufwachsenden Films zur Endrauigkeit 
beitragen sollten. Ab einer Schichtdicke von etwa $120 \mathrm{~nm} \mathrm{C}$ aber erhöht sich die Steigung auf -2,6. Der Fit (siehe Inset) und die Analyse ergeben im Vergleich zu den Schichten auf glatten Substraten deutlich veränderte $b_{\mathrm{i}}$-Werte, deren Ursache möglicherweise der Aufbau starker Spannungen ist, die bei dickeren Filmen zum Abplatzen der Schichten führen.

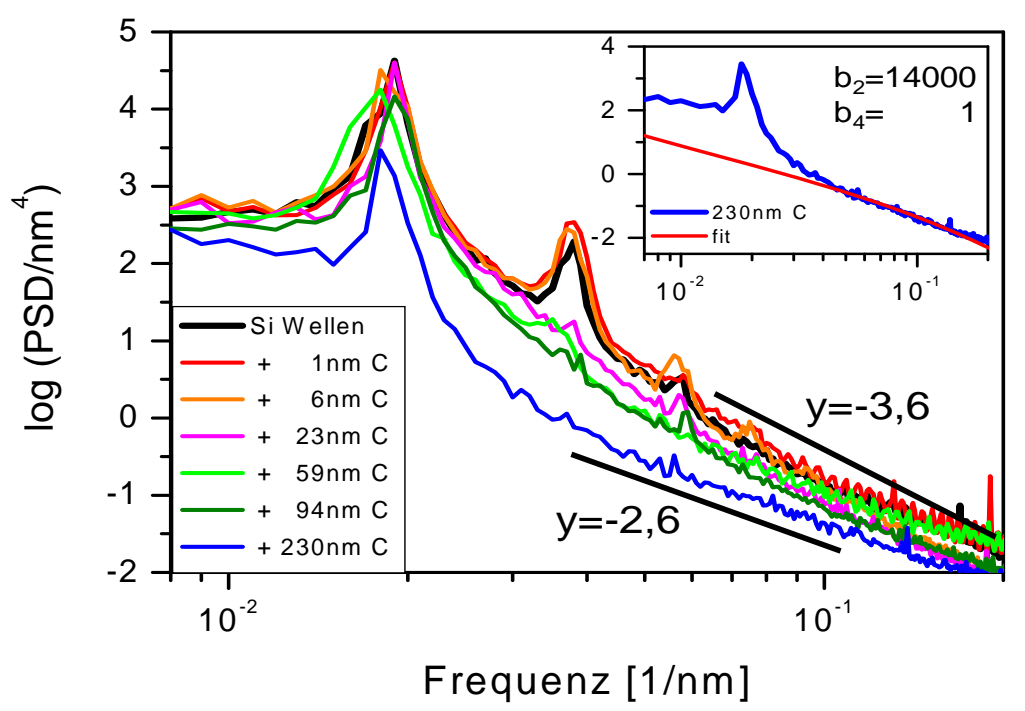

Abbildung 5.9: Veränderung der spektralen Leistungsdichte der Si-Wellenstrukturen nach der Beschichtung mit unterschiedlichen Mengen C.

Zusammenfassend lässt sich sagen, dass C zwar eine sehr geringe Eigenrauigkeit hat und damit im Gegensatz zu Ag die Grundvoraussetzungen erfüllt, sich allerdings aufgrund der schlechten Haftungseigenschaften und der sehr langsamen Glättung nicht als Glättungsmaterial für Si-Oberflächen eignet.

\subsection{Glättung durch PMMA}

Als erster Vertreter komplexer Materialien soll nun PMMA zur Glättung verwendet werden. Frühere Experimente haben gezeigt, dass sich dieses Polymer bei geringen Energiedichten mit gepulster Laserdeposition herstellen lässt. Die Schichten sind chemisch mit dem Ausgangsmaterial vergleichbar und sehr glatt [Löse06]. Die Herstellungs- 
parameter sind in Kapitel 2.1 erläutert. Um die Entwicklung der Morphologie auf glatten Si-Wafern zu beobachten, wurden erneut rasterkraftmikroskopische Bilder unterschiedlich dicker PMMA-Filme aufgenommen (Abbildung 5.10). Im Falle von noch nicht geschlossenen Schichten (4 nm Dicke, Abbildung 5.10(a)) beobachtet man die Bildung von etwa $130 \mathrm{~nm}$ breiten, bis zu $50 \mathrm{~nm}$ hohen Inseln. Dazwischen sind kleinere Inseln, die bereits perkolierende Strukturen zeigen. Bei dickeren Schichten sind die hohen Inseln weiterhin zu sehen, der Film dazwischen ist allerdings geschlossen und sehr glatt. Mit steigender Schichtdicke nimmt die Höhe ab und die Breite zu, bis schließlich ab etwa 200 nm Dicke nur noch ein sehr glatter Film zurückbleibt, der auch nicht wieder aufraut. Dieses Verhalten lässt darauf schließen, dass die Inseln in der Anfangsphase des Wachstums gebildet werden und danach keine neuen mehr entstehen, sondern die vorhandenen nach und nach geglättet werden.

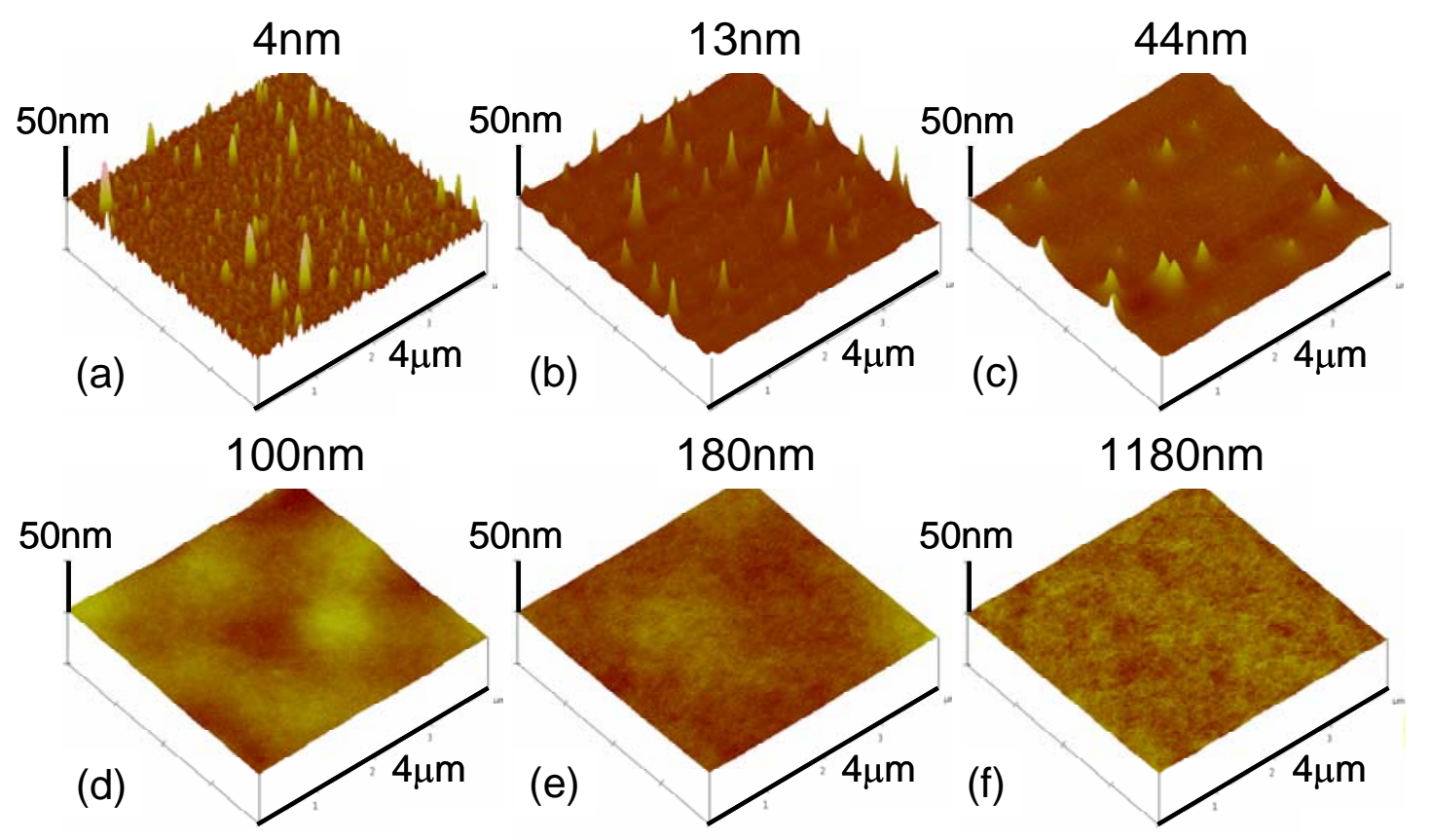

Abbildung 5.10: AFM-Bilder von PMMA-Filmen auf glatten Si-Wafern. Die deponierten Schichtdicken sind angegeben. Für den besseren Vergleich wurde für alle Bilder eine gemeinsame Höhenskala von $50 \mathrm{~nm}$ gewählt. In Abbildung 5.12 ist beispielhaft eine der glatten Schichten mit kleinerer Skala dargestellt.

Die Höhenprofile unterschiedlich dicker Filme zeigen, dass sich die Krümmung der Inselflanken ändert (Abbildung 5.11). Während bei dem noch nicht geschlossenen, $4 \mathrm{~nm}$ 
dicken Film die Inseln Kugelkappen ähneln und die Krümmung der Flanken konvex ist, ändert sich dies, sobald die Schichten geschlossen sind. Hier zieht das PMMA sich langsam glatt, um die Oberflächenenergie zu minimieren und die Krümmung der Flanken wird konkav. Eine mögliche Ursache für die hohen Inseln in den Anfangsstadien des Wachstums ist Entnetzung. Ein Hinweis hierfür ist die konvexe Krümmung der Inseln auf der noch nicht vollständig bedeckten $\mathrm{SiO}_{2}$-Oberfläche. Hierbei können auch leichte Verunreinigungen des Substrats eine Rolle gespielt haben, da die Oberfläche aufgrund der geringen verwendeten Depositionsenergie nicht automatisch durch Absputtereffekte gesäubert wurde. Eine Analyse der Statik und Kinetik bei Entnetzung findet sich bei [Baue06].

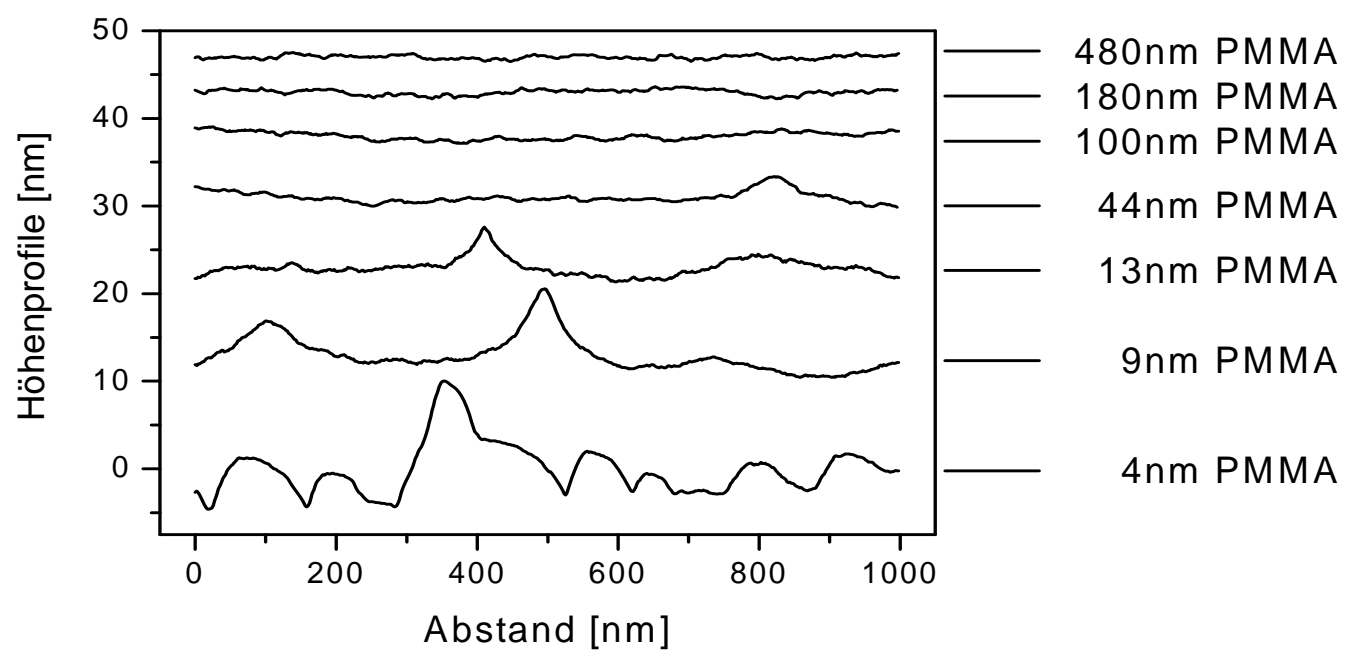

Abbildung 5.11: Übereinander angeordnete Höhenprofile unterschiedlich dicker PMMA-Filme auf glatten Si-Wafern.

Der Verlauf der Rauigkeit mit zunehmender Schichtdicke (Abbildung 5.12(a)) zeigt, dass die Schichten anfänglich aufgrund der starken Inselbildung bei der Entnetzung sehr rau sind. Mit steigender Schichtdicke nehmen die Werte zunächst nur langsam ab, dann wird die Oberfläche jedoch schnell geglättet, bis schließlich eine Endrauigkeit von etwa 0,3 nm erreicht ist. Abbildung 5.12(b) zeigt noch mal die Morphologie einer solchen glatten Schicht mit geeigneter Höhenskala, da die Bilder aus Abbildung 5.10 aufgrund der zum Vergleich notwendigen großen z-Skala schwerer interpretierbar sind. Es zeigt sich, 
dass auf dem gewählten Bildausschnitt keine spezielle Hügelgröße dominiert, sondern unterschiedlichste Strukturgrößen sichtbar sind.
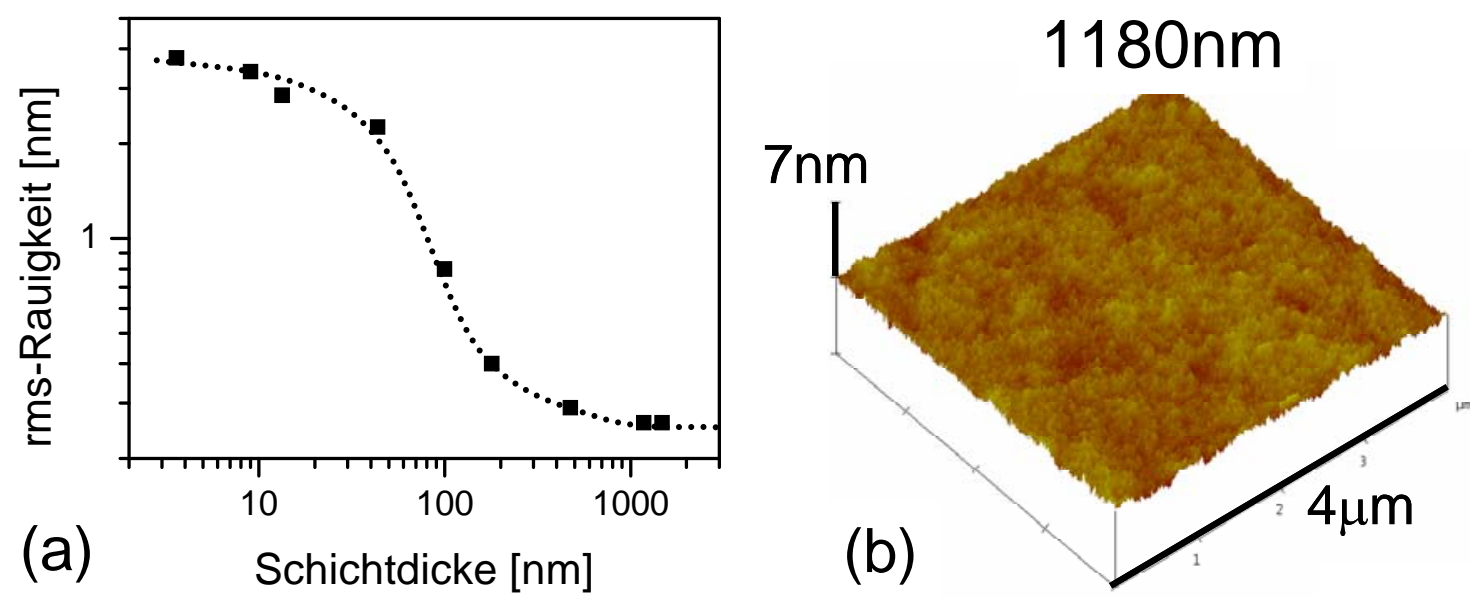

Abbildung 5.12: (a) Veränderung der Rauigkeit mit zunehmender Schichtdicke.

(b) AFM-Bild einer 1180 nm dicken PMMA-Schicht mit kleiner Höhenskala, die einen besseren Eindruck von der Oberflächenmorphologie bietet als Abbildung 5.10.

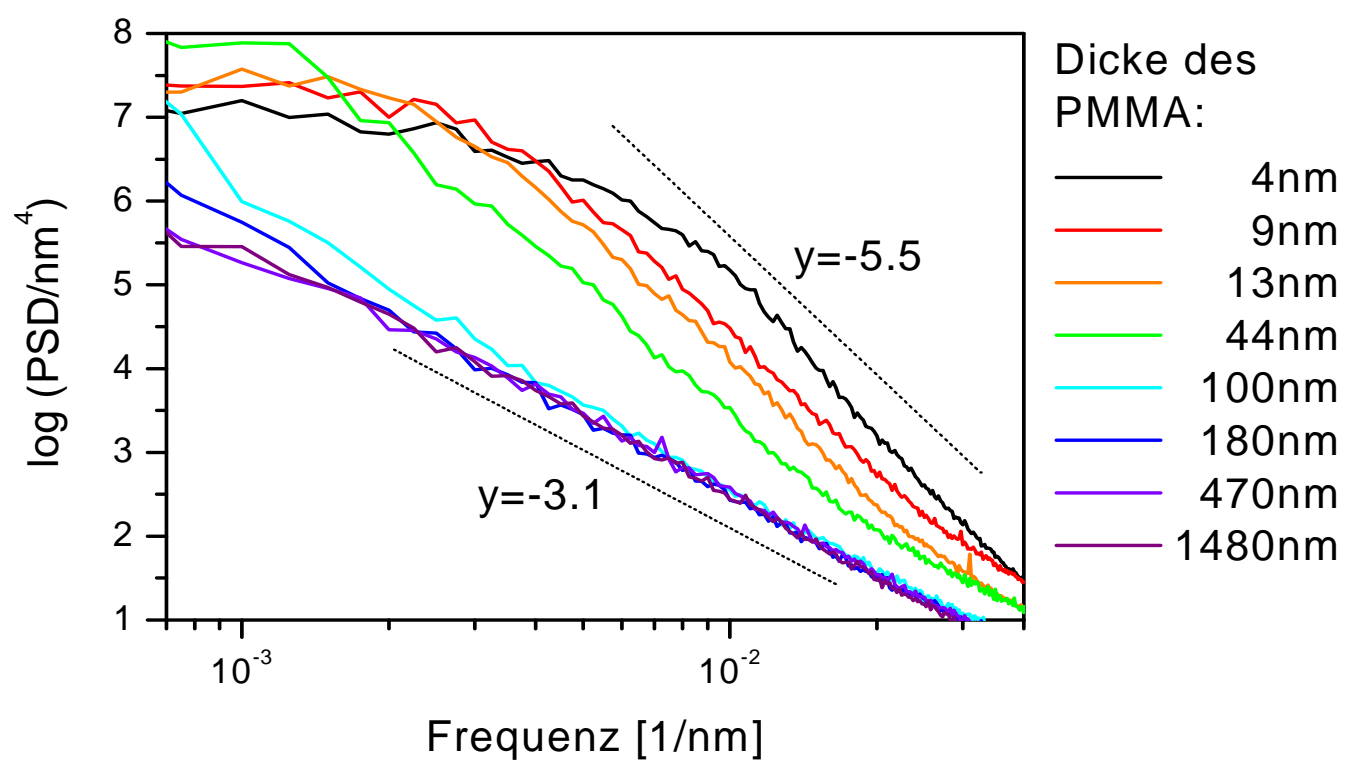

Abbildung 5.13: Spektrale Leistungsdichten der PMMA-Filme unterschiedlicher Schichtdicke. 
Mithilfe der spektralen Leistungsdichten aus Abbildung 5.13 lassen sich das Glättungsverhalten und der Verlauf der Rauigkeiten besser verstehen. Das schwarze Spektrum gehört zu dem noch nicht geschlossenen Film. Aus dem relativ breiten Abknickbereich lässt sich die Inselgröße bestimmen. Sie liegt zwischen $100 \mathrm{~nm}$ und $300 \mathrm{~nm}$. Mit zunehmender Schichtdicke werden die Strukturen auf der Oberfläche langsam ausgeglättet. Dies geschieht, wie in der Theorie beschrieben, frequenzabhängig. Zunächst werden hohe Frequenzen geglättet, später niedrige. Die Rauigkeit der Oberfläche selbst wird anfangs nur langsam reduziert, da die Glättung der hohen Frequenzen nur einen geringen Beitrag zur Gesamtrauigkeit liefert. Erst wenn die zur Inselbreite gehörenden Frequenzen im Spektrum deutlich reduziert werden (bei etwa $100 \mathrm{~nm}$ deponierter Schichtdicke), nimmt auch die Gesamtrauigkeit der Oberfläche deutlich ab.

Aufgrund seiner geringen Eigenrauigkeit nach dem Glattziehen der Filme eignet sich PMMA folglich prinzipiell zur Glättung rauer Oberflächen, allerdings muss im Fall von Si als Substrat die Entnetzung und damit verbundene Aufrauung bei Schichten unter $200 \mathrm{~nm}$ Schichtdicke in Kauf genommen werden. Im Folgenden soll PMMA auf SiWellenstrukturen deponiert werden, um das Glättungsverhalten zu untersuchen.

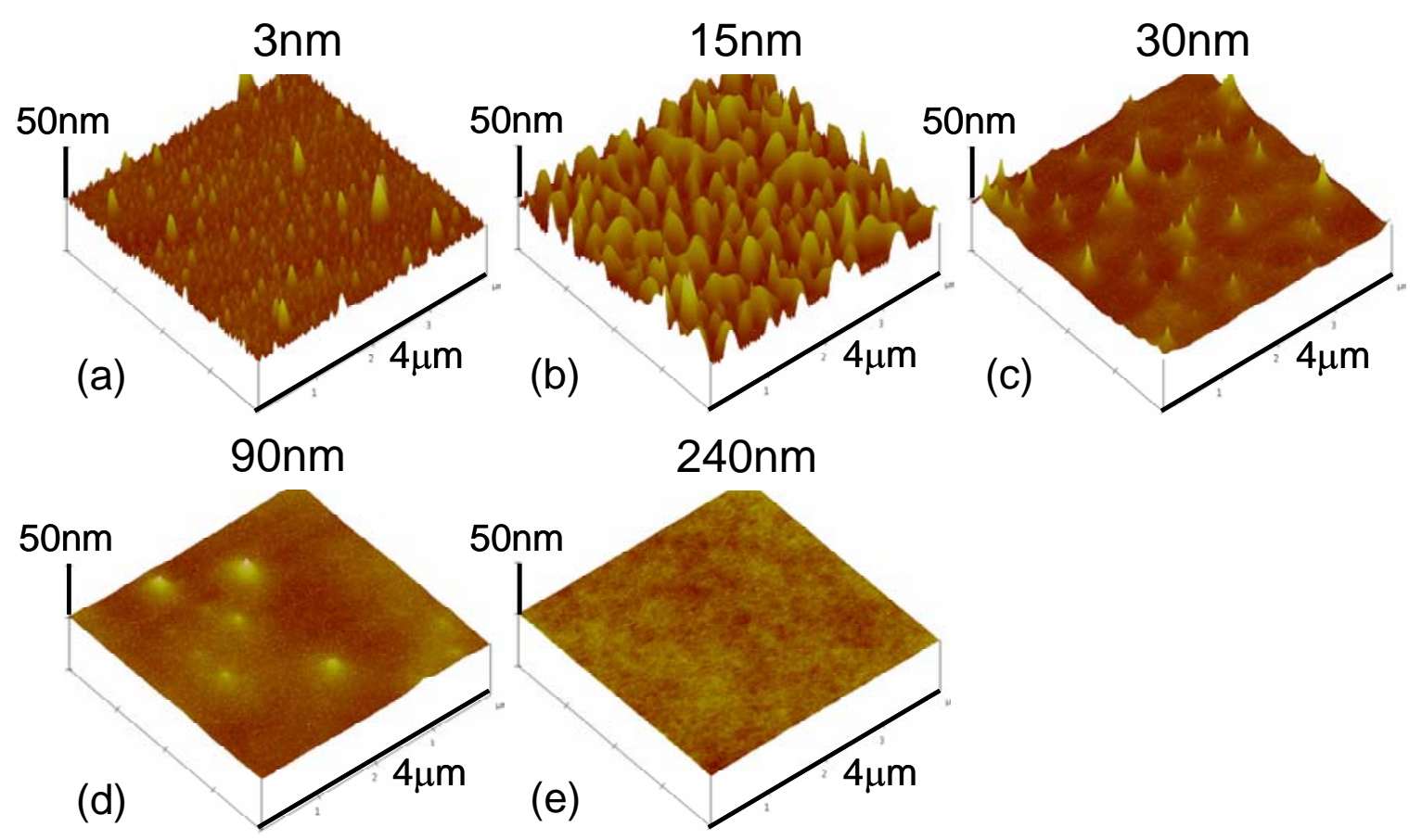

Abbildung 5.14: AFM-Aufnahmen unterschiedlich dicker PMMA-Schichten auf gewellten Si-Substraten. 
Die Oberflächenmorphologie der gewellten Substrate nach Deposition unterschiedlich dicker PMMA-Schichten wurde ebenfalls mit dem AFM untersucht (Abbildung 5.14). Ähnlich wie bereits auf den glatten Substraten beobachtet, entstehen bei Schichtdicken von wenigen Nanometern sehr hohe Inseln, die sich glatt ziehen, sobald die Filme geschlossen sind. Interessanterweise treten hier bei $15 \mathrm{~nm}$ Schichtdicke noch stark perkolierende Strukturen auf, während auf glatten Substraten die Filme bei einer Dicke von $13 \mathrm{~nm}$ bereits geschlossen waren. Dies lässt darauf schließen, dass die Entnetzung von der Substratrauigkeit beeinflusst wird und auf rauen Substraten deutlich stärker ist. Ähnliche Beobachtungen bestätigen auch [Rehs01]. Die Oberfläche der mit $90 \mathrm{~nm}$ PMMA bedeckten Wellenstruktur zeigt noch einige Inseln sehr geringer Höhe, es ist aber zu vermuten, dass auch hier bei Dicken von etwa $200 \mathrm{~nm}$ die Glättung vollständig ist. Bei diesen dicken Schichten beobachtet man dieselben Endrauigkeiten von etwa 0,3 nm wie schon bei der Deposition auf glatten Substraten.

Da sich bei den gezeigten 3D-Aufnahmen und dem großen Bildausschnitt die gewellten Strukturen schlecht erkennen lassen, ist in Abbildung 5.15 die Oberflächenmorphologie farbkodiert in Draufsicht dargestellt. Die Höhenskalen sind angegeben. Die Aufnahme der Oberfläche der 3 nm dicken Schicht in Abbildung 5.15(a) zeigt, dass sich die PMMA-Inseln gleichmäßig über die Oberfläche verteilen, sich allerdings bevorzugt in den Wellentälern anordnen, bis sie so groß werden, dass sie mehrere Hügel und Täler überspannen.
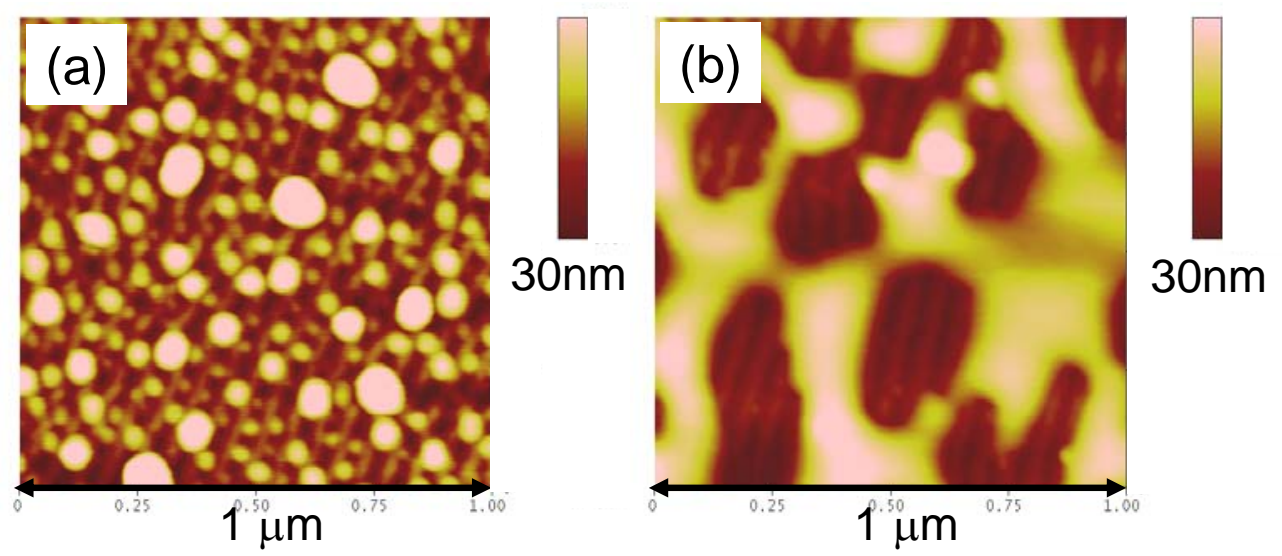

Abbildung 5.15: Die AFM-Bilder zeigen (a) die $3 \mathrm{~nm}$ und (b) die $15 \mathrm{~nm}$ dicken PMMA-Schichten aus Abbildung 5.14 in Draufsicht. Die Höhenskala ist farbkodiert und im Bild angegeben. Hier lässt sich die gewellte Si-Oberfläche gut von der Schicht unterscheiden. 


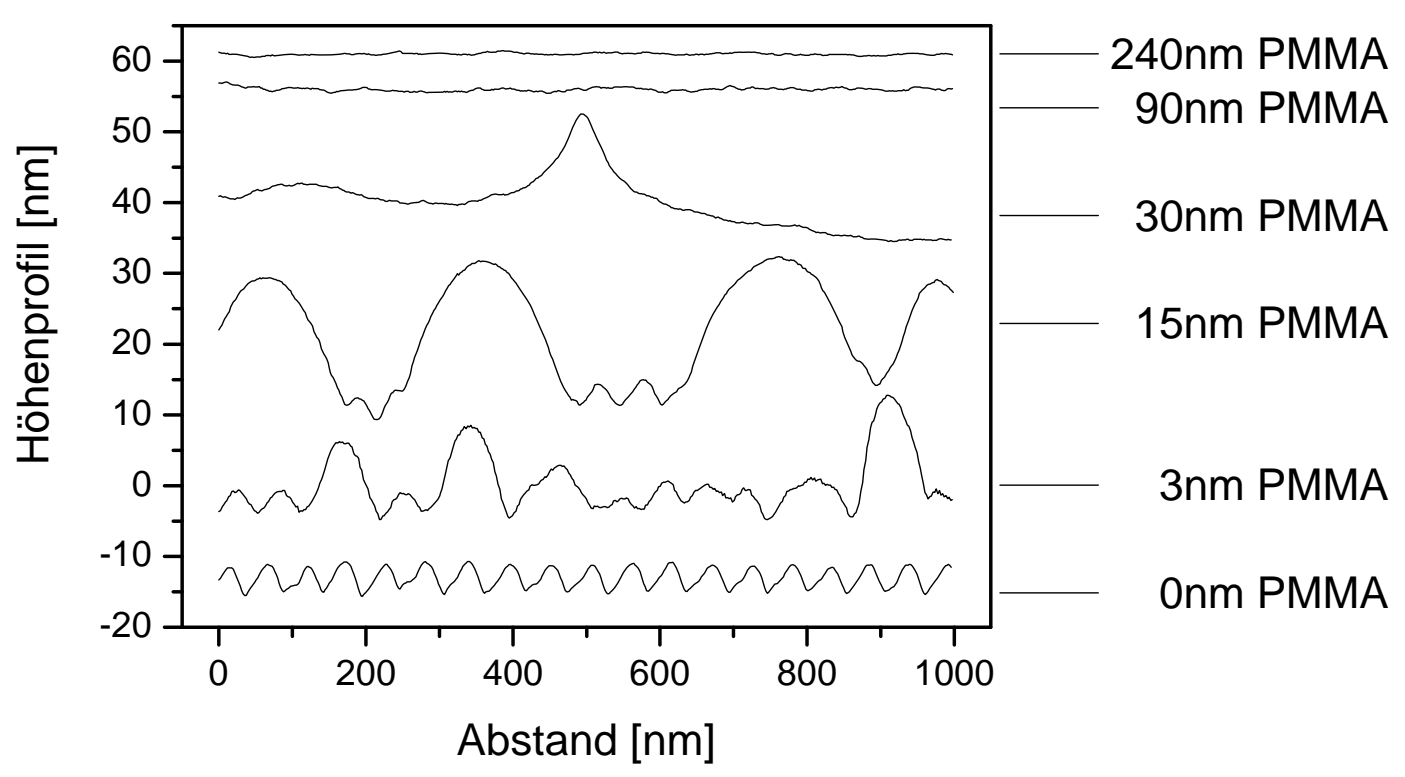

Abbildung 5.16: Übereinander dargestellte Höhenprofile unterschiedlich dicker PMMA-Schichten deponiert auf gewellten Si-Substraten.

Die Oberfläche der mit 15 nm PMMA bedeckten Probe (Abbildung 5.15(b)) zeigt perkolierende Strukturen, zwischen denen relativ große, unbedeckte Bereiche des gewellten Substrats zu sehen sind. Diese Morphologie ist ebenfalls ein Hinweis auf die entnetzenden Eigenschaften des Polymers. Zur besseren Übersicht sind in Abbildung 5.16 die Höhenprofile unterschiedlich dicker PMMA-Schichten übereinander angeordnet dargestellt. Die Graphen bestätigen die bisherigen Beobachtungen. Sie zeigen ebenfalls die bereits für das Wachstum auf glatten Substraten beschriebene Krümmungsänderung der Hügelflanken, die auf unterschiedliche Mechanismen bei der Strukturbildung hindeuten.

Der Verlauf der rms-Rauigkeiten mit der deponierten Schichtdicke ist in Abbildung 5.17 dargestellt. Die Balken auf der x-Achse markieren die anfängliche Rauigkeit des Substrats. Der Verlauf der Kurven ist für beide Substratarten ähnlich. Zunächst steigt die Rauigkeit aufgrund der Inselbildung und Entnetzung an und nimmt dann erst deutlich ab, wenn die Inselhöhe durch Glattziehen der Schichten drastisch reduziert wurde. Die anfängliche Aufrauung ist für die gewellten Substrate höher, und somit die Entnetzung stärker. Die Endrauigkeit liegt stets bei etwa 0,3 nm. 


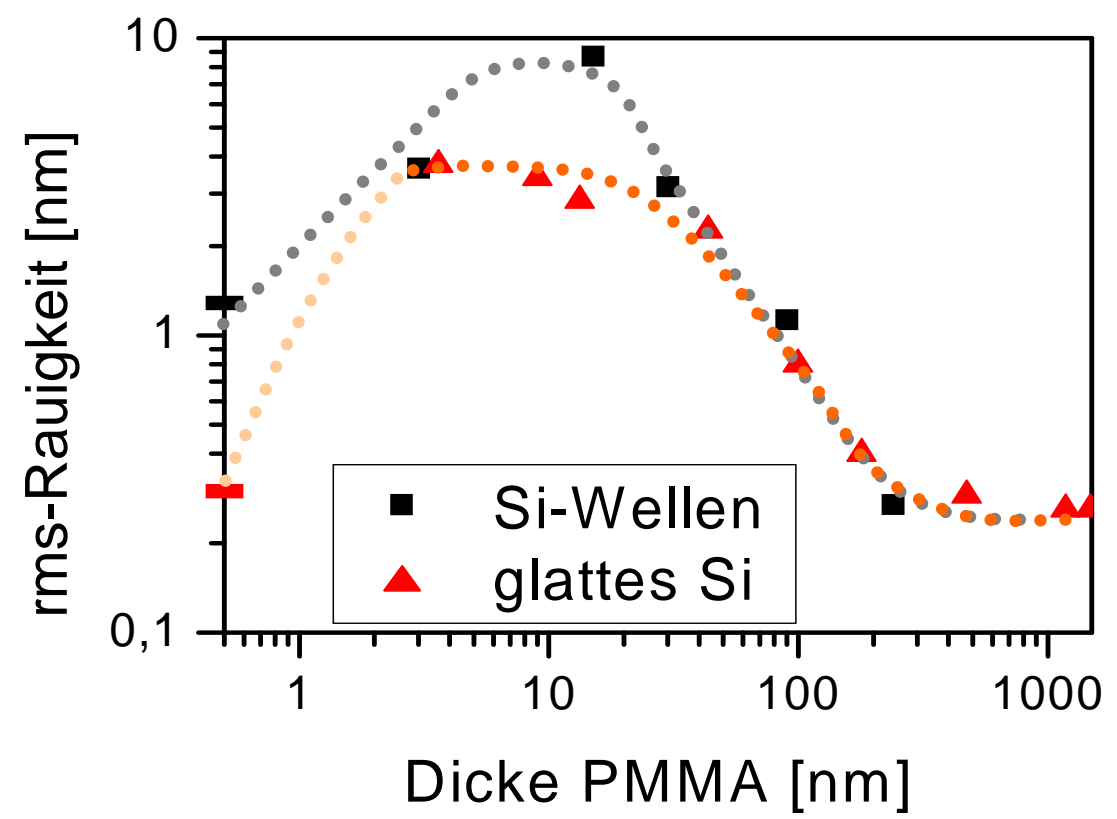

Abbildung 5.17: Verlauf der rms-Rauigkeiten in Abhängigkeit der deponierten PMMA-Schichtdicke. Die Balken auf der x-Achse markieren die jeweiligen Anfangsrauigkeiten der Substrate. Die gepunkteten Linien sind Hilfslinien, die der Veranschaulichung dienen sollen.

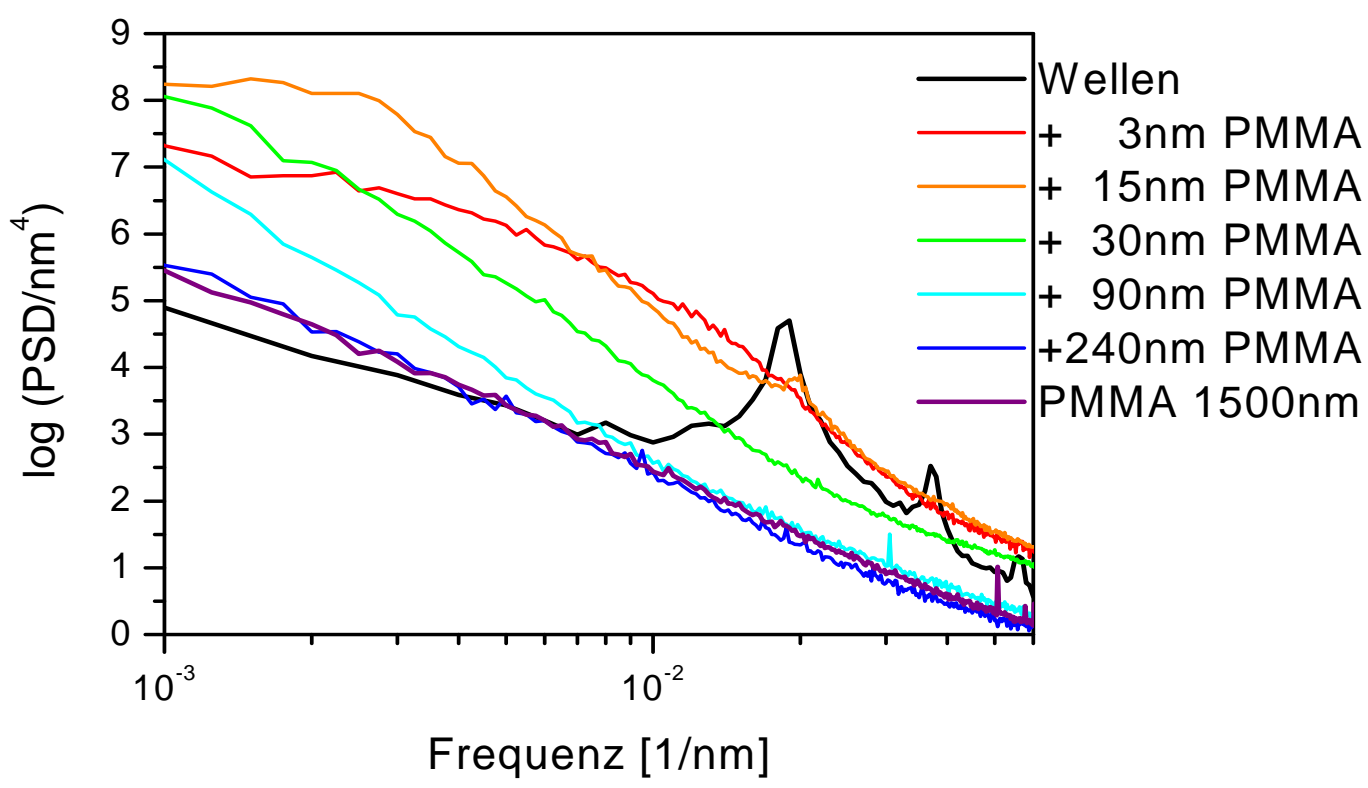

Abbildung 5.18: Veränderung der spektralen Leistungsdichte der Si-Wellenstrukturen (schwarz) mit zunehmender Menge PMMA. Die $1500 \mathrm{~nm}$ dicke Schicht wurde auf glattem Si deponiert. 
Wie durch die Oberflächenmorphologie erwartet, steigt die spektrale Leistungsdichte der Proben bei dünnen PMMA-Schichten zunächst stark an (Abbildung 5.18). Das Spektrum der $3 \mathrm{~nm}$ dicken Schicht (rot) zeigt keine Maxima mehr, die einen Hinweis auf die Wellenstrukturen geben würden, und knickt im Wellenlängenbereich von $60 \mathrm{~nm}$ bis $180 \mathrm{~nm}$ ab. In dieser Größenordnung liegt die mittlere Inselgröße auf den Proben. Diese überdecken die Periodizität des strukturierten Si weitgehend. Bei der $15 \mathrm{~nm}$ dicken Probe haben bereits die AFM-Aufnahmen gezeigt, dass wieder verstärkt die ursprüngliche Si-Oberfläche freigelegt wird, was man auch im Spektrum sieht: Die zur Periodizität der Wellen gehörenden Maxima kommen wieder leicht zum Vorschein. Je mehr sich die anfänglich gebildeten Hügel mit zunehmender Schichtdicke glatt ziehen, desto stärker sinken die Spektren ab, bis sie auf dem erwarteten Endspektrum einer dicken PMMA-Schicht auf glattem Substrat liegen. Interessanterweise ist dieser Glättungsprozess ebenfalls frequenzabhängig, was sich besonders gut verfolgen lässt, wenn man die Kurven der 30 nm, 90 nm und 240 nm dicken Schichten vergleicht.

Abschließend lässt sich sagen, dass Si-Oberflächen gut mit PMMA geglättet werden können, sofern man ein anfängliches Aufrauen durch Entnetzungsphänomene in Kauf nimmt. Des Weiteren darf die ursprüngliche Oberfläche nicht zu rau sein, da die Rauigkeit die Entnetzung beeinflusst [Rehs01]. Die zur Glättung notwendige Schichtdicke beträgt etwa $200 \mathrm{~nm}$.

\subsection{Glättung durch PC}

Als zweites Polymer soll PC als glättendes Material verwendet werden. Auch hier haben bereits frühere Arbeiten gezeigt, dass ein mit PLD hergestellter PC-Film gute Haftungseigenschaften hat und relativ glatt ist [Faup05, Röde08]. Um die Eigenrauigkeit und die Oberflächenbeschaffenheit von PC schichtdickenabhängig zu bestimmen, wurden zunächst wieder PC-Schichten unterschiedlicher Dicke auf glatten Si-Wafern hergestellt und mit dem AFM untersucht (Abbildung 5.19). In den Anfangsstadien des Wachstums besteht der Film aus sehr kleinen Strukturen von nur etwa $30 \mathrm{~nm}$ Größe. Die Oberflächenrauigkeit beträgt etwa 0,2 nm. Es ist davon auszugehen, dass die Schichten 
hier bereits geschlossen sind. Mit steigender Schichtdicke wachsen diese amorphen Hügelstrukturen an und die Oberfläche wird etwas rauer.
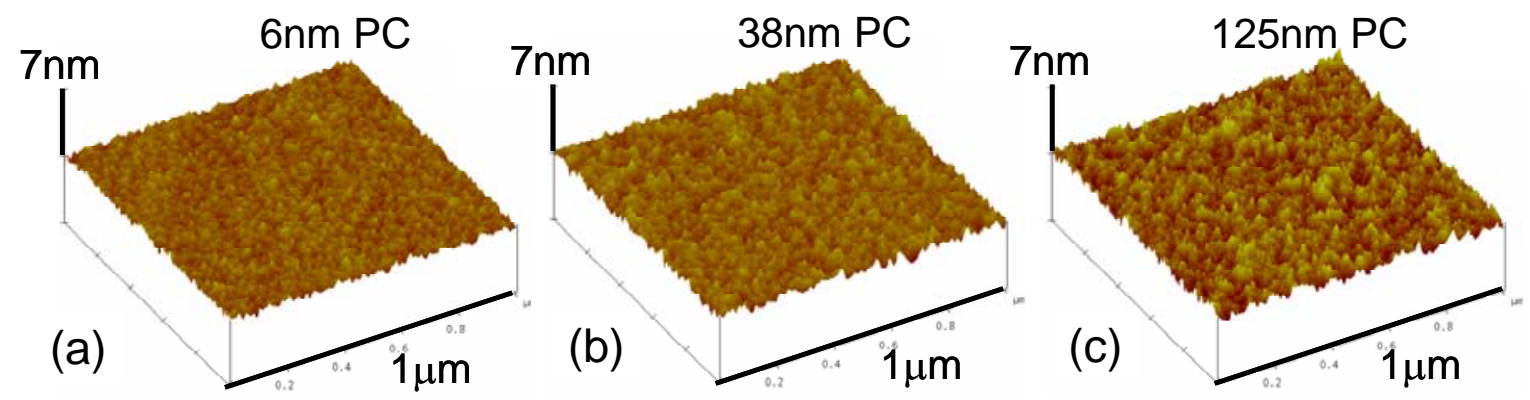

Abbildung 5.19: AFM-Aufnahmen von laserdeponierten PC-Filmen auf glatten Si-Substraten. Die jeweiligen Schichtdicken sind angegeben. Die Rauigkeit steigt leicht an von 0,2 nm (6 nm PC) über 0,3 nm (38 nm PC) auf 0,35 nm (125 nm PC), für eine graphische Darstellung siehe Abbildung 5.23.

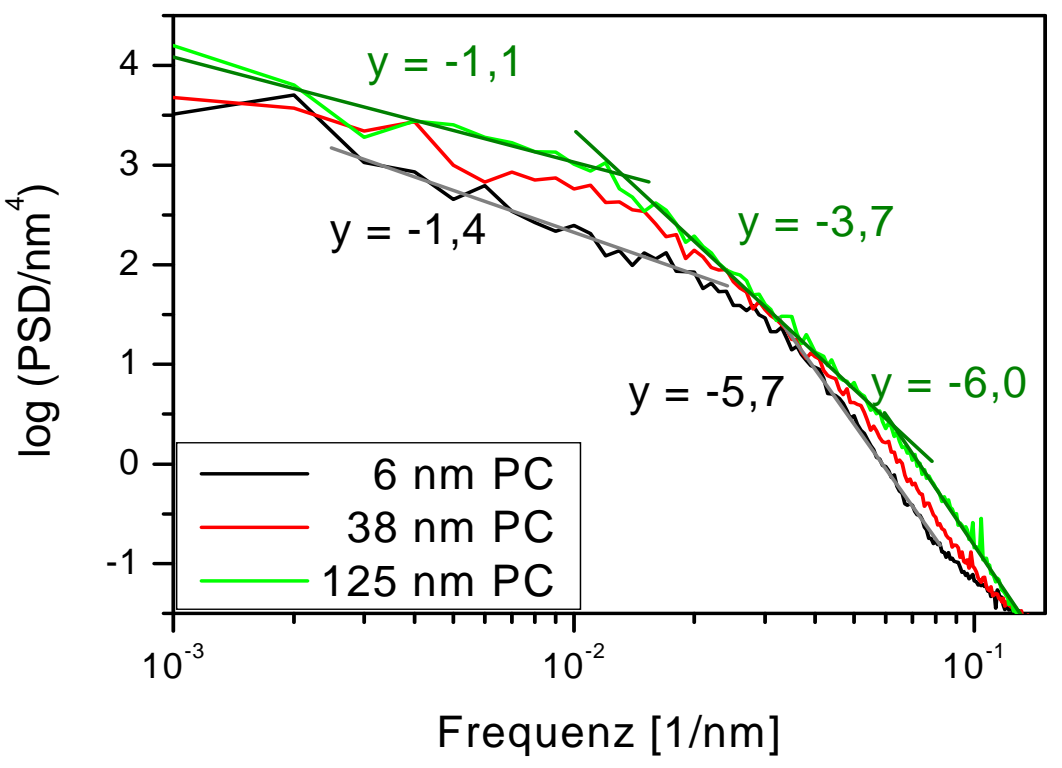

Abbildung 5.20: Der Graph zeigt die spektralen Leistungsdichten der PC-Filme auf glatten Si-Substraten. Mit zunehmender Schichtdicke beobachtet man einen leichten Anstieg der Kurven, was den Anstieg der Oberflächenrauigkeit zeigt.

Bei einer Schichtdicke von $125 \mathrm{~nm}$ PC beträgt die Rauigkeit etwa 0,35 nm und die Oberfläche besteht aus amorphen Strukturen sehr unterschiedlicher Größe. Im Gegensatz zu PMMA ist bei den PC-Schichten keine Entnetzung und eine damit verbundene Aufrauung zu beobachten. Die spektralen Leistungsdichten in Abbildung 5.20 steigen wie 
erwartet mit zunehmender Schichtdicke an. Dies ist Folge des geringfügigen Aufrauens der Oberfläche. Bei dem Graph der $6 \mathrm{~nm}$ dicken Schicht sieht man noch ein deutliches Abknicken bei einer Strukturgröße von etwa $30 \mathrm{~nm}$. Diese entspricht der charakteristischen Hügelgröße auf der Oberfläche. Mit steigender Schichtdicke bilden sich mehrere Abknickpunkte aus, die Inselgrößen von $20 \mathrm{~nm}$ und $80 \mathrm{~nm}$ entsprechen. Die Interpretation der Steigungen ist für Polymere schwierig. Im Falle von nichtpolymeren amorphen Materialien würden sich kleine Strukturen unter $20 \mathrm{~nm}$ durch inselartiges Wachstum bilden (Steigungen deutlich kleiner als -4 [Erle97, Eise01]), größere diffusionsgesteuert (Steigung von etwa -4 [Herr50]). Möglicherweise ist diese Interpretation hier auch zutreffend, da das Polymer durch den Ablationsprozess wahrscheinlich teilweise degradiert ist (vergleiche Kapitel 2.1) und die kleinen Bruchstücke sich ähnlich verhalten könnten wie Atome oder einfache Moleküle.

Da die PC-Schichten eine vergleichsweise geringe intrinsische Rauigkeit haben und nicht entnetzen, scheint sich PC als Glättungsmaterial zu eignen. Im Folgenden soll untersucht werden, wie sich laserdeponierte PC-Schichten auf gewellten Substraten verhalten und ob damit tatsächlich eine effiziente, d.h. schnelle Glättung der Oberfläche erreicht werden kann.
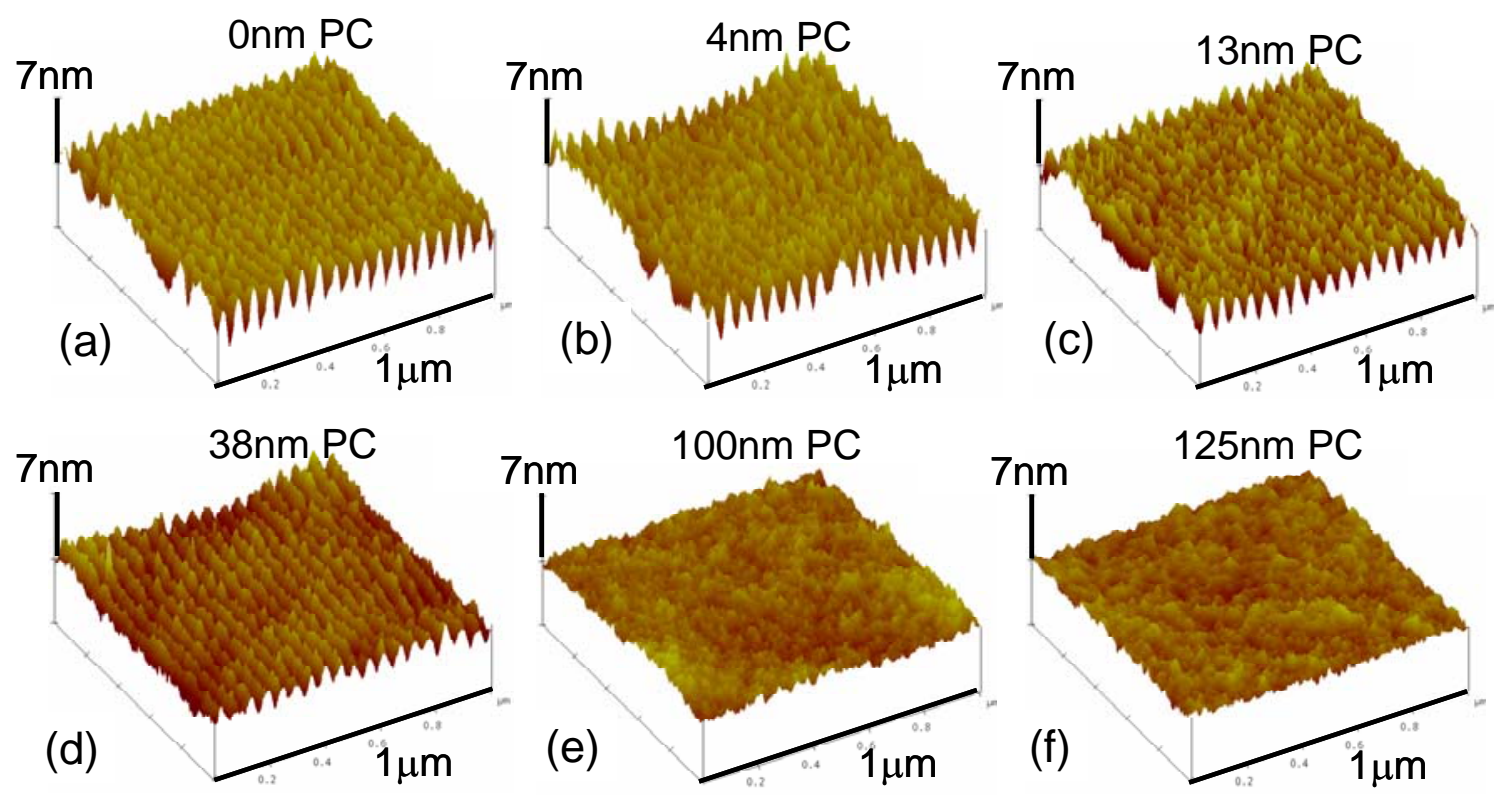

Abbildung 5.21: AFM-Bilder der Wellensubstrate (a) vor und (b)-(f) nach der Deposition von PC-Filmen mit unterschiedlicher Schichtdicke. 
In Abbildung 5.21 sind rasterkraftmikroskopische Bilder der Si-Wellenstrukturen vor und nach der Deposition der angegebenen Mengen PC dargestellt. Bis zu einer Schichtdicke von $38 \mathrm{~nm}$ sind die Wellenstrukturen noch erkennbar, aber ihre Höhe nimmt kontinuierlich ab. Der Vergleich der Oberflächenprofile (Abbildung 5.22) zeigt, dass bei einer Schichtdicke von $63 \mathrm{~nm}$ die Wellen komplett verschwunden sind. Es bleibt eine Endrauigkeit von 0,4 nm bestehen, die auch mit zunehmender Schichtdicke nicht mehr ausgeglättet werden kann.

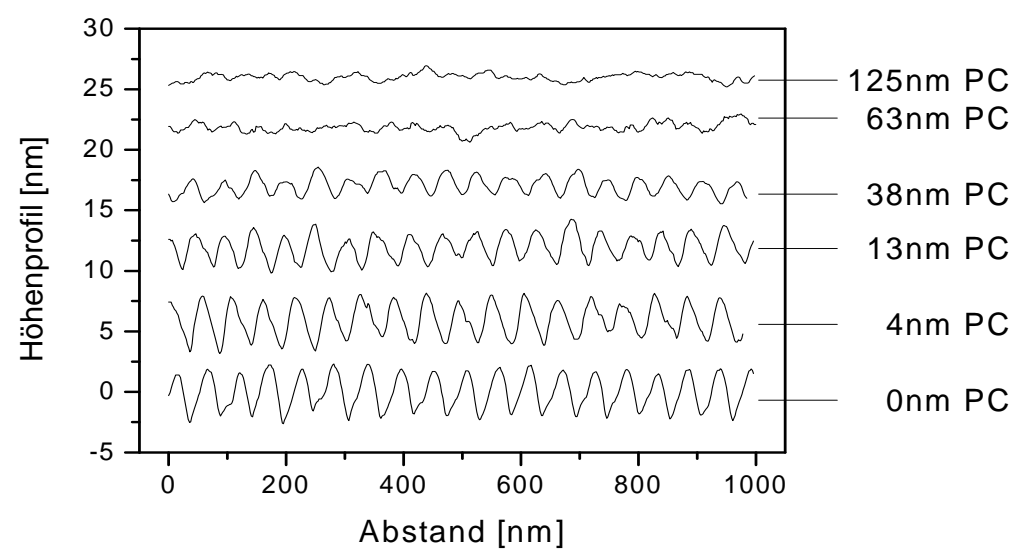

Abbildung 5.22: Übereinander angeordnete Höhenprofile der Si-Wellenstrukturen nach Deposition der angegebenen Mengen PC.

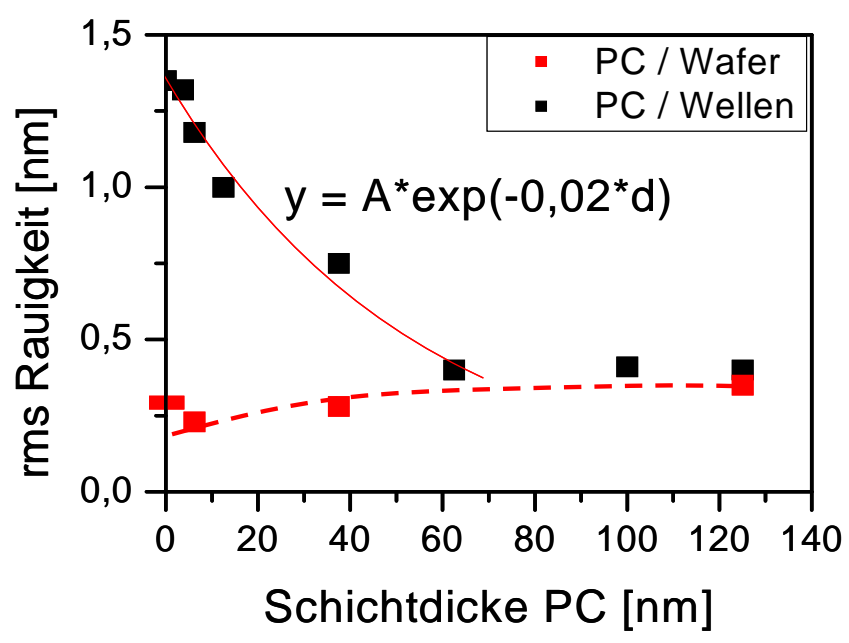

Abbildung 5.23: In rot ist die Entwicklung der intrinsischen Rauigkeit von PC auf glatten Si-Substraten dargestellt, in schwarz die Abnahme der Rauigkeit der Si-Wellen mit steigender Schichtdicke. Der exponentielle Abfall wurde gefittet, während die gestrichelte Linie nur der Veranschaulichung dient. 
Die Entwicklung der rms-Rauigkeit mit zunehmender Schichtdicke (Abbildung 5.23) zeigt noch einmal das bereits diskutierte leichte Aufrauen für die auf glattem Si deponierten PC-Schichten (rote Quadrate). Im Fall der PC-Schichten auf Wellenstrukturen (schwarze Quadrate) fällt die Rauigkeit zunächst exponentiell ab. Dies entspricht den theoretischen Erwartungen (Kapitel 3.5) und wurde auch schon bei C (Kapitel 5.2) beobachtet. Der Vorfaktor im Exponenten ist hier aber um einen Faktor 4 größer als bei C, so dass bereits bei einer Schichtdicke von etwa $70 \mathrm{~nm}$ die intrinsische Rauigkeit von PC-Schichten auf glatten Substraten erreicht wird. Diese Rauigkeiten können wie in der Theorie vorhergesagt nicht weiter geglättet werden.

Die spektrale Leistungsdichte der gewellten Si-Oberfläche wird ähnlich wie bei C mit zunehmender Menge PC frequenzabhängig geglättet (Abbildung 5.24), d.h. zunächst verschwinden die Maxima höherer Ordnung. Im Fall von PC ist bereits nach der Deposition von 4 nm PC (rote Kurve) das Maximum zweiter Ordnung verschwunden, erst bei $38 \mathrm{~nm}$ PC das erster Ordnung und schließlich bei etwas mehr als $63 \mathrm{~nm}$ auch das Hauptmaximum. Die erreichte Endkurve entspricht in etwa den Spektren, die auch für PC auf glattem Si beobachtet wurden. Auch hier ist ähnlich wie bei $\mathrm{C}$ ein frequenzunabhängiges Absinken der Spektren zu beobachten, das in Abschnitt 5.5 genauer diskutiert wird.

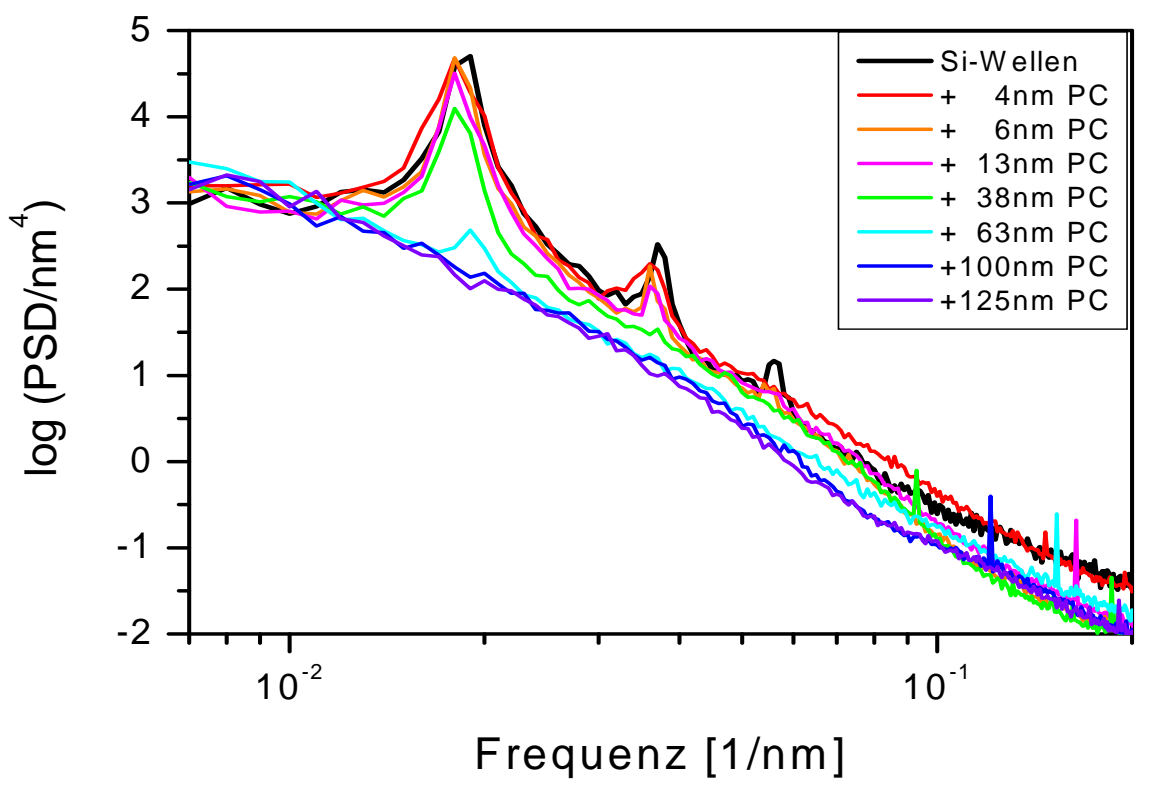

Abbildung 5.24: Veränderung der spektralen Leistungsdichte mit zunehmender Dicke der PC-Schicht. 
Insgesamt lässt sich sagen, dass sich PC sehr gut als Glättungsmaterial eignet: Im Vergleich zu allen bisher betrachteten Materialien glättet PC am Schnellsten, denn es sind nur $70 \mathrm{~nm}$ Schichtdicke vonnöten, um die Wellenstrukturen zu eliminieren. Die hinterlassene Endrauigkeit von 0,4 nm ist aufgrund der größeren Eigenrauigkeit etwas höher als bei PMMA $(0,3 \mathrm{~nm})$ und $C(0,2 \mathrm{~nm})$, dafür wird sie bereits bei kleinen Schichtdicken erreicht. Des Weiteren besteht die Oberfläche hauptsächlich aus sehr kleinen Hügelstrukturen von nur $20 \mathrm{~nm}$ bis $80 \mathrm{~nm}$ Größe. Dies kann für manche Anwendungen von Vorteil sein, z.B. wenn noch weitere Schichten aufgebracht werden sollen.

\subsection{Glättung durch $\mathrm{ZrO}_{2}$}

Als letztes Glättungsmaterial soll ein Oxid verwendet werden. In der Literatur findet man, dass $\mathrm{ZrO}_{2}$ bei Raumtemperatur sehr glatt wächst und sehr harte Filme bildet [Voev01]. Wie bei den anderen Materialien zuvor auch, werden zunächst wieder die Eigenschaften der Filme auf glatten Si(111)-Substraten untersucht. Zur Analyse des Wachstumsverhaltens von $\mathrm{ZrO}_{2}$ wurden Schichten mit Dicken zwischen $4 \mathrm{~nm}$ und $1100 \mathrm{~nm}$ deponiert und mittels Rasterkraftmikroskop charakterisiert. Abbildung 5.25 (a) zeigt beispielhaft die AFM-Aufnahme einer $605 \mathrm{~nm}$ dicken Schicht, die eine rms-Rauigkeit von lediglich 0,08 nm hat. Analysiert man die Oberflächenrauigkeit in Abhängigkeit von der Schichtdicke für eine Bildgröße von $1 \mathrm{x} 1 \mu \mathrm{m}^{2}$, so erhält man den in Abbildung 5.25 (b) dargestellten Verlauf. Es ist bis zu einer Schichtdicke von 1,1 $\mu \mathrm{m}$ kein Anstieg der Rauigkeit beobachtbar und die mittlere rms-Rauigkeit des $\mathrm{ZrO}_{2}$ liegt konstant etwas unter 0,1 nm. Aufgrund dieser ungewöhnlich niedrigen Rauigkeiten wurden die Proben noch etwas genauer analysiert. Über Reflektometriemessungen und deren Simulation mittels des Programms IMD konnten die Schichtdicken und Oberflächen- bzw. Grenzflächenrauigkeiten der dünneren Filme bestimmt werden. Beispielhaft sind vier Graphen mit dazugehörigen Simulationen in Abbildung 5.25 (c) dargestellt. Auf diese Weise wurde eine Depositionsrate von 0,025 nm/Puls bestimmt, die während des gesamten 
Herstellungsprozesses konstant blieb. Die Oberflächenrauigkeit bestimmt aus den Reflektometriemessungen liegt bei etwa $1 \mathrm{~nm}$ unabhängig von der Schichtdicke.
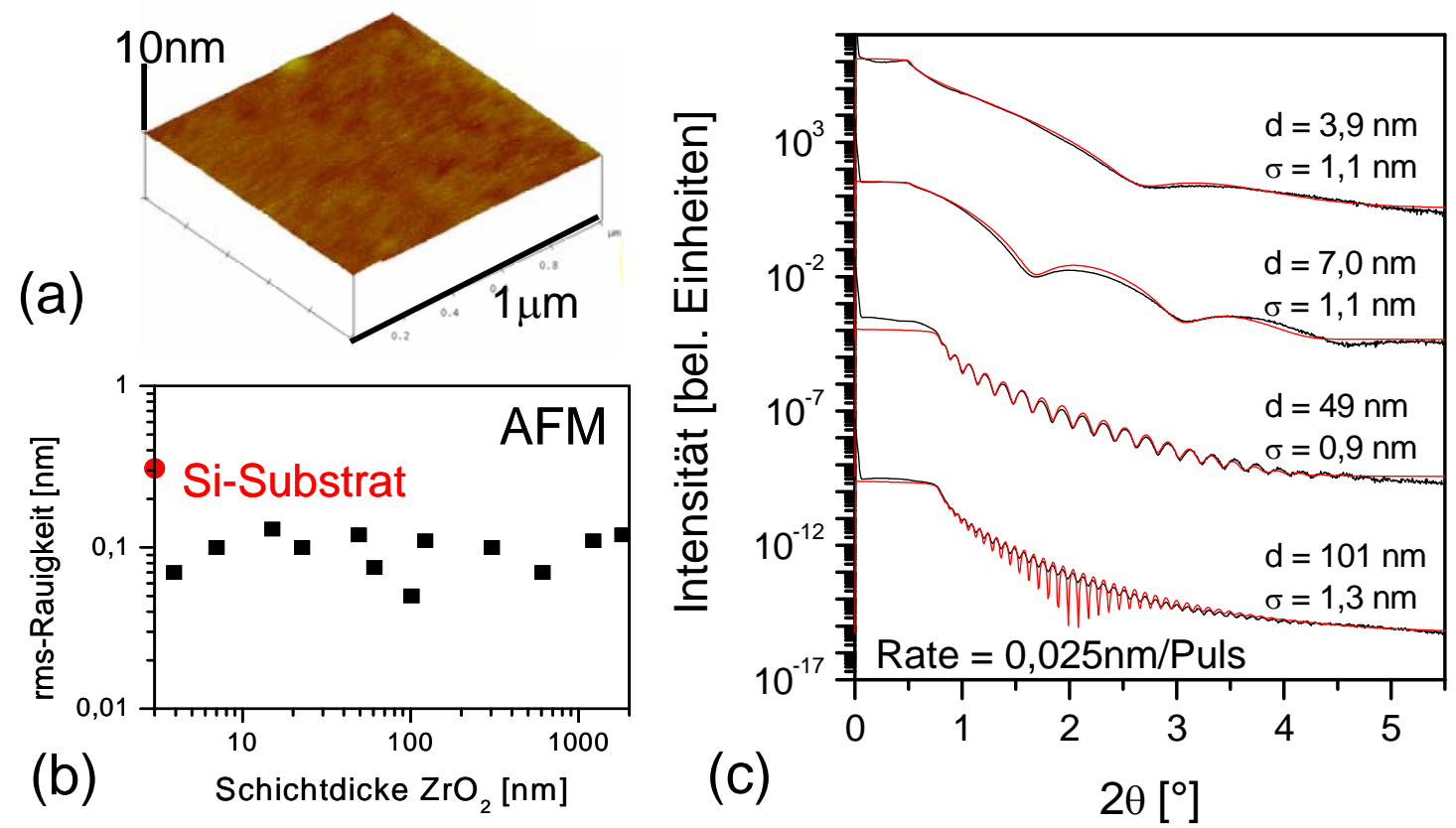

Abbildung 5.25: (a) zeigt eine AFM-Detailaufnahme eines 605 nm dicken Films. In (b) ist der Verlauf der mittels AFM gemessenen Rauigkeit mit der Schichtdicke aufgetragen. (c) zeigt XRR-Messungen mit dazugehörigen Simulationen zur Raten- und Rauigkeitsbestimmung.

Diese aus den XRR-Messungen bestimmten Rauigkeiten weichen um etwa eine Größenordnung von den durch AFM ermittelten Werten ab. Dies ist ein bekanntes Phänomen und darauf zurückzuführen, dass bei der Reflektometrie über eine makroskopische Fläche gemittelt wird und somit langwellige Rauigkeiten aufgrund von Inhomogenitäten der Schichtdicke oder leichte Krümmungen des Substrates mitgemessen werden [Li03]. Mit dem AFM dagegen bestimmt man die lokale Rauigkeit vergleichsweise kleiner Bildausschnitte (typischerweise zwischen $1 \mathrm{x} 1 \mu \mathrm{m}^{2}$ und 10x10 $\mu \mathrm{m}^{2}$ ), die für diese Arbeit von Interesse sind. Interessanterweise wird selbst das bereits relativ glatte SiSubstrat $\left(\sigma_{r m s}=0,3 \mathrm{~nm}\right)$ schon bei der dünnsten deponierten $\mathrm{ZrO}_{2}$-Schichtdicke noch weiter ausgeglättet.

Die spektrale Leistungsdichte einer dicken $\mathrm{ZrO}_{2}$-Schicht ist in Abbildung 5.26 gezeigt. Analog zum Vorgehen bei den C und PC-Schichten wurde auch hier das Spektrum 
durch einen Fit mit Gleichung 3.13 unter Vernachlässigung der Mechanismen der Ordnung 1 und 3 (vergleiche Kapitel 3.3) analysiert, um die relevanten Wachstumsmechanismen für dieses Material ermitteln. Ein Vergleich der Koeffizienten $b_{i}$ (wie in Kapitel 3.2 beschrieben) zeigt, dass bis zu einer kritischen Strukturgröße von etwa $110 \mathrm{~nm}$ ein Mechanismus der Ordnung $i=4$ die Glättung bestimmt, während für größere Strukturen ein Mechanismus der Ordnung $i=2$ dominiert. Somit ist Diffusion für die Glättung kleiner Strukturen verantwortlich [Herr50] und entweder ballistischer Transport [Mose05] oder Sputtererosion [Brad88] für die Abnahme größerer Strukturen.

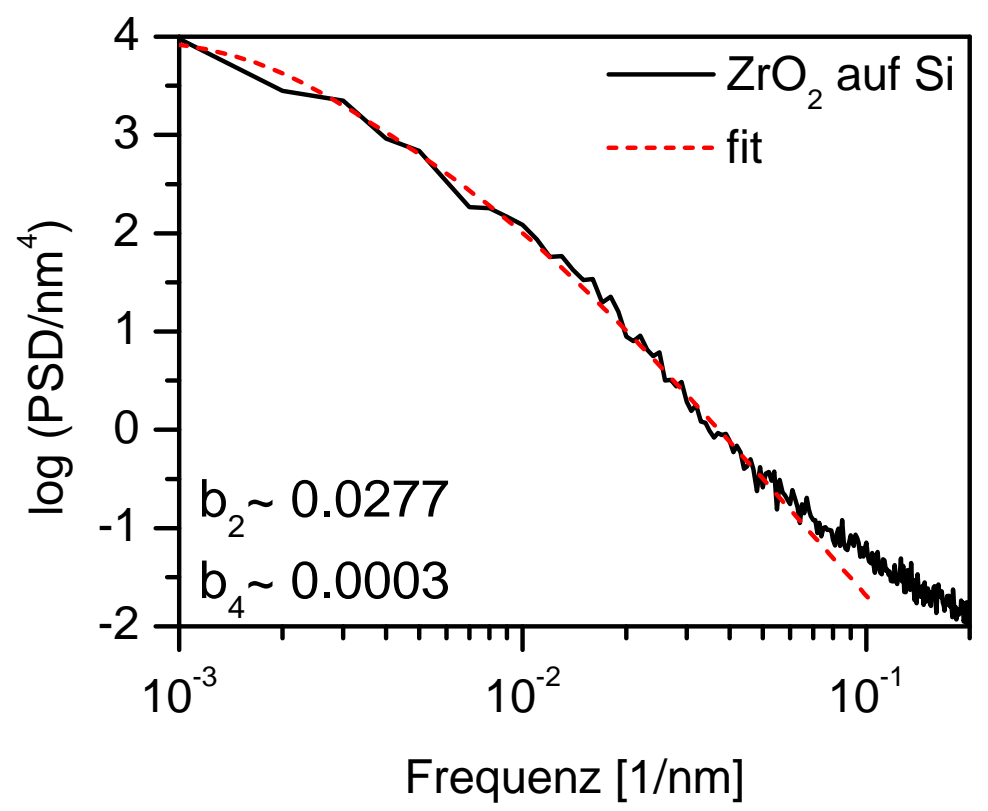

Abbildung 5.26: Eine typische spektrale Leistungsdichte eines $\mathrm{ZrO}_{2}$-Films auf glattem Si mit Fit zur Bestimmung der Glättungsmechanismen.

Es hat sich also gezeigt, dass $\mathrm{ZrO}_{2}$ mit einer sehr geringen intrinsischen rmsRauigkeit von nur 0,1 nm auf glatten Si-Substraten aufwächst. Dies ist weniger als bei allen anderen bisher untersuchten Systemen. Auch hier wird im Folgenden wird das Glättungsverhalten von $\mathrm{ZrO}_{2}$ auf Substraten mit Wellenstrukturen untersucht. In Abbildung 5.27 sind rasterkraftmikroskopische Bilder dieser Strukturen vor und nach der Beschichtung mit den jeweils angegebenen Mengen $\mathrm{ZrO}_{2} \mathrm{zu}$ sehen. Es lässt sich gut beobachten, dass die Höhe der Wellen ähnlich wie schon bei C und PC beobachtet mit zunehmender Menge $\mathrm{ZrO}_{2}$ abnimmt, was der sukzessiven Glättung dieser Frequenz entspricht. Vergleicht man die Aufnahmen (a) bis (c), so werden die beschichteten 
Wellenkämme deutlich regelmäßiger und glatter im Vergleich zu den ursprünglichen Strukturen. Dies lässt auch hier wieder auf eine frequenzabhängige Oberflächenveränderung schließen, die bewirkt, dass kleine Strukturen zuerst ausgeglättet werden. Die Bilder zeigen, dass etwas mehr als $200 \mathrm{~nm}$ Material benötigt wird, um die ursprüngliche Wellenfrequenz vollständig zu glätten. Dagegen bleiben langwelligere Strukturen wie in Abbildung 5.27(f) auch nach der Deposition von $500 \mathrm{~nm} \mathrm{ZrO}_{2}$ noch bestehen.
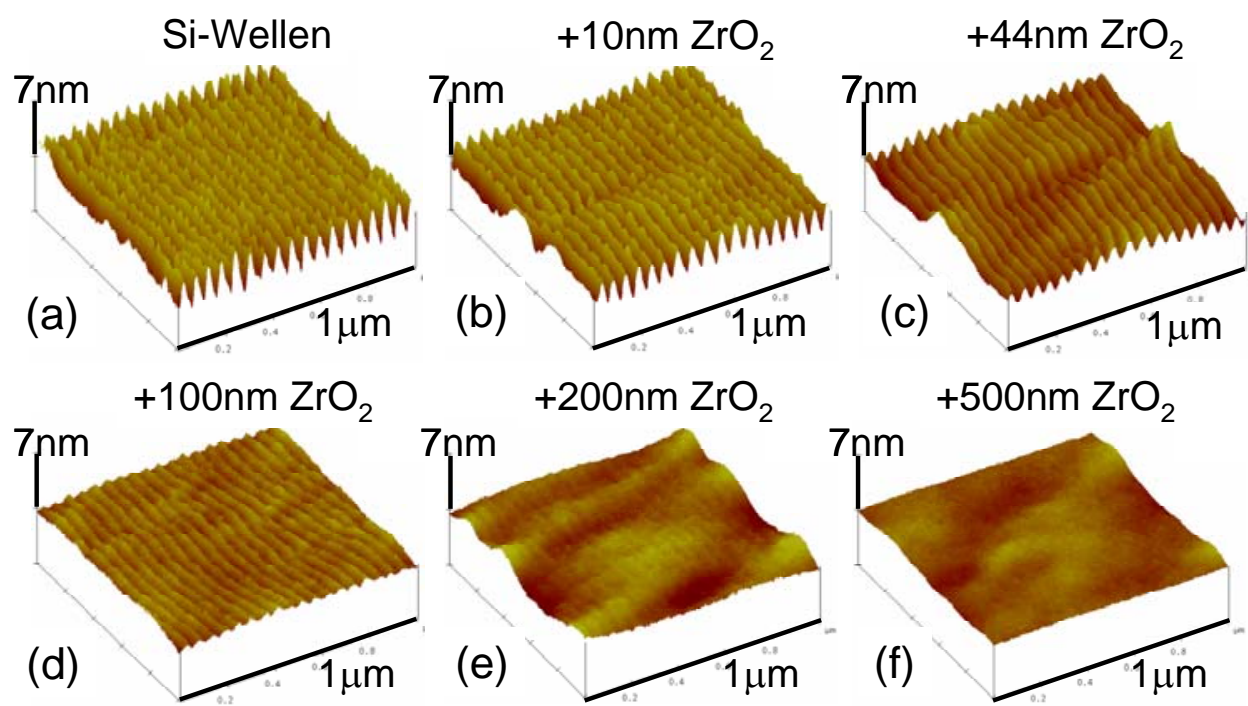

Abbildung 5.27: AFM-Aufnahmen der gewellten Substrate vor (a) und nach (b)-(f) der Beschichtung mit $\mathrm{ZrO}_{2}$-Dicken zwischen $10 \mathrm{~nm}$ und $500 \mathrm{~nm}$. Die Oberfläche wird sukzessive geglättet, wobei die verwendeten Schichtdicken nicht ausreichen, Strukturen großer Wellenlänge auszuglätten (siehe (e) und (f)).
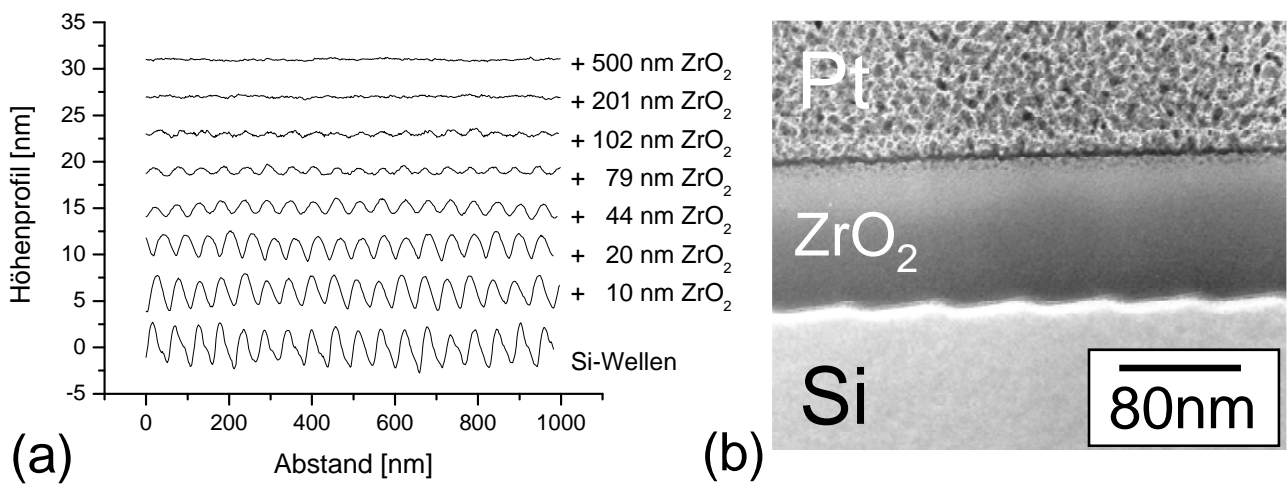

(b)

$80 \mathrm{~nm}$

Abbildung 5.28: (a) Höhenprofile (übereinander angeordnet) der mit unterschiedlicher Menge $\mathrm{ZrO}_{2}$ geglätteten Si-Wellenstrukturen. (b) TEMQuerschnittsaufnahme einer mit 102 nm $\mathrm{ZrO}_{2}$ geglätteten Probe. 
Dasselbe Ergebnis liefert der Vergleich der Höhenprofile einzelner Proben, die in Abbildung 5.28(a) dargestellt sind. Mithilfe der TEM-Querschnittsaufnahmen einer mit $100 \mathrm{~nm} \mathrm{ZrO}_{2}$ bedeckten Probe (Abbildung 5.28(b)) lassen sich die inneren Grenzflächen nach Deposition des Glättungsmaterials beobachten. Man sieht, dass die ursprüngliche Oberfläche während der Deposition nicht zerstört wurde.

Die Analyse der rms-Rauigkeiten eines $1 \mathrm{x} 1 \mu \mathrm{m}^{2}$ großen Bildausschnitt zeigt, dass auch hier die Oberfläche zunächst exponentiell mit der Schichtdicke glatter wird (vergleiche Abbildung 5.29(a)). Wie bereits bei C und PC diskutiert, entspricht dieses Verhalten den theoretischen Erwartungen (Abschnitt 3.5). Der Vorfaktor des Exponenten ist etwa genauso groß wie bei PC und damit wieder um einen Faktor 4 größer als bei C. Ab einer Schichtdicke von $100 \mathrm{~nm}$ bleibt die Rauigkeit bei etwa 0,3 nm konstant (vergleiche Abbildung 5.29(b)). Dieser Wert liegt etwas höher als die durchschnittliche Rauigkeit von 0,1 nm, die $\mathrm{ZrO}_{2}$ auf glattem $\mathrm{Si}$ aufweist. Ursache hierfür ist die bereits oben und in Kapitel 4.1 beschriebene langwellige Überstruktur der Wellensubstrate, die mit den hier verwendeten Schichtdicken noch nicht geglättet werden konnte.
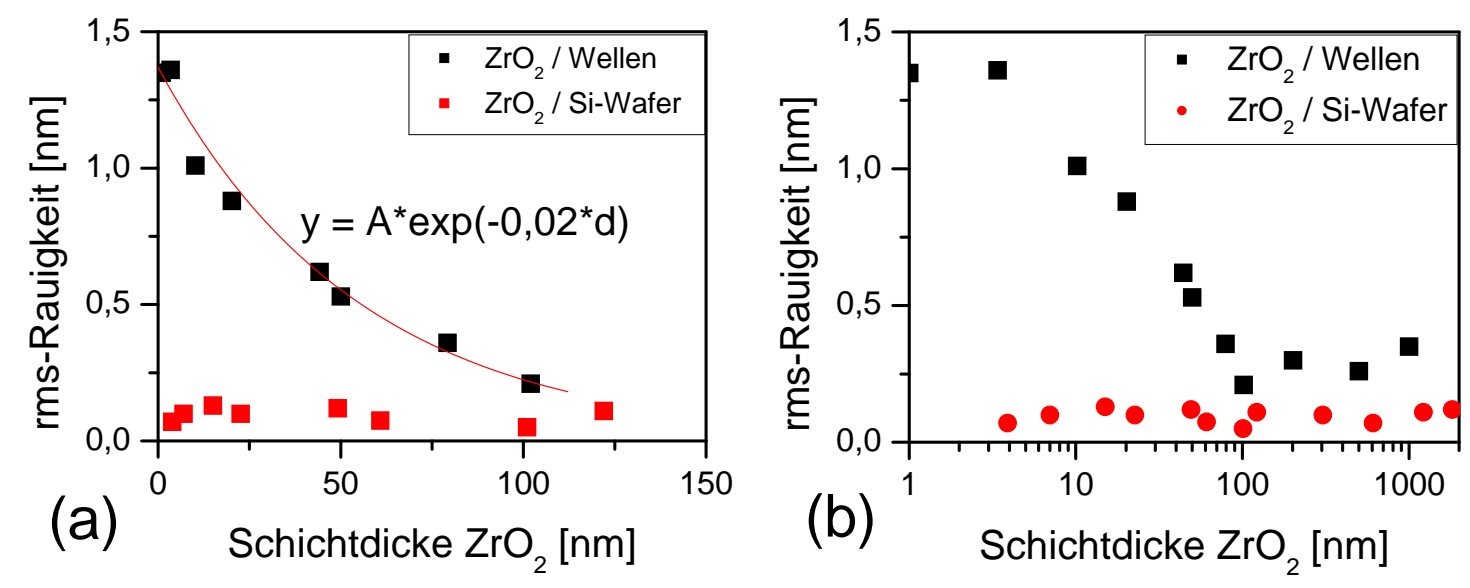

Abbildung 5.29: Entwicklung der rms-Rauigkeit mit der deponierten $\mathrm{ZrO}_{2}$ Schichtdicke. Nach einem exponentiellen Abfall der Rauigkeit bis etwa $100 \mathrm{~nm}$ Schichtdicke (siehe (a)), bleibt die Rauigkeit konstant bei einem Wert von etwa $0,3 \mathrm{~nm}$ (vergleiche (b)).

Abbildung 5.30 zeigt die Veränderung der spektralen Leistungsdichte der gewellten Si-Oberfläche mit zunehmender Menge $\mathrm{ZrO}_{2}$. Auch hier wird das ursprüngliche Spektrum 
wieder sukzessive von höheren zu niedrigeren Frequenzen hin geglättet. Als Beispiel sei die grüne Kurve angeführt, die einer $\mathrm{ZrO}_{2}$-Schichtdicke von $79 \mathrm{~nm}$ entspricht. Hier ist das vierte Maximum bereits komplett verschwunden, das dritte nur noch sehr schwach vorhanden, während die ersten beiden noch deutlich erkennbar sind. Bei einer Schichtdicke von etwa $200 \mathrm{~nm}$ ist die Hauptfrequenz der Wellenstruktur von $55 \mathrm{~nm}$ so gut wie vollständig geglättet. Ab hier dominiert wie in der Theorie beschrieben der Beitrag der aufwachsenden Schicht die Rauigkeit des gemessenen Frequenzbereichs, also der Rauschterm, der in Gleichung 3.13 dargestellt ist. Das Spektrum der mit $500 \mathrm{~nm} \mathrm{ZrO}_{2}$ bedeckten Oberfläche wurde mit dieser Gleichung gefittet. Das Ergebnis ist als Inset in Abbildung 5.30 dargestellt, die entsprechenden $b_{\mathrm{i}}$-Werte sind angegeben. Sie deuten darauf hin, dass Strukturgrößen bis $(70 \pm 7) \mathrm{nm}$ hauptsächlich durch Diffusion geglättet werden [Herr50], darüber gewinnen Glättungsmechanismen wie ballistischer Transport [Mose05] oder Sputtererosion [Brad88] an Bedeutung. Neben dieser frequenzabhängigen Glättung beobachtet man ähnlich wie bei C und PC auch hier mit zunehmender Schichtdicke ein deutliches Absinken der Kurven über fast den kompletten Frequenzbereich.

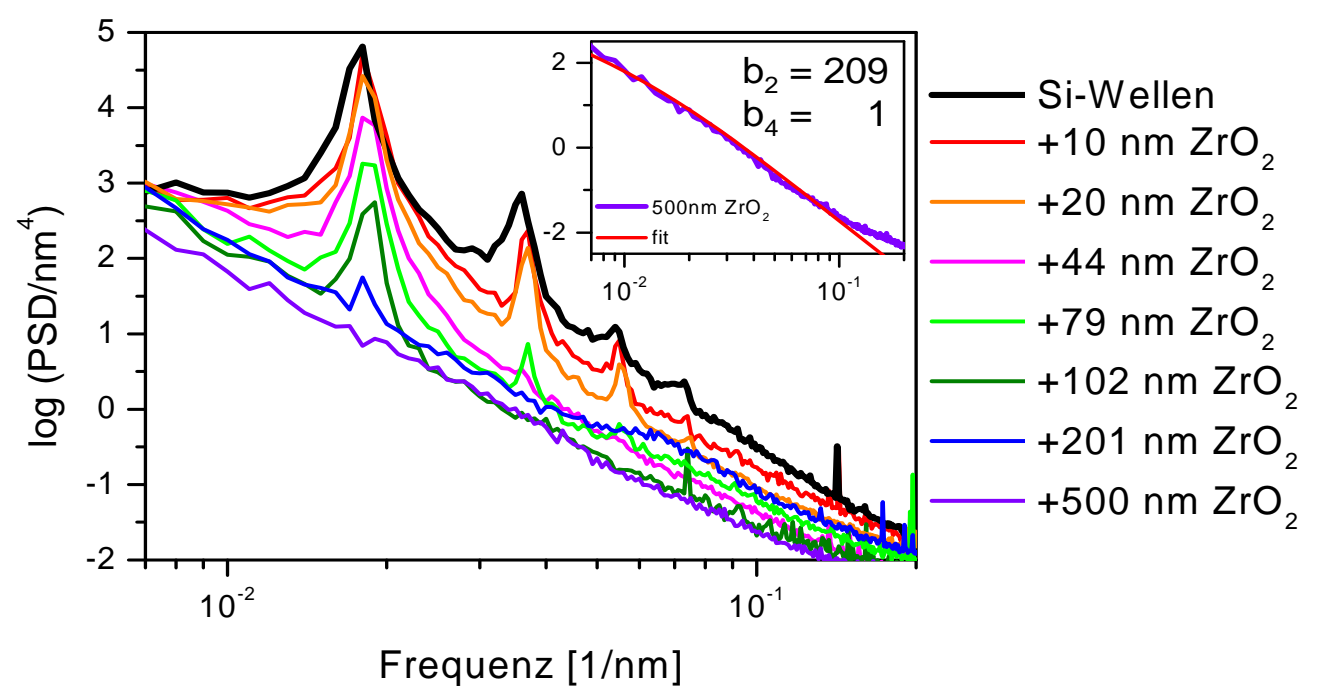

Abbildung 5.30: Veränderung der spektralen Leistungsdichte mit zunehmender Schichtdicke. Neben einem Absinken der Spektren beobachtet man, dass die Maxima von rechts nach links also von hohen zu tiefen Frequenzen geglättet werden. Als Inset ist der Fit des Spektrums der mit $500 \mathrm{~nm} \mathrm{ZrO}_{2}$ bedeckten Oberfläche angegeben. Hierfür wurde Gleichung 3.13 benutzt, da dieser Term den geglätteten Bereich der Spektren dominiert. 
Um die Vorgänge bei der Glättung genauer zu analysieren, sollen die Spektren mit Hilfe von Gleichung 3.14 theoretisch simuliert werden. $\mathrm{ZrO}_{2}$ eignet sich hierfür als Beispielmaterial, da es eine sehr geringe Endrauigkeit erzeugt und sich die spektrale Leistungsdichte der Wellenoberfläche von der der geglätteten Oberfläche deutlich unterscheidet. Als Anfangsspektrum wurde die gemessene spektrale Leistungsdichte eines gewellten Si-Substrats eingesetzt. Die freien Parameter $R, b_{2}$ und $b_{4}$ der Gleichung wurden mithilfe des Fits aus Abbildung 5.30 so gewählt, dass das gemessene Endspektrum nach erfolgreicher Glättung erreicht wird. Man erhält auf diese Weise den in Abbildung 5.31 dargestellten Verlauf der spektralen Leistungsdichten. Die gemessenen Anfangs- und Endkurven sind ebenfalls in Abbildung 5.31 dargestellt.

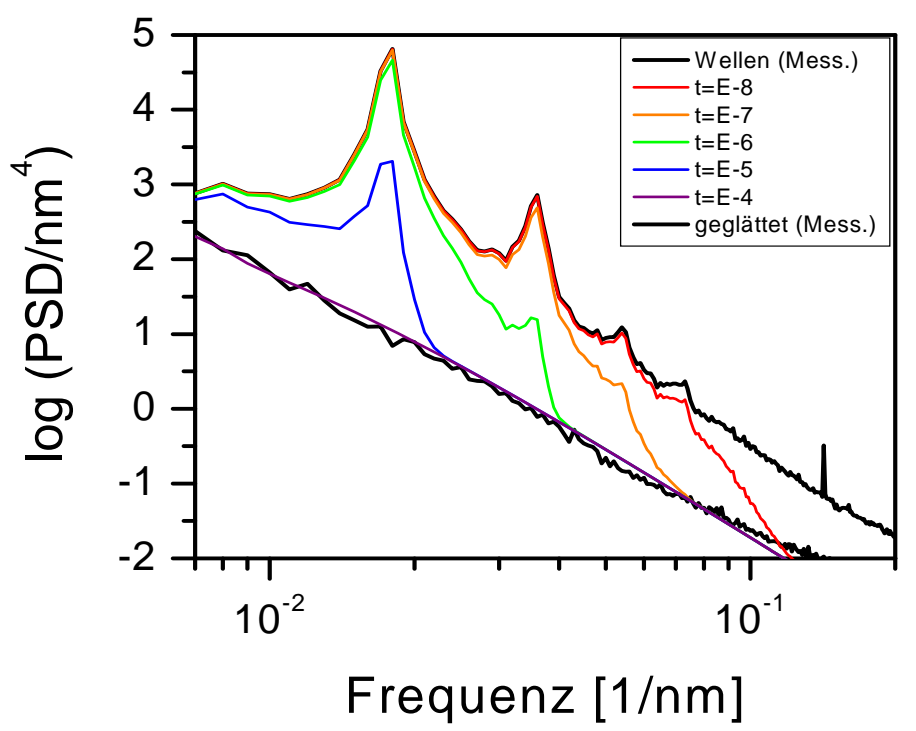

\begin{abstract}
Abbildung 5.31: Simulation der spektralen Leistungsdichte. Die freien Parameter wurden mithilfe der komplett geglätteten Messkurve angepasst (schwarz dargestellt). Da bei den gemessenen Spektren (Abbildung 5.30) keine Abknickpunkte abgeschätzt werden können, ist eine Zuordnung der t-Werte zu realen Schichtdicken nicht möglich.
\end{abstract}

Mithilfe dieser Simulationen lässt sich der frequenzabhängige Anteil der Glättung gut nachvollziehen: Mit zunehmender Schichtdicke (d.h. anwachsender Depositionszeit $t$ ) sinkt der hochfrequente Teil der spektralen Leistungsdichte immer weiter ab und wird wie erwartet überlagert von den durch Rauschen erzeugten Rauigkeiten der anwachsenden Schicht. Im Unterschied zu den Simulationen sinken die gemessenen spektralen 
Leistungsdichten sukzessive weiter ab, obwohl der Glättungsbereich zumindest bei den hohen Frequenzen schon durchgelaufen ist. Eine Erklärung dafür ist schwierig zu finden. Als mögliche Ursache lässt sich vermuten, dass entweder das lineare Modell hier zur vollständigen Beschreibung nicht ausreicht oder eine Schichtdickenabhängigkeit der $b_{\mathrm{i}^{-}}$ Werte vorliegt.

Die bei der Simulation deutlich sichtbaren (und erwarteten) Abknickpunkte in den Spektren (vergleiche Abbildung 3.4) sind bei den experimentell bestimmten spektralen Leistungsdichten nur erahnbar, da sie von den dominanten Frequenzen der Wellenstruktur überlagert werden. Es wurde daher hier auf eine konkrete Bestimmung verzichtet. Dies führt dazu, dass den $t$-Werten in den Simulationen keine realen Schichtdicken zugeordnet werden können.

Zusammenfassend hat $\mathrm{ZrO}_{2}$ mit nur $0,1 \mathrm{~nm}$ die geringste Eigenrauigkeit aller vorgestellten Glättungsmaterialien. Damit erfüllt es das erste der zwei geforderten Kriterien. Des Weiteren glättet es die auf Si-Substraten vorhandenen Rauigkeiten sehr schnell: Der exponentielle Abfall ist deutlich schneller als bei C und ähnlich schnell wie bei PC. Die benötigte kritische Schichtdicke von etwas mehr als $100 \mathrm{~nm}$ ist etwas höher als bei PC (70 nm), was daran liegt, dass die Endrauigkeit deutlich geringere Werte annimmt. Die Simulation des Glättungsverlaufs hat gezeigt, dass der frequenzabhängige Teil der Glättung von der Theorie gut beschrieben wird, nicht aber das beobachtete frequenzunabhängige Absinken der Kurven. Für eine genaue Analyse und einen direkten Vergleich mit der Theorie erwiesen sich die dominanten Frequenzen der Wellenstrukturen als störend. Daher soll in Kapitel 6 die Glättung anhand von statistisch rauen Oberflächen untersucht werden, deren spektralen Leistungsdichten gleichmäßig abfallen. Zunächst werden die verwendeten Materialien aber noch einmal vergleichend dargestellt.

\subsection{Vergleich}

Als Voraussetzung für ein zur effizienten Glättung geeignetes Material müssen aufgrund der Theorie zwei Kriterien erfüllt sein: Es muss eine geringe intrinsische 
Rauigkeit beim Wachstum auf glatten Substraten besitzen und vorhandene Oberflächenrauigkeiten schnell ausglätten.

Als Vertreter für kristalline Materialien wurde die Glättung zunächst mit Ag untersucht. Aufgrund des kristallinen Inselwachstums und der starken Diffusion ist die intrinsische Rauigkeit von Ag mit 0,8 nm und mehr sehr hoch. Dadurch eignet sich Ag nicht gut zum Glätten. Die Si-Wellenstrukturen werden zwar schon nach der Deposition von $8 \mathrm{~nm}$ Material von den Ag-Inseln überlagert, allerdings sind die neu erzeugten Rauigkeiten von 1,4 nm nicht geringer als die ursprünglichen. Vielmehr wird die Wellenstruktur der Oberfläche nur in eine statistische Inselstruktur umgewandelt.

Die anderen vier verwendeten Materialien zeichneten sich durch eine sehr geringe intrinsische Rauigkeit aus und erfüllten damit eine wichtige Voraussetzung für Glättungsmaterialien. Für eine effiziente Glättung ist weiterhin eine schnelle Verringerung der vorgegebenen Oberflächenrauigkeit notwendig. Als Parameter dafür diente die Schichtdicke, die notwendig war, um eine Wellenstruktur mit 55 nm Periodizität und einer Rauigkeit von etwa 1,3 nm auszuglätten. Im Fall von C mit einer Eigenrauigkeit von nur 0,2 $\mathrm{nm}$ hat sich gezeigt, dass hierfür Schichtdicken von weit über $230 \mathrm{~nm}$ notwendig wären, die aufgrund der schlechten Haftungseigenschaften auf Si und großen Spannungen im Film nicht erreicht werden konnten. Für Si als Substratmaterial und möglicherweise auch PLD als Depositionsmethode eignet sich dieses Material folglich nicht zur Glättung. Dies war anders im Fall von PC und $\mathrm{ZrO}_{2}$, die die Si-Wellen ähnlich kontinuierlich glätten konnten wie C. Hier waren allerdings deutlich geringere Schichtdicken notwendig, um eine vollständige Glättung zu erzielen. Zum besseren Vergleich sind in Abbildung 5.32 die bereits einzeln diskutierten Verläufe der Rauigkeiten mit steigender deponierter Schichtdicke für die Materialien $\mathrm{ZrO}_{2}, \mathrm{C}$ und PC in einem Graph dargestellt. Die Dreiecke stellen jeweils die Rauigkeit auf glattem Si dar, die Vierecke auf den Wellenstrukturen. Es zeigt sich anhand des gefitteten exponentiellen Abfalls (die Gleichungen sind jeweils angegeben), dass die Glättung im Fall von $\mathrm{C}$ wesentlich langsamer abläuft als für $\mathrm{ZrO}_{2}$ und PC, obwohl alle drei Materialien eine sehr geringe intrinsische Rauigkeit auf glatten Substraten haben. Im direkten Vergleich von PC und $\mathrm{ZrO}_{2}$ stellt sich $\mathrm{ZrO}_{2}$ als das etwas bessere Glättungsmaterial dar, da geringere Endrauigkeiten erreicht werden können, auch wenn dafür etwas mehr Material deponiert werden muss. 


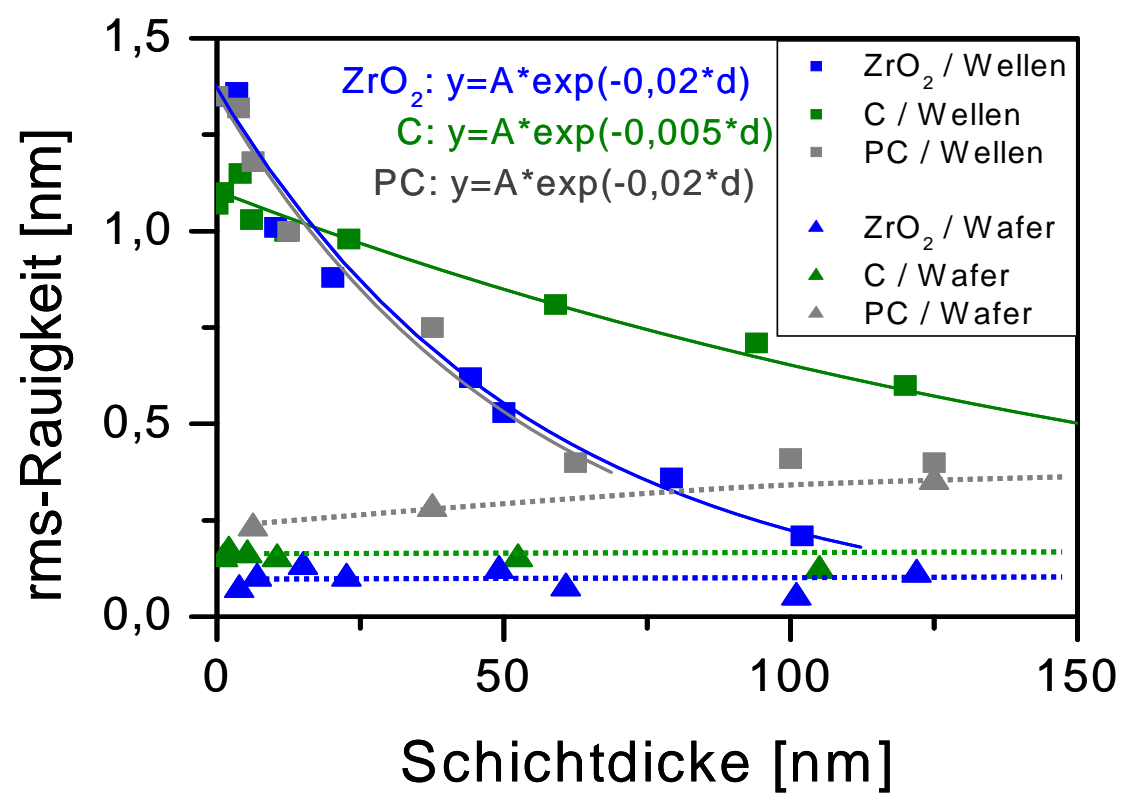

Abbildung 5.32: Vergleich der Rauigkeitsverläufe für die Materialien $\mathrm{ZrO}_{2}, \mathrm{C}$ und PC. Die Dreiecke beschreiben die Rauigkeiten auf glatten Substraten, die Quadrate auf den Si-Wellenstrukturen. Im Fall der C-Proben hatten die gewellten Substrate eine geringere Anfangsrauigkeit von nur etwa 1,1 nm im Gegensatz zu 1,3 nm bei den anderen beiden Materialien. Die exponentiellen Abfälle wurden gefittet und bei den Filmen auf glatten Substraten wurden zur Veranschaulichung Hilfslinien eingezeichnet.

PMMA ist ebenfalls ein sehr gutes Glättungsmaterial. Obwohl die starke Entnetzung in den Anfangsstadien des Wachstums zu einer starken Aufrauung bei dünnen deponierten Schichten führt, sind trotzdem nur etwa $200 \mathrm{~nm}$ Schichtdicke notwendig, um die Oberfläche komplett zu glätten. Die Endrauigkeit beträgt dabei 0,3 nm. Der Nachteil ist, dass sich mit PMMA die Anfangsstadien der Glättung einer rauen Oberfläche schlecht beobachten lassen, da die Effekte überlagert werden von der Entnetzung. Daher soll im Folgenden $\mathrm{ZrO}_{2}$ als Glättungsmaterial verwendet werden, um die einzelnen Glättungsstadien im Detail spektral aufgelöst zu untersuchen. Hierfür ist es sinnvoll, statistisch raue Oberflächen als Ausgangsmaterial zu nehmen, da die dazugehörigen spektralen Leistungsdichten einen gleichmäßigen Verlauf zeigen, bei dem sich Veränderungen gut beobachten lassen. 


\section{Frequenzabhängige Glättung statistischer Oberflächenstrukturen}

Im vorherigen Kapitel hat sich gezeigt, dass sich $\mathrm{ZrO}_{2}$ von allen betrachteten Materialien am besten zur Untersuchung der Glättung rauer Oberflächen eignet. Des Weiteren ist es sinnvoll, eine statistisch raue Oberfläche zu verwenden, wie sie beispielsweise beim Wachstum von Ag auf Si auftritt (vergleiche Kapitel 4.2). Hierbei können Veränderungen in der spektralen Leistungsdichte besser beobachtet werden. Ein weiterer Vorteil der Untersuchung solcher Oberflächen ist, dass die meisten rauen Oberflächen, wie sie beispielsweise bei der Deposition dünner Schichten entstehen, keinerlei räumliche Vorzugsorientierung aufweisen. Sie kommen daher in der Anwendung deutlich häufiger vor. Im Folgenden werden die unterschiedlichen Zwischenschritte bis zur vollständigen Glättung spektral aufgelöst untersucht, die bei der Glättung vorherrschenden Mechanismen analysiert und die Vorgänge bei der Glättung simuliert (Kapitel 6.1). In Abschnitt 6.2 soll die mittlere Insel-/ Korngröße der rauen Ag-Unterlage systematisch variiert und der Glättungsprozess abhängig von der vorherrschenden Strukturgröße auf dem Substrat untersucht werden.

\subsection{Schichtdickenabhängigkeit der Glättung}

Zur detaillierten Untersuchung der Glättungsvorgänge wurden Ag-Schichten einer Dicke von $44 \mathrm{~nm}$ als Substrate ausgewählt (siehe Abbildung 6.1(a)), da sie mehrere 
wichtige Anforderungen erfüllen: Die Ag-Schichten sind vollständig geschlossen und ihre Anfangsrauigkeit von 0,8 nm lässt sich reproduzierbar einstellen, da sie sich auch bei leichten Schichtdickenschwankungen kaum ändert. Des Weiteren zeigt die spektrale Leistungsdichte einen sehr gleichmäßigen Abfall mit einer Steigung von -5,7 (Abbildung 6.1(b)), so dass sich Veränderungen aufgrund der Glättungsmechanismen bei unterschiedlichen Frequenzen gut registrieren lassen. Die einzige charakteristische Strukturgröße auf der Oberfläche rührt von der lateralen Korngröße her und beträgt etwa $(86 \pm 5) \mathrm{nm}$. Dies entspricht einer Frequenz von etwa $1,2 \cdot 10^{-2} \mathrm{~nm}^{-1}$ und liegt damit innerhalb des untersuchten Spektralbereichs. Die verwendeten Substrate wurden wie in Kapitel 4.2 beschrieben hergestellt.
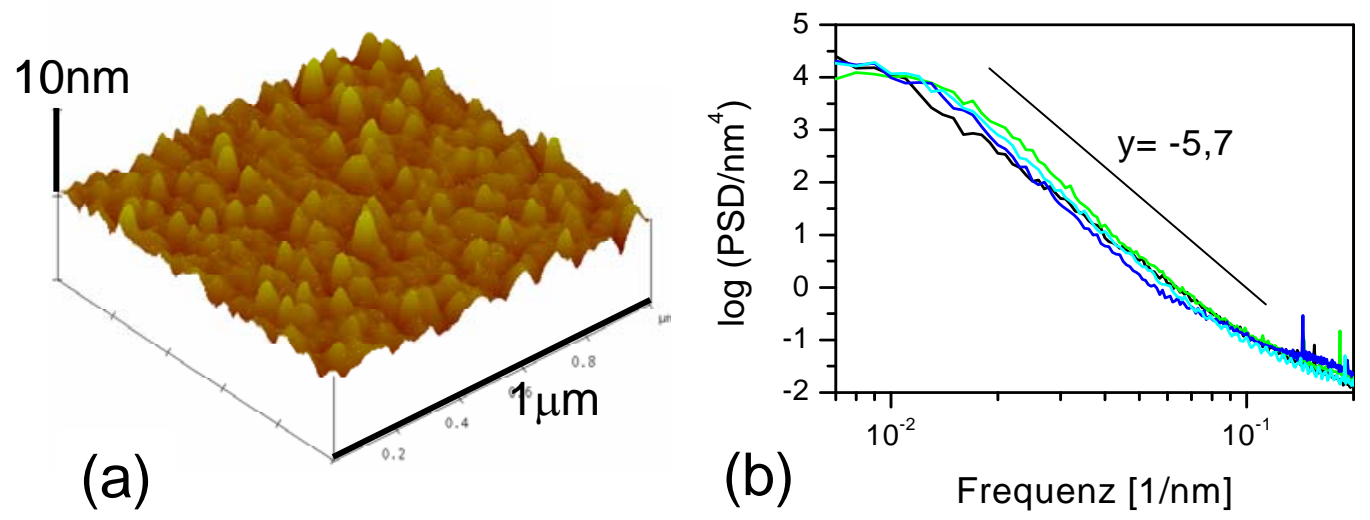

\begin{abstract}
Abbildung 6.1: (a) AFM-Bild einer $44 \mathrm{~nm}$ dicken Ag-Schicht, wie sie als Substrat für die Glättungsexperimente verwendet wird. (b) Die spektralen Leistungsdichten verschiedener solcher Ag-Schichten (gleiche Herstellungsbedingungen) zeigen die gute Reproduzierbarkeit.
\end{abstract}

Deponiert man auf diese Oberflächen unterschiedliche Mengen $\mathrm{ZrO}_{2}$, so werden die vorhandenen Strukturen der Ag-Oberflächen mit zunehmender Schichtdicke sukzessive ausgeglättet. Die Änderung der Morphologie lässt sich in Abbildung 6.2 anhand von sechs AFM-Bildern für $\mathrm{ZrO}_{2}$-Schichtdicken zwischen $2 \mathrm{~nm}$ und $370 \mathrm{~nm}$ verfolgen. Zur besseren Vergleichbarkeit sind in Abbildung 6.3 charakteristische Höhenprofile unterschiedlich dicker $\mathrm{ZrO}_{2}$-Schichten auf den $44 \mathrm{~nm}$ dicken Ag-Schichten dargestellt. Neben der Abflachung des Höhenprofils ist in allen Abbildungen eine laterale Verbreiterung der Inseln zu beobachten. Weiterhin werden scharfe Kanten und kleine Strukturen, die im Fourierspektrum durch hohe Frequenzen dargestellt werden, als erstes ausgeglättet. Für 
langwelligere Strukturen auf der Oberfläche dagegen sind deutlich größere Schichtdicken notwendig und selbst nach der Deposition von etwa $1000 \mathrm{~nm} \mathrm{ZrO}_{2}$ kann keine komplette Glättung erzielt werden (vergleiche die Höhenprofile).

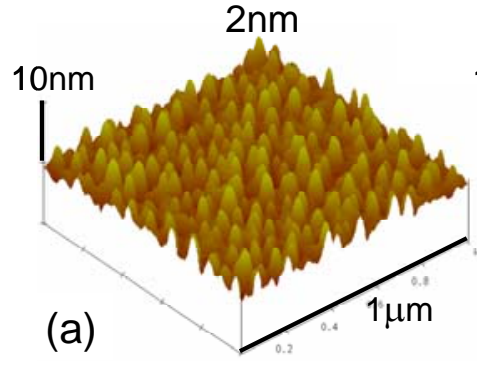

$85 \mathrm{~nm}$

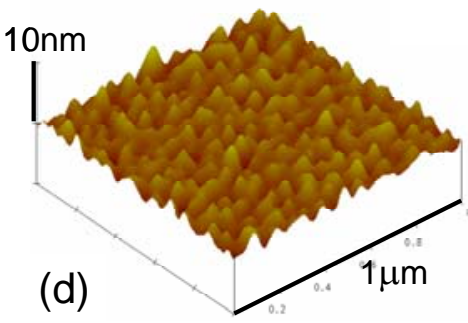

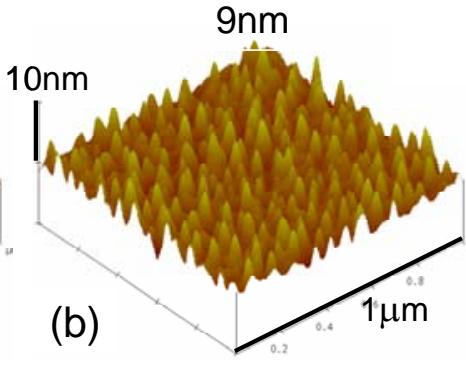

170nm

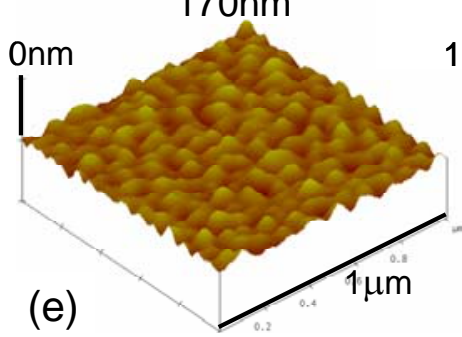

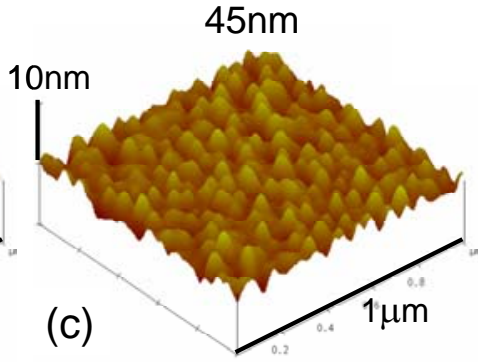

$370 \mathrm{~nm}$

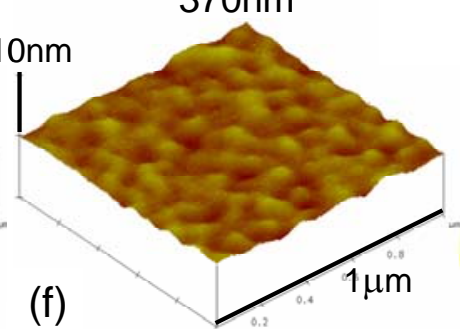

Abbildung 6.2: (a)-(f) Die AFM-Bilder zeigen die Veränderungen der Oberflächen von $44 \mathrm{~nm}$ dicken Ag-Filmen, auf denen die angegebenen Mengen $\mathrm{ZrO}_{2}(2 \mathrm{~nm}$ bis $370 \mathrm{~nm})$ deponiert wurden.

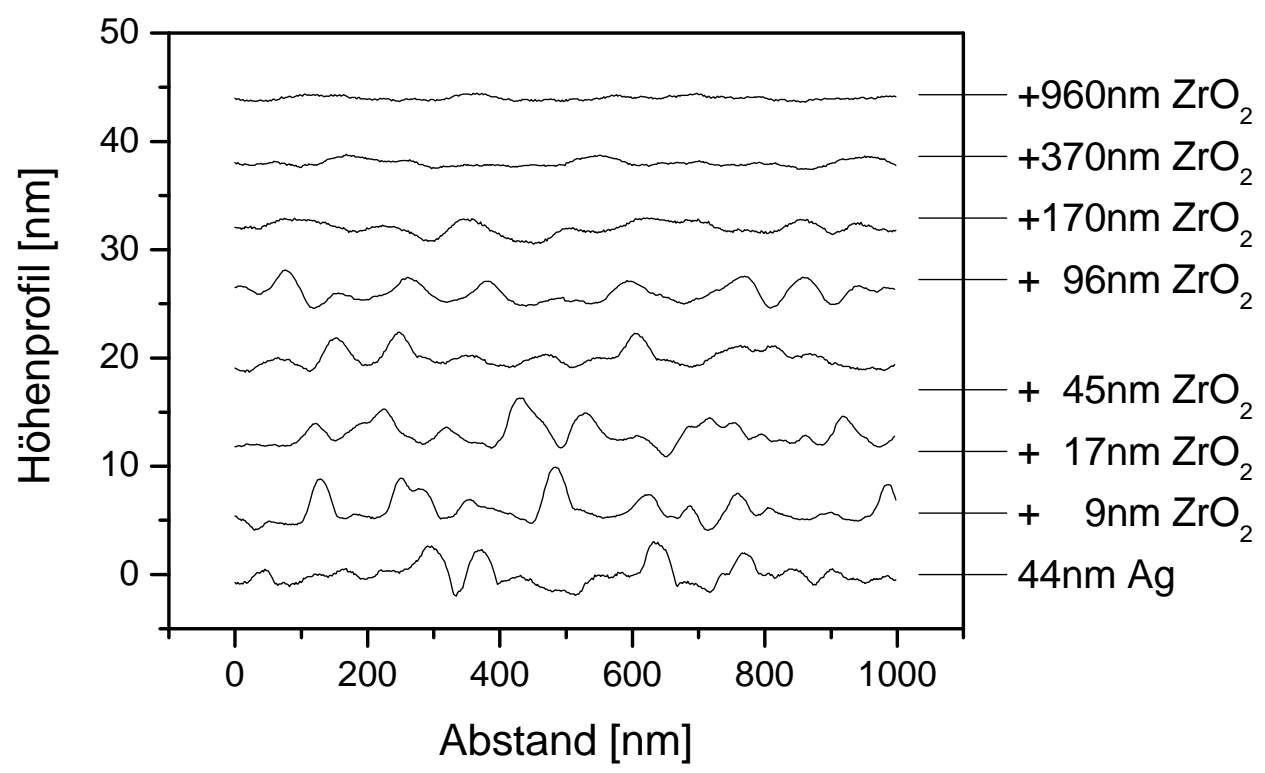

Abbildung 6.3: Übereinander angeordnete Höhenprofile verschiedener $44 \mathrm{~nm}$ dicker Ag-Schichten, die mit der jeweils angegebenen Menge $\mathrm{ZrO}_{2}$ geglättet wurden. 
Der Verlauf der Rauigkeit mit der deponierten $\mathrm{ZrO}_{2}$-Schichtdicke (Abbildung 6.4) zeigt, dass die Rauigkeit der Oberfläche zunächst von 0,8 nm (44 nm Ag auf Si) auf einen Wert von etwa 1,0 - 1,1 nm ansteigt. Dies Verhalten kann durch die zusätzliche Bildung von amorphen $\mathrm{ZrO}_{2}$-Inseln auf der ohnehin aufgerauten Ag-Oberfläche in den Anfangsstadien des Wachstums erklärt werden. Das spricht dafür, dass die Grenzflächenenergie zwischen $\mathrm{Ag}$ und $\mathrm{ZrO}_{2}$ deutlich ungünstiger ist als die Oberflächenenergie von $\mathrm{ZrO}_{2}$, so dass das Oxid lieber auf sich selbst wächst als auf der Ag-Oberfläche.

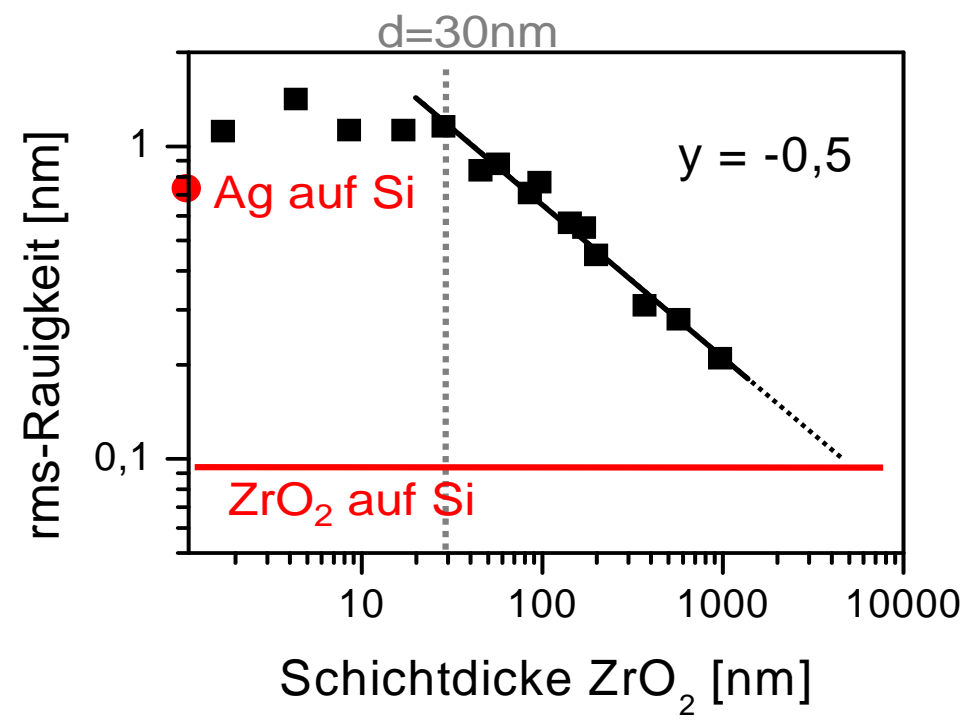

Abbildung 6.4: Rms-Rauigkeit in Abhängigkeit von der Schichtdicke des $\mathrm{ZrO}_{2}$ Films. Die Rauigkeit bleibt anfänglich konstant und nimmt ab einer Dicke von $30 \mathrm{~nm} \mathrm{ZrO}_{2} \mathrm{ab}$.

$\mathrm{Ab}$ einer Schichtdicke von etwa $30 \mathrm{~nm}$ nimmt die Rauigkeit ab. In doppeltlogarithmischer Auftragung beträgt die Steigung -0,5. In der Näherung, dass die ursprüngliche spektrale Leistungsdichte von Ag konstant war (Kapitel 3.5), deutet das auf einen Glättungsmechanismus der Ordnung $i=2$ hin (vergleiche mit Gleichung 3.18). Diese Näherung ist allerdings nur sehr begrenzt gültig, wenn man die spektrale Leistungsdichte für Ag (schwarze Kurve) in Abbildung 6.5 betrachtet, so dass die nur ein erster Hinweis ist und die Frage nach den Mechanismen später noch mal aufgegriffen werden muss. Die dickste deponierte Schicht von etwa $1 \mu \mathrm{m}$ hinterlässt eine Oberflächenrauigkeit von 0,2 nm, ist aber nicht ausreichend, um die Oberflächenstrukturen komplett zu glätten. 
Nimmt man an, dass die Rauigkeit auch für Schichtdicken größer als $1 \mu \mathrm{m}$ mit $d_{\mathrm{ZrO}_{2}}^{-0,5}$ abfällt, so ist eine Schichtdicke von etwa $4 \mu \mathrm{m}$ notwendig, um die Ag-Oberfläche eines Bildausschnittes dieser Größe komplett zu glätten, d.h. die Rauigkeit von $\mathrm{ZrO}_{2}$ auf glattem Si zu erreichen. Es muss also eine erstaunlich große Schichtdicke deponiert werden, um die ursprüngliche Rauigkeit von lediglich 0,8 nm auszuglätten. Daraus lässt sich folgern, dass $\mathrm{ZrO}_{2}$ möglicherweise nicht das geeignetste Material zur Glättung von Ag ist, allerdings hat es hier den großen Vorteil, dass sich die einzelnen Zwischenschritte hervorragend im Detail beobachten lassen.

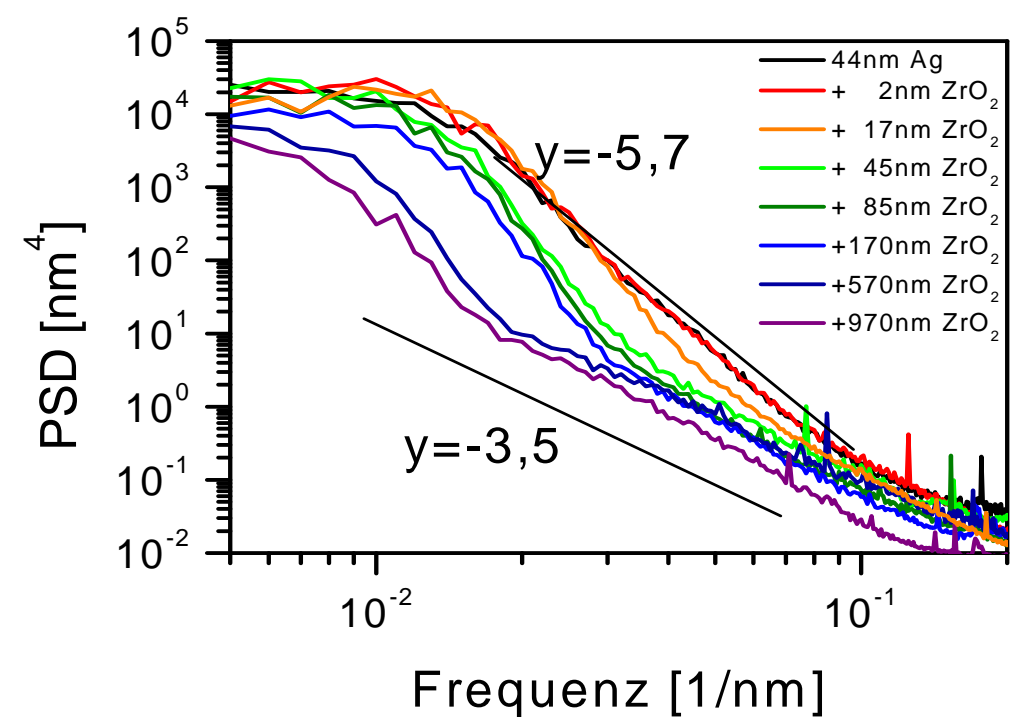

Abbildung 6.5: Veränderung der spektralen Leistungsdichte der Ag-Schicht (schwarz) im Verlauf der Glättung mit $\mathrm{ZrO}_{2}$ unterschiedlicher Schichtdicke.

Um die Rauigkeitsveränderungen in Abhängigkeit von der Morphologie auf der Oberfläche besser zu verstehen, sollen als Nächstes die Oberflächen der Schichten frequenzaufgelöst im Fourierraum betrachtet werden (Abbildung 6.5). Ausgehend von der spektralen Leistungsdichte der reinen Ag-Schicht beobachtet man mit zunehmender $\mathrm{ZrO}_{2}$ Schichtdicke eine Abnahme der spektralen Leistungsdichte. Dies ist verständlich, da die Rauigkeit mit der Fläche unter den Kurven verknüpft ist (Gleichung 3.4) und somit die beobachtete Glättung ein Absinken der PSDs bewirkt. Die erwartete Endkurve entspricht dem Leistungsspektrum einer sehr dicken reinen $\mathrm{ZrO}_{2}$-Schicht auf glattem Substrat (Abbildung 5.26), wird hier aber auch bei der dicksten deponierten Schicht nicht erreicht. Bereits bei der Glättung der Wellenstrukturen (Kapitel 5.5) wurde eine ausgeprägte 
Frequenzabhängigkeit beobachtet, da die Inseln sich mit zunehmender Schichtdicke verbreitern. Dies bestätigt die Fourieranalyse: Bei dünnen Schichtdicken (z.B. 2 nm (rot) und $17 \mathrm{~nm}$ (orange) in Abbildung 6.5) ist gut zu erkennen, dass die Schichtdeposition zunächst eine Glättung der hohen Frequenzen, also der kleinen Strukturen auf der Oberfläche bewirkt, da die Spektren dort zuerst absinken. Die tiefen Frequenzen dagegen bleiben erhalten bzw. werden noch etwas verstärkt, was wieder auf ein anfängliches Inselwachstum der $\mathrm{ZrO}_{2}$-Schicht schließen lässt und den beobachteten Anstieg der Rauigkeit erklärt. Die stark frequenzabhängige Glättung führt wie in der Theorie vorhergesagt (vergleiche Kapitel 3.4) zu einem zweiten Knick in den Spektren im mittelbis hochfrequenten Bereich, der sich mit steigender Schichtdicke zu tieferen Frequenzen verschiebt. Oberhalb des Knicks sind die von der ursprünglichen Ag-Oberfläche stammenden Frequenzen bereits vollständig ausgeglättet, so dass dieser Teil der Kurve den $\mathrm{ZrO}_{2}$-Spektren auf glattem Si entspricht. Die Abknickfrequenz entspricht der kleinsten gerade noch vollständig ausgeglätteten Frequenz $k_{2}$, d.h. der größten vollständig ausgeglätteten Wellenlänge $\lambda_{2}$ auf der Oberfläche. Sie ist in Abbildung 6.6 (2. Knick) aufgetragen gegen die Schichtdicke von $\mathrm{ZrO}_{2}$. Der lineare Fit zeigt, dass folgender Zusammenhang gilt: $\lambda_{2}(d) \sim d^{0,26}$.

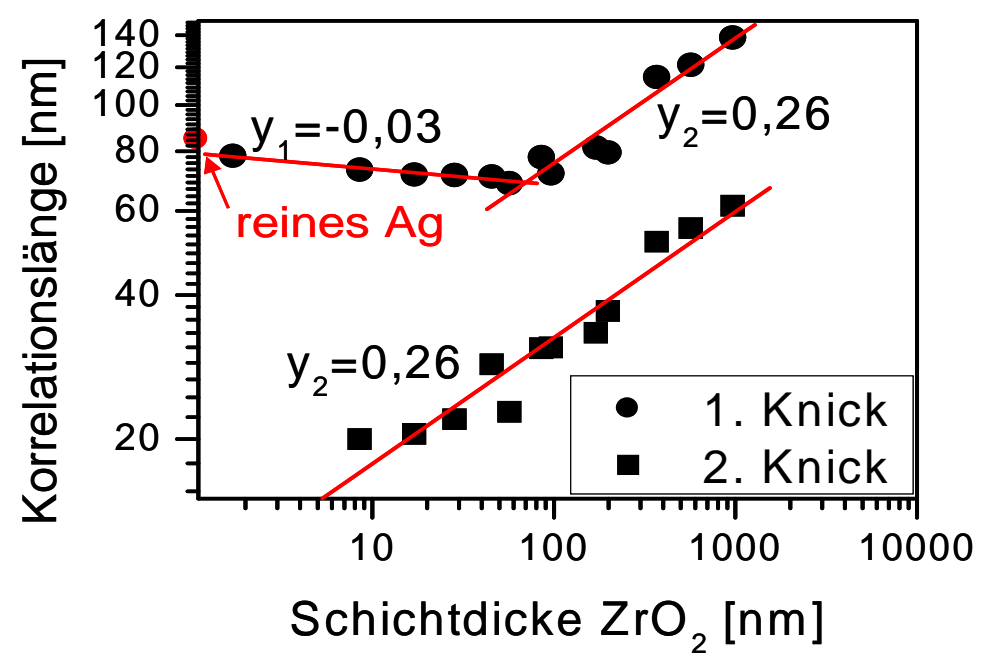

Abbildung 6.6: Verlauf der lateralen Korrelationslängen, wie sie aus den spektralen Leistungsdichten bestimmt wurden. Das erste Abknicken kann hierbei mit der charakteristischen lateralen Strukturgröße auf der Oberfläche identifiziert werden, der zweite Knick mit der größten gerade noch ausgeglätteten Wellenlänge. 
Das erste Abknicken der Spektren $\left(k_{c}\right)$ bei tiefen Frequenzen beschreibt auch hier die dominierende Strukturgröße $\lambda_{c}$, eine wichtige Korrelationslänge auf der Oberfläche. Für die reine Ag-Oberfläche liegt sie bei $(86 \pm 5) \mathrm{nm}$. Abbildung 6.6 zeigt, dass sie anfänglich in etwa konstant bleibt, also die mittlere Inselgröße von der Schichtdeposition und den Glättungsmechanismen kaum verändert wird. Ab einer deponierten $\mathrm{ZrO}_{2}$-Dicke von etwa $60 \mathrm{~nm}$ beginnt die Glättung dieser Hauptstruktur, so dass sich die dominierende Strukturgröße der Probe zu kleineren Frequenzen hin verschiebt. Die Korrelationslänge steigt ab diese Schichtdicke mit demselben Skalengesetz an wie die Kurve des zweiten Knicks und beschreibt ab dem Zeitpunkt des Anstieges die größte noch gar nicht geglättete Wellenlänge $\lambda_{1}$. Der Versatz zwischen den Kurven ist also der Wellenlängenbereich ( $\lambda_{2}$ bis $\lambda_{1}$ ), in dem die Glättung stattfindet (vergleiche Kapitel 3.4, Abbildung 3.4). Er hat hier eine Breite von etwa $40 \mathrm{~nm}$.
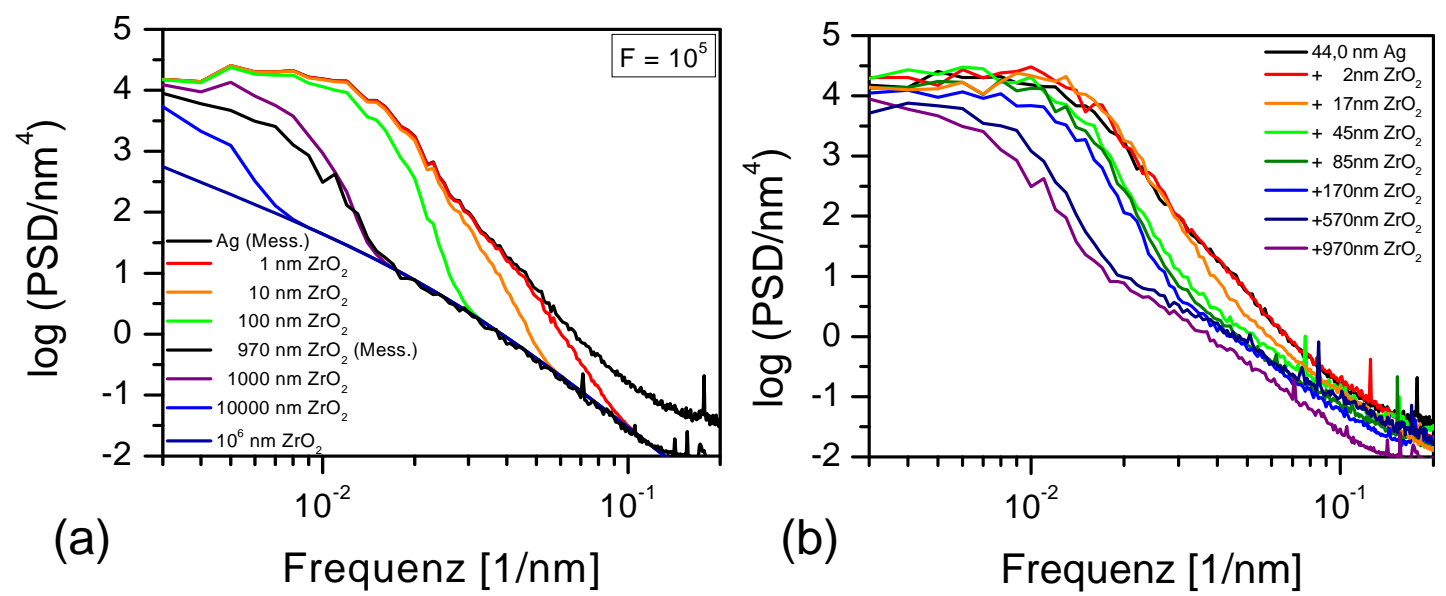

\begin{abstract}
Abbildung 6.7: (a) Simulation der Glättung mithilfe der Gleichung 3.14. Als Ausgangskurve wurde die spektrale Leistungsdichte der gemessenen Ag-Schicht genommen. Die freien Parameter wurden mithilfe des Spektrums der mit $970 \mathrm{~nm}$ $\mathrm{ZrO}_{2}$ geglätteten Probe angepasst. (b) zeigt zum Vergleich noch mal die gemessenen spektralen Leistungsdichten.
\end{abstract}

Die verschiedenen Stadien der Glättung einer rauen Oberfläche werden im linearen Modell durch Gleichung 3.14 beschrieben. Setzt man als Anfangsspektrum die gemessene spektrale Leistungsdichte einer reinen Ag-Schicht ein, so lassen sich die einzelnen Stadien der Glättung, wie im Theorieteil (Kapitel 3.4) und bereits bei den Wellenstrukturen (Kapitel 5.5) beschrieben, simulieren. Die freien Parameter $R, b_{2}$ und $b_{4}$ wurden mithilfe 
der mit $970 \mathrm{~nm} \mathrm{ZrO}_{2}$ geglätteten Schicht angepasst, indem der hintere, bereits vollständig geglättete Teil der Kurve mit Gleichung 3.13 gefittet wurde. Dieser Fit ergab, dass bis zu einer Grenzwellenlänge von etwa $40 \mathrm{~nm}$ hauptsächlich Diffusion (Ordnung 4, [Herr50]) für die Strukturbildung verantwortlich ist, darüber dominieren Sputtererosion oder ballistisches Glätten (Ordnung 2, [Brad88], [Mose05]). In Abbildung 6.7(a) sind die Ergebnisse der Simulation dargestellt zusammen mit den gemessenen Spektren der Anfangskurve und der mit $970 \mathrm{~nm}$ geglätteten Oberfläche (beide in schwarz). Anhand des Vergleichs der theoretischen und der experimentell bestimmten Abknickpunkte konnte die reziproke Depositionsrate $F^{-1}=10^{-5}$ bestimmt und den Kurven damit eine Schichtdicke $d$ zugeordnet werden. Vergleicht man den theoretischen Kurvenverlauf mit dem gemessenen (Abbildung 6.7(b)), so wird der prinzipielle Verlauf der Spektren mit den erwarteten Abknickpunkten und dem schichtdickenabhängigen Übergangsbereich, in dem die Glättung stattfindet, gut wiedergegeben. Wie bereits in Kapitel 5.5 dargestellt, kann aber auch hier das zusätzliche gleichmäßige Absinken der Spektren mit steigender Schichtdicke nicht simuliert werden. Eine Ursache könnte eine Schichtdickenabhängigkeit der $b_{\mathrm{i}}$-Werte sein. Möglicherweise führt aber auch die lineare Näherung im Modell zu diesen Unterschieden.

Zusammenfassend konnte gezeigt werden, dass die Deposition von $\mathrm{ZrO}_{2}$ eine AgOberfläche der Anfangsrauigkeit 0,8 nm glätten kann, wobei die Glättung selber frequenzabhängig ist und von der deponierten Schichtdicke abhängt: Kleine Strukturen auf der Oberfläche werden bereits bei kleinen deponierten $\mathrm{ZrO}_{2}$-Dicken ausgeglättet, während langwellige Unebenheiten sehr große Schichtdicken, in diesem Fall von bis zu $4 \mu \mathrm{m}$ erfordern können. Vergleicht man das Wachstum von $\mathrm{ZrO}_{2}$ auf glattem Si bzw. Si-Wellen mit dem auf Ag, so scheint es entscheidende Unterschiede in den Grenzflächenenergien zu geben, die bewirken, dass $\mathrm{Si}$ sofort benetzt und geglättet wird, während $\mathrm{ZrO}_{2}$ auf $\mathrm{Ag}$ erst Inseln bildet und leicht aufraut, bevor ein Glättungsprozess einsetzen kann. Sobald geschlossene $\mathrm{ZrO}_{2}$-Schichten vorliegen, wächst das Oxid wahrscheinlich lagenartig auf sich selbst, was bewirkt, dass die Schichten auf glattem Si nicht weiter aufrauen und die bereits rauen Oberflächen (Si-Wellen / Ag-Schicht) zunächst bestehen bleiben und dann langsam ausgeglättet werden. Die Simulation der spektralen Leistungsdichten zeigt, dass sich der Glättungsprozess mithilfe von Gleichung 3.14 prinzipiell gut beschreiben lässt, 
allerdings gibt es ein frequenzunabhängiges Absinken der Kurven, das von der Theorie nicht vorhergesagt wird.

Im folgenden Unterkapitel soll die Glättung abhängig von der Morphologie der ursprünglichen Ag-Oberfläche betrachtet werden. Dafür werden die unterschiedlich dicken Ag-Schichten auf Si als Substrat verwendet, die bereits in Kapitel 4.2 beschrieben wurden. Sie unterscheiden sich einerseits in der Anfangsrauigkeit, aber auch in der dominierenden Korn- bzw. Strukturgröße, die von den unterschiedlichen Wachstumsstadien des Ag beeinflusst werden.

\subsection{Abhängigkeit von der Oberflächenbeschaffenheit des Substrats}

In Kapitel 6.1 wurde das Glättungsverhalten einer vorgegebenen rauen Oberfläche in Abhängigkeit von der deponierten Materialmenge untersucht. So konnten die einzelnen Stadien bis zur vollständigen Glättung beobachtet und hinsichtlich ihrer Morphologie und des theoretischen Verhaltens frequenzaufgelöst analysiert werden. Im Folgenden soll nun die vorherrschende Strukturgröße der rauen Unterlage möglichst systematisch verändert und ihr Einfluss auf eine erfolgreiche Glättung analysiert werden. Kapitel 4.2 hat gezeigt, dass die Variation der Strukturgröße einfach möglich ist, indem unterschiedliche Mengen Ag auf Si deponiert werden. Einziger Nachteil hierbei ist, dass die dünnen Ag-Schichten noch nicht geschlossen sind und das unterschiedliche Wachstumsverhalten von $\mathrm{ZrO}_{2}$ auf Ag und Si bei der Interpretation berücksichtigt werden muss. Die Auswirkungen der Strukturgröße auf das Glättungsverhalten wird untersucht, indem stets dieselbe Schichtdicke $\mathrm{ZrO}_{2}$ auf die unterschiedlichen Oberflächen aufgebracht wird. Hierfür wurde eine mittlere Dicke von $44 \mathrm{~nm}$ gewählt, da im vorherigen Unterkapitel bei dieser Dicke bereits eine deutliche Glättung für Strukturen bis etwa $30 \mathrm{~nm}$ Größe beobachtet werden konnte (vergleiche Abbildung 6.3). Die dominierenden Korngrößen von Ag-Schichten unterschiedlicher Dicke variieren zwischen 20 nm und 90 nm (für Dicken zwischen 0,1 nm 
und $100 \mathrm{~nm}$, vergleiche Abbildung 4.6), sodass hier deutlich unterschiedliche Resultate aufgrund des beobachteten frequenzabhängigen Glättungsverhaltens zu erwarten sind.

Abbildung 6.8 (a) - (f) zeigt jeweils die Probenoberfläche vor (oben) und nach (unten) der Glättung mit $44 \mathrm{~nm} \mathrm{ZrO}_{2}$. Die jeweiligen Ag-Schichtdicken sind angegeben. Es ist gut zu erkennen, dass die mittlere Inselgröße der Ag-Schichten mit zunehmender Schichtdicke ansteigt (vergleiche Kapitel 4.2).

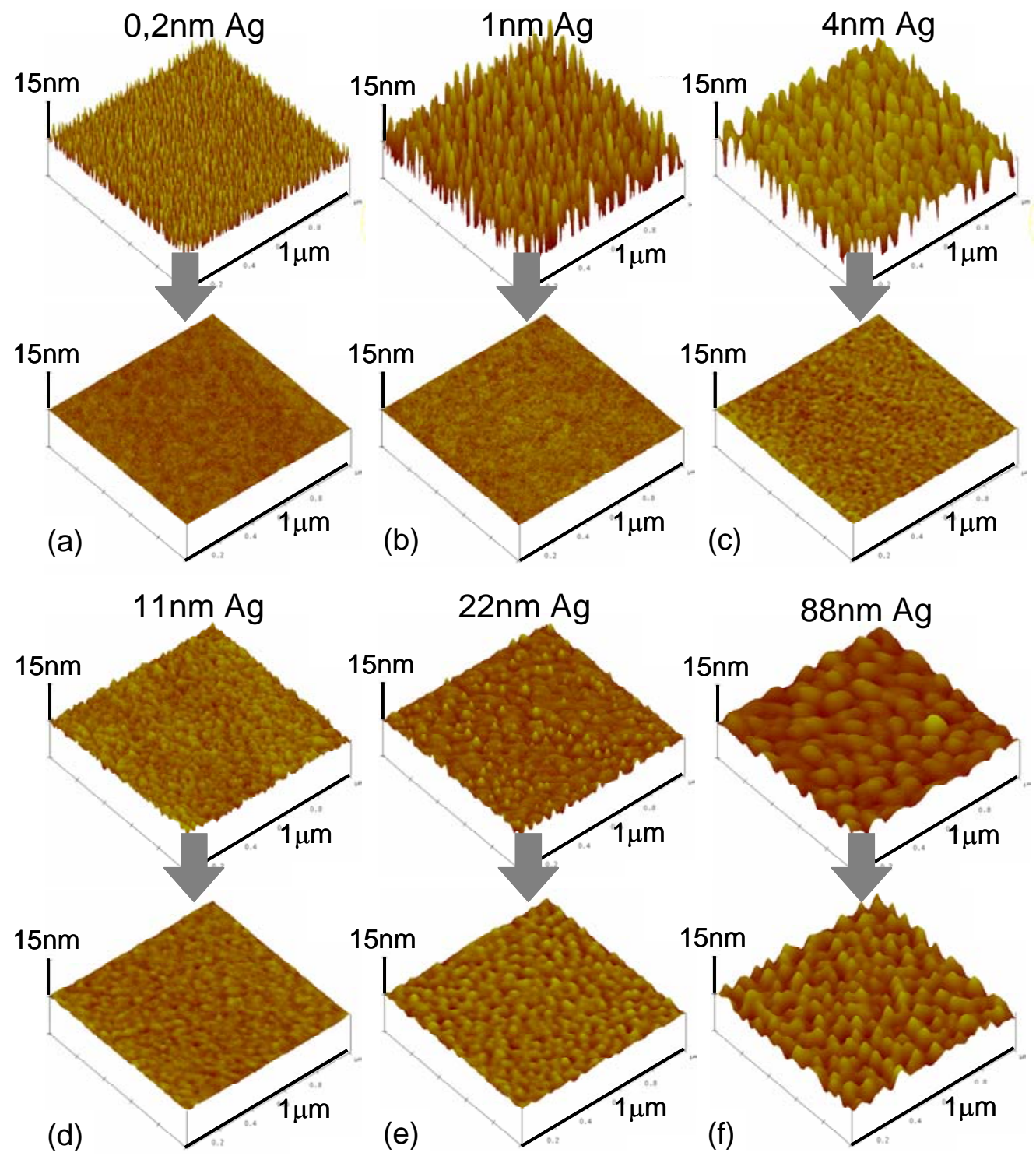

Abbildung 6.8: AFM-Aufnahmen verschiedener Oberflächen vor (jeweils oben) und nach der Glättung mit $44 \mathrm{~nm} \mathrm{ZrO}_{2}$. Von (a) bis (f) steigt mit der deponierten Ag-Schichtdicke (jeweils angegeben) die dominante Strukturgröße auf der Oberfläche. Die Glättung selbst ist anfangs komplett möglich, später werden nur noch die kleineren Oberflächenwellenlängen ausgeglättet. 
Es zeigt sich, dass die Deposition von $\mathrm{ZrO}_{2}$ in den Fällen (a) bis (c) sehr glatte Oberflächen hinterlässt. Je dicker die Ag-Schicht des Substrats wird, desto unvollständiger ist dagegen die Glättung. Um die Ursache hierfür zu finden, ist in Abbildung 6.9 der Verlauf der rms-Rauigkeit gegen die Schichtdicke aufgetragen. Die rms-Rauigkeit der Oberfläche vor der Glättung (schwarze Kurve, vergleiche Kapitel 4.2) unterliegt starken Schwankungen, die Rauigkeit nach der Glättung (rot) zeigt dagegen einen kontinuierlichen Verlauf. Sie steigt umso mehr an, je dicker der ursprüngliche Ag-Film war. Der Graph zeigt demnach sehr anschaulich, dass der entscheidende Faktor für eine erfolgreiche Glättung nicht die Anfangsrauigkeit der Oberfläche sein kann, da Rauigkeiten von etwa $4 \mathrm{~nm}$ bei geringen Ag-Schichtdicken problemlos ausgeglättet werden, während wesentlich geringere Rauigkeiten bei dicken Schichten nur wenig oder fast gar nicht geglättet werden.

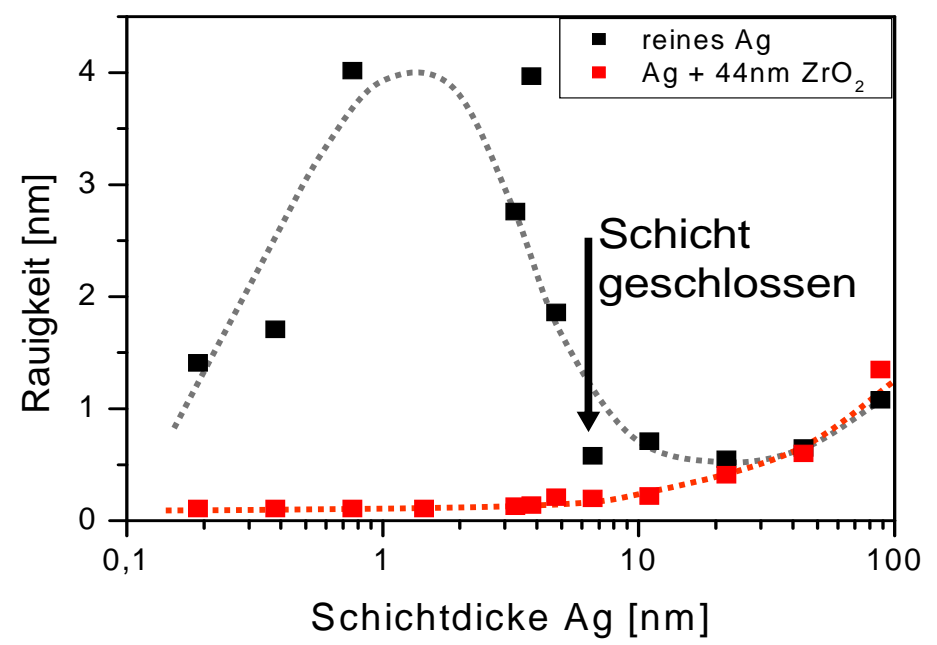

Abbildung 6.9: Der Verlauf der rms-Rauigkeit mit der Ag-Schichtdicke vor (schwarz) und nach (rot) der Glättung mit $44 \mathrm{~nm} \mathrm{ZrO}_{2}$. Als Bildausschnitt wurde $1 \times 1 \mu \mathrm{m}^{2}$ gewählt.

Die Ursache für das unterschiedliche Glättungsverhalten ist folglich bei einem anderen Parameter zu suchen, der sich kontinuierlich mit der ursprünglich deponierten AgSchichtdicke verändern muss. Dafür kommt die mittlere Strukturgröße, bzw. die anfängliche Frequenzverteilung, auf der Oberfläche in Frage, es können aber auch die Substratmaterialien (Ag, bzw. Si) eine Rolle spielen. Dies soll im Folgenden anhand der spektralen Leistungsdichten und der Korrelationslängen analysiert werden und die Effizienz der Glättung in Zusammenhang gebracht werden mit den Eigenschaften der jeweiligen Proben. In Abbildung 6.10(a) sind die Spektren einiger (jeweils mit $44 \mathrm{~nm}$ 
$\mathrm{ZrO}_{2}$ ) geglätteter Proben dargestellt, jeweils angegeben ist die Schichtdicke der ursprünglichen Ag-Schicht. Während die Glättung bei dünnen Ag-Schichten bis 1,5 nm komplett ist (kein zweiter Knick im Spektrum), werden bei Proben ab $3 \mathrm{~nm}$ Ag-Dicke kleine Frequenzen, d.h. große Strukturen auf der Probe nur noch unvollständig oder gar nicht mehr ausgeglättet, der dafür charakteristische zweite Knick tritt bei Frequenzen von etwa $0,03 \mathrm{~nm}^{-1}$ bis $0,04 \mathrm{~nm}^{-1}$ auf. Im Folgenden sollen beide Fälle zunächst getrennt betrachtet werden.
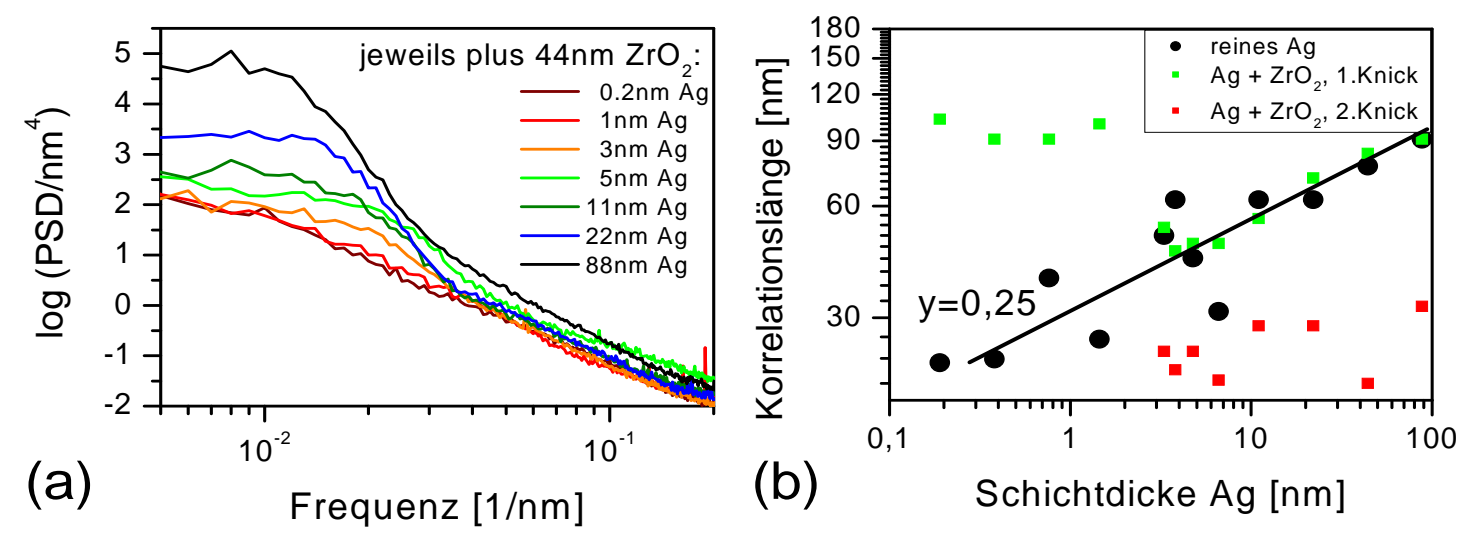

\begin{abstract}
Abbildung 6.10: (a) Die spektralen Leistungsdichten der geglätteten Schichten verändern sich kontinuierlich mit der ursprünglich aufgebrachten Ag-Menge: Je mehr Ag deponiert wurde, desto unvollständiger ist die Glättung nach Deposition von $44 \mathrm{~nm} \mathrm{ZrO}_{2}$. (b) Die Korrelationslängen der ursprünglichen Oberflächen (schwarz), sowie der mit $44 \mathrm{~nm}$ geglätteten Oberflächen (grün und rot) sind aufgetragen gegen die Dicke der Ag-Schicht. Der zweite Knick tritt erst bei Schichtdicken von 3 nm oder mehr auf.
\end{abstract}

Im ersten Fall soll die vollständige Glättung der sehr dünnen Ag-Schichten (bis $2 \mathrm{~nm}$ Dicke) betrachtet werden. Diese Ag-Filme bestehen noch aus sehr kleinen, getrennten Inseln. Zum Vergleich ist in Abbildung 6.11(a) beispielhaft die spektrale Leistungsdichte der $1 \mathrm{~nm}$ dicken Schicht vor (schwarz) und nach der Glättung (rot) abgebildet. Die rote Kurve entspricht der Leistungsdichte einer $\mathrm{ZrO}_{2}$-Schicht auf glattem Substrat, was die vollständige Glättung belegt. Vermisst man die Abknickpunkte in den Spektren, so erhält man den Verlauf der ersten und der zweiten Korrelationslänge Abbildung 6.10(b), die die dominante Strukturgröße auf der Oberfläche (schwarz für die Ag-Unterlage (siehe auch Kapitel 4.2), grün für die geglätteten Schichten) und die kleinste 
vollständig geglättete Frequenz beschreiben (rot). Für die dünnen Schichten (bis etwa 2 nm Ag-Schichtdicke) zeigt der Verlauf der grünen Kurve, dass die dominante Strukturgröße der $\mathrm{ZrO}_{2}$-Schicht konstant bei etwa $95 \mathrm{~nm}$ liegt. Denselben Hügeldurchmesser zeigen $\mathrm{ZrO}_{2}$-Schichten, die auf glattem Si deponiert wurden. Ein zweiter Knick tritt noch nicht auf. Diese Ergebnisse erwecken den Eindruck, dass der $\mathrm{ZrO}_{2}$-Film hier unabhängig von den Ag-Inseln so wächst, wie es auch auf Si beobachtet wurde.

Sobald die Ag-Schichten koaleszieren und perkolieren, d.h. die dominante Strukturgröße und der von Ag bedeckte Flächenanteil deutlich zunehmen (dies ist ab etwa 3 nm Ag-Schichtdicke der Fall), ist die Glättung nicht mehr erfolgreich. Dies ist der zweite Fall, der betrachtet werden soll. Hier tritt in den spektralen Leistungsdichten der übliche zweite Knick bei der Frequenz $k_{2}$ auf (vergleiche Abbildung 6.10(a)), der mit jedem noch unvollständigen Glättungsprozess einhergeht. Der Übergang zwischen diesen beiden Bereichen ist fließend.
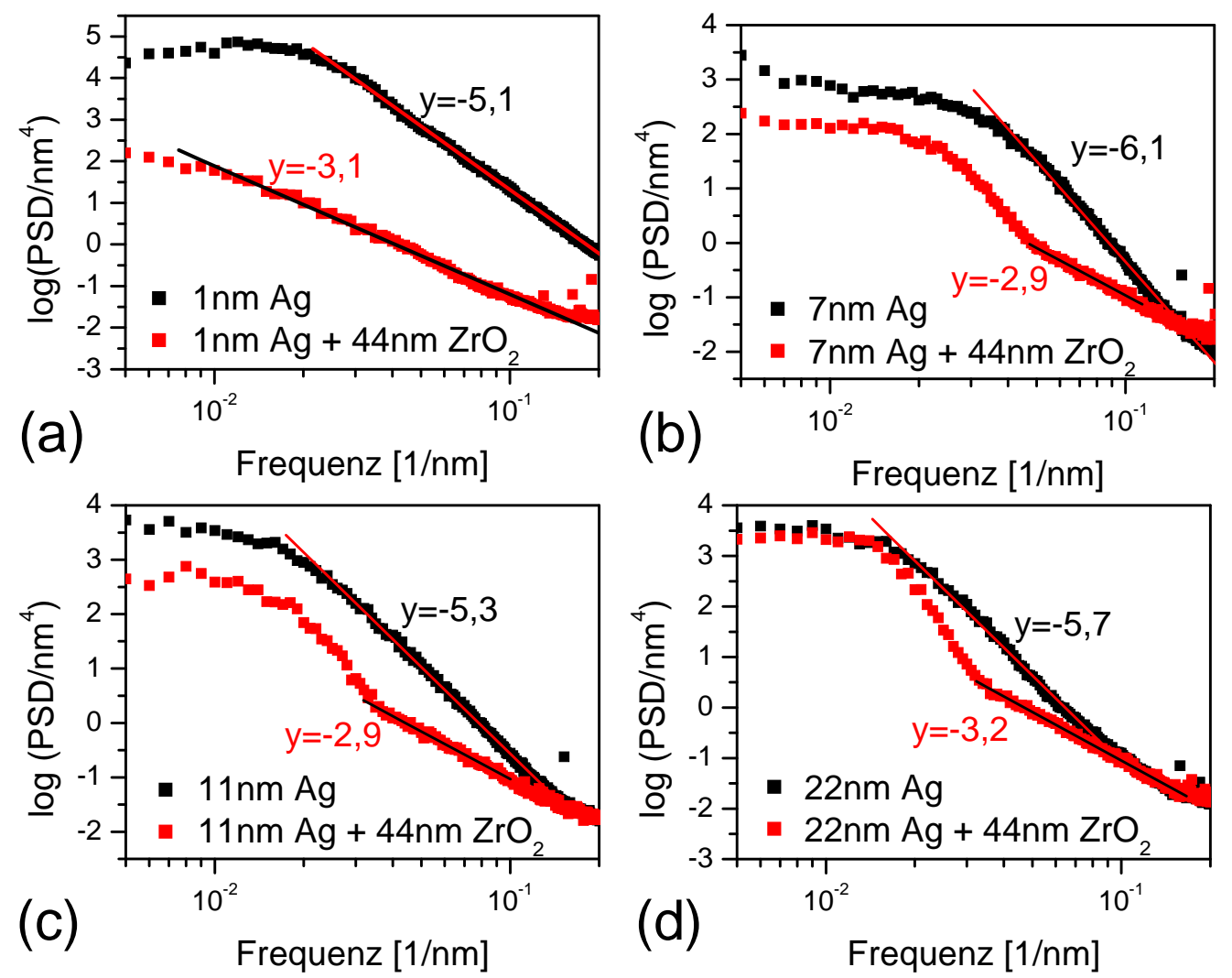

Abbildung 6.11: In schwarz ist jeweils die spektrale Leistungsdichte der AgSchicht angegebener Dicke dargestellt, in rot die Leistungsdichte der mit $44 \mathrm{~nm}$ $\mathrm{ZrO}_{2}$ geglätteten Oberfläche. 
Zum besseren Verständnis der Vorgänge auf dem Substrat soll auch hier für einige Schichtdicken die spektrale Leistungsdichte vor und nach der Glättung verglichen werden (Abbildung 6.11, Spektren (b) - (d)): Die Spektren (b) - (d) zeigen, dass die Glättung mit steigender Ag-Schichtdicke immer weniger erfolgreich ist, da das ursprüngliche Spektrum immer weniger abgesenkt wird. Dies liegt daran, dass die dominante Strukturgröße (erste Korrelationslänge) der jeweiligen Ag-Spektren (schwarze Kurven) bei immer tieferen Frequenzen liegt und somit mit der deponierten $\mathrm{ZrO}_{2}$-Schichtdicke von $44 \mathrm{~nm}$ immer weniger geglättet werden kann. Dies passt zu den bisherigen Ergebnissen, dass stets erst hohe Frequenzen (kleine Strukturen) geglättet werden: Je größer eine Struktur auf der Oberfläche ist, umso weniger kann sie mit einer vorgegebenen Menge $\mathrm{ZrO}_{2}$ geglättet werden, d.h. umso rauer bleibt die Oberfläche. Da die Glättung nicht erfolgreich ist, ist dementsprechend die dominante Strukturgröße der mit $\mathrm{ZrO}_{2}$ beschichteten Proben (vergleiche Abbildung 6.10(b), grüne Kurve) ähnlich groß wie die der ursprünglichen AgOberfläche (schwarze Kurve). Die größte vollständig geglättete Wellenlänge (zweite Korrelationslänge, rote Kurve) liegt für die unvollständig geglätteten Proben stets zwischen $20 \mathrm{~nm}$ und $30 \mathrm{~nm}$. Das war zu erwarten, da auf die rauen Oberflächen konstant $44 \mathrm{~nm} \mathrm{ZrO}_{2}$ deponiert wurden und diese Größe allein mit der zur Glättung verwendeten $\mathrm{ZrO}_{2}$-Schichtdicke variiert. Die Schwankungen erklären sich über leichte Unterschiede der Depositionsparameter.

Allerdings ist nicht nur die mittlere Strukturgröße der Ag-Oberfläche für das unterschiedliche Glättungsverhalten verantwortlich: Vergleicht man die Spektren in Abbildung 6.11, ist es auffällig, dass die spektrale Leistungsdichte von Ag in (a) deutlich höher liegt als in den Graphen (b) - (d) und auch die dominante Inselgröße etwas größer ist, so dass es prinzipiell auch in (a) nicht möglich sein sollte, die Strukturen mit $44 \mathrm{~nm}$ $\mathrm{ZrO}_{2}$ komplett auszuglätten. Dass die Glättung hier trotzdem so gut funktioniert, muss daher daran liegen, dass die Ag-Schichten noch nicht geschlossen sind. Die Ergebnisse lassen sich folglich unter der Annahme verstehen, dass $\mathrm{ZrO}_{2}$ gleichmäßig auf der glatten Si-Oberfläche wächst und dabei die vereinzelten Ag-Inseln weitgehend „ignoriert“, da die Ausbildung einer $\mathrm{ZrO}_{2}$-Ag-Grenzfläche energetisch ungünstig ist. Das auf den Ag-Inseln deponierte Material muss dabei großteils in die Täler diffundieren, so dass die Inseln überwachsen werden können ohne einen Beitrag zur Endrauigkeit der Oberfläche zu leisten. Dies deckt sich mit den Überlegungen zu den vollständig geglätteten Proben (Ag- 
Schichtdicken bis $3 \mathrm{~nm}$, siehe oben). Je dicker die Ag-Schichten werden, desto weniger freie Si-Oberfläche gibt es, was dazu führt, dass das vollständige Überwachsen durch die endliche Diffusionslänge von $\mathrm{ZrO}_{2}$ auf $\mathrm{Ag}$ verhindert wird. Stattdessen bilden sich zunächst $\mathrm{ZrO}_{2}$-Inseln auf Ag, später geschlossene Schichten, wobei analog wie in Kapitel 6.1 die rauen Strukturen der Oberfläche zunächst bestehen bleiben und erst nach und nach mit zunehmender $\mathrm{ZrO}_{2}$-Schichtdicke ausgeglättet werden. Ein endgültiger Beweis für diese Theorie war im Rahmen dieser Arbeit nicht möglich. Allerdings wäre es ein interessanter Ansatz für eine neue Diplom- oder Doktorarbeit, systematisch die Glättung von Strukturen zu untersuchen, die aus zwei unterschiedlichen Materialien aufgebaut sind.

Zusammenfassend hat Kapitel 6 gezeigt, dass für eine erfolgreiche Glättung folgende Parameter aufeinander abgestimmt werden müssen: Für gegebene Materialien bestimmt die Frequenzverteilung auf der rauen Oberfläche die zur Glättung benötigte Schichtdicke. Je kleiner die Strukturen sind, desto schneller, d.h. mit umso geringeren Schichtdicken können diese ausgeglättet werden. Des Weiteren kann eine geeignete Wahl der Materialien die Effizienz der Glättung entscheidend beeinflussen. So ist es aufgrund der Grenzflächenenergien einfacher eine Si-Oberfläche mit $\mathrm{ZrO}_{2}$ zu glätten als eine AgOberfläche. 


\section{Zusammenfassung}

Ziel dieser Arbeit war, die Glättung rauer Oberflächen durch Deposition dünner Schichten zu untersuchen. Wie im zweiten Kapitel der Arbeit beschrieben wurde, kann aus der Theorie zur wachstumsbedingten Glättung und Aufrauung geschlossen werden, dass für die erfolgreiche Glättung einer rauen Oberfläche geeignete Materialien hauptsächlich zwei Kriterien erfüllen müssen: Einerseits muss die intrinsische Rauigkeit (Eigenrauigkeit der Materialien auf glatten Substraten) sehr gering sein, da dies die Rauigkeit ist, die nach dem vollständigen Glättungsprozess zurück bleibt. Andererseits sollen die auf der Unterlage vorhandenen Rauigkeiten hinreichend schnell ausgeglättet werden, damit die benötigte Schichtdicke möglichst gering ist. Ob dies der Fall ist, wird bestimmt durch die auf der Oberfläche auftretenden atomaren Transportmechanismen der Ordnung $i$. Der Glättungsverlauf kann daher für verschiedene Materialklassen sehr unterschiedlich sein und die Wahl des Glättungsmaterials muss auf das verwendete Substrat abgestimmt werden. Die Theorie sagt des Weiteren voraus, dass der Glättungsprozess frequenzabhängig ist. Hierbei gibt die Charakteristik der Frequenzabhängigkeit Hinweise auf die Mechanismen, die für die Glättung verantwortlich sind.

In der Tat wurden im Rahmen dieser Arbeit mehrere Materialien gefunden, mit denen die vollständige Glättung einer rauen Oberfläche möglich ist. Dabei konnten minimale Rauigkeiten von 0,2 nm erzeugt und die Vorhersagen der Theorie überprüft werden. Auch die frequenzabhängige Glättung wurde systematisch und im Detail beobachtet, die Glättungsmechanismen bestimmt, sowie der Glättungsvorgang simuliert und mit den Experimenten verglichen.

Zur Untersuchung der Materialabhängigkeit der Glättung im Real- und Fourierraum wurden hierbei Si-Wellenstrukturen mit einer Periodizität von $(55 \pm 5) \mathrm{nm}$, einer mittleren 
Höhe von $4 \mathrm{~nm}$ und einer Rauigkeit von etwa 1,3 $\mathrm{nm}$ als raue Unterlage verwendet. Diese Art des Substrats ermöglichte es, die Glättung bis zu einer bestimmten, sehr markanten Oberflächenfrequenz zu untersuchen und dafür notwendige Schichtdicke zu bestimmen. Diese ist zusammen mit der erzeugten Endrauigkeit ein wichtiger Parameter für die Effizienz der Glättung. Als Glättungsmaterialien wurden ein kristallines Metall (Ag), amorpher C, zwei Polymere (PMMA und PC) und ein amorphes Oxid $\left(\mathrm{ZrO}_{2}\right)$ studiert.

Bei den Untersuchungen hat sich gezeigt, dass sowohl Ag als auch C jeweils eines der beiden oben genannten Kriterien nicht ausreichend erfüllen, um als Glättungsmaterialien für raue Si-Oberflächen der gewählten Strukturgrößen in Frage zu kommen. Ag besitzt eine sehr hohe intrinsische Rauigkeit auf glattem $\mathrm{Si}$, die durch das kristalline Inselwachstum und eine sehr schnelle Diffusion zustande kommt. Die minimal mögliche Rauigkeit beträgt $0,8 \mathrm{~nm}$ und tritt bei glatten Substraten dann auf, wenn die Filme gerade geschlossen sind. Im Vergleich zu den anderen untersuchten Materialien, ist dieser Wert sehr hoch. Deponiert man Ag auf den Wellenstrukturen, so werden die Wellen zwar schon nach der Deposition von nur $8 \mathrm{~nm}$ Ag überlagert, allerdings sind die neu erzeugten Rauigkeiten von etwa 1,4 nm nicht geringer als die ursprüngliche Rauigkeit der Si-Wellen. Fourieranalysen belegen, dass lediglich die Art der Oberflächenstrukturen von Wellen zu statistischen Strukturen umgewandelt wird. Somit eignet sich Ag aufgrund seiner hohen Eigenrauigkeit nicht zum Glätten dieser kleinen Oberflächenstrukturen.

Im Fall von C liegt die intrinsische Rauigkeit auf glatten Substraten über einen weiten Schichtdickenbereich hinweg konstant bei $0,2 \mathrm{~nm}$ mit charakteristischen Strukturgrößen zwischen $20 \mathrm{~nm}$ und $40 \mathrm{~nm}$. Über ein Anfitten der spektralen Leistungsdichten konnten gezeigt werden, dass bis zu einer Strukturgröße von etwa $43 \mathrm{~nm}$ Diffusion (Ordnung 4) der dominante Glättungsmechanismus ist, danach tragen hauptsächlich Sputtererosion und ballistisches Glätten (Ordnung 2) zur Strukturbildung bei. Bei den Wellenstrukturen beobachtet man zwar eine kontinuierliche Abnahme der Wellenhöhe, allerdings waren auch die dicksten Schichten (230 nm) nicht ausreichend, um die Wellen vollständig zu glätten. Die rms-Rauigkeit zeigt einen sehr langsamen exponentiellen Abfall. Da die mit PLD deponierten C-Schichten stark verspannt sind und schlecht auf Si haften, konnten keine Schichtdicken größer als $230 \mathrm{~nm}$ aufgebracht werden. Somit ist auch C trotz der geringen Eigenrauigkeit für die Glättung der vorgegebenen SiStrukturen nicht brauchbar. Möglicherweise ist C doch als Glättungsmaterial einsetzbar, 
wenn andere Substratmaterialien und ggf. auch eine andere Herstellungsmethode gewählt werden. Dies war allerdings nicht Teil der Arbeit.

Im Gegensatz zu Ag und $\mathrm{C}$ ist es bei drei Systemen (PC, PMMA und $\mathrm{ZrO}_{2}$ ) tatsächlich gelungen, die vorgegebenen Rauigkeiten des Substrats effizient zu glätten und die Oberflächenprozesse systematisch zu untersuchen: PC-Schichten auf glattem Si rauen mit steigender Schichtdicke leicht auf, dickere Filme haben trotzdem eine geringe intrinsische Rauigkeit von $0,3 \mathrm{~nm}$ bis $0,4 \mathrm{~nm}$ mit Strukturgrößen zwischen $20 \mathrm{~nm}$ und $80 \mathrm{~nm}$. Die Wellenstrukturen werden ähnlich wie bei C sukzessive geglättet und sind bereits nach der Deposition von etwa $70 \mathrm{~nm}$ nicht mehr erkennbar. Bei der Entwicklung der Rauigkeit mit der Schichtdicke äußert sich dies in einem schnellen exponentiellen Abfall bis bei $70 \mathrm{~nm}$ Schichtdicke eine Rauigkeit von $0,4 \mathrm{~nm}$ erreicht ist, die der Eigenrauigkeit von PC entspricht. Die aus der Theorie erwartete Frequenzabhängigkeit der Glättung konnte im Experiment nachgewiesen werden und äußert sich bei den Wellenstrukturen darin, dass in der spektralen Leistungsdichte die Maxima höherer Ordnung bereits bei geringeren Schichtdicken verschwinden als das Hauptmaximum.

Auch die Glättung mit $\mathrm{ZrO}_{2}$ zeigt eine ausgeprägte Frequenzabhängigkeit und verläuft genauso effizient wie die Glättung mit PC. Im Unterschied zu dem Polymer ist die intrinsische Rauigkeit von $\mathrm{ZrO}_{2}$ mit 0,1 nm (auch für Schichten von über $1 \mu \mathrm{m}$ Dicke) allerdings deutlich geringer, so dass eine Glättung bis zu diesem Wert möglich sein sollte. Im Experiment blieb jedoch eine Endrauigkeit von 0,3 nm übrig, die auf langwellige Strukturen des Substrats zurückzuführen ist, die noch nicht ausgeglättet werden konnten. Die normalen Wellenstrukturen waren bei etwa $200 \mathrm{~nm} \mathrm{ZrO}_{2}$ verschwunden. Genauere Analysen der spektralen Leistungsdichte ergaben, dass wieder Mechanismen der Ordnung 2 und 4 für die Glättung verantwortlich sind. Die Grenzwellenlänge für die Dominanz der Mechanismen lag auf glatten Substraten bei etwa $110 \mathrm{~nm}$, bei den gewellten Substraten etwas niedriger bei etwa $70 \mathrm{~nm}$. Am Beispiel dieses Materials wurde die Glättung der Wellenstrukturen mit dem theoretisch vorhergesagten Ablauf der Glättung verglichen. Der frequenzabhängige Teil konnte gut mithilfe der theoretischen Gleichungen simuliert werden, allerdings zeigen die Experimente zusätzlich noch eine scheinbar frequenzunabhängige Komponente der Glättung, die von der Theorie nicht beschrieben wird. 
Auch mit PMMA-Filmen können Oberflächen gut ausgeglättet werden. Die Eigenrauigkeit des Polymers beträgt 0,3 nm auf glattem Si. Im Unterschied zu den bereits diskutierten Materialien ist der Glättungsvorgang hier allerdings etwas komplizierter, da zunächst aufgrund von Entnetzung sehr hohe Inseln gebildet werden, was Rauigkeiten von bis zu $10 \mathrm{~nm}$ erzeugt. Wenn die Schichten geschlossen sind, fängt das Material an sich glattzuziehen, was interessanter Weise ebenfalls als frequenzabhängiger Prozess identifiziert wurde. Zur Ausglättung dieser selbst erzeugten Rauigkeiten wird eine deponierte Schichtdicke von etwa $200 \mathrm{~nm}$ benötigt. Der Prozess der Entnetzung ist umso stärker je rauer die Substrate sind, allerdings hat dies auf die hier verwendeten Si-Wellen nur sehr geringe Auswirkungen. Die benötigte Schichtdicke zur vollständigen Glättung liegt nur etwas höher als bei glatten Substraten. Da die Wellen durch sehr viel höhere Frequenzen (kleinere Oberflächenstrukturen) charakterisiert sind als die PMMA-Inseln, läuft der Glättungsprozess im Fall von PMMA auf ganz anderen Größenskalen ab. Dies hat zur Folge, dass im Unterschied zur Glättung mit $\mathrm{ZrO}_{2}$ auch langwelligere Strukturen auf den Substraten mitgeglättet werden, ohne dass dafür dickere Schichten notwendig wären.

Im ersten Teil der Arbeit hat sich also gezeigt, dass eine effiziente Oberflächenglättung durch die Deposition unterschiedlicher dünner Schichten möglich ist. Für eine noch genauere Untersuchung der Prozesse während der Glättung und den detaillierten Vergleich mit den Vorhersagen der Theorie erwiesen sich allerdings die dominanten Frequenzen der Wellenstrukturen als störend. Daher wurde im zweiten Teil der Arbeit die Glättung durch $\mathrm{ZrO}_{2}$ systematisch auf statistischen Oberflächenstrukturen untersucht. Dazu wurden geschlossene, 44 nm dicke Ag-Schichten mit einer Rauigkeit von 0,8 nm und einer mittleren Inselgröße von (86 5) $\mathrm{nm}$ als Ausgangsmaterial verwendet. Die statistisch raue Oberflächenstruktur bewirkt, dass die spektralen Leistungsdichten gleichmäßig abfallen, so dass sich Veränderungen genauer beobachten lassen. Während des Glättungsvorgangs beobachtet man mit zunehmender Schichtdicke zunächst das Verschwinden scharfer Kanten und kleiner Strukturen, dann eine Zunahme der mittleren Hügelgröße, also wiederum eine frequenzabhängige Glättung. Die Analyse der spektralen Leistungsdichte zeigt wie erwartet, dass zunächst der Bereich hoher Frequenzen absinkt, während tiefe Frequenzen auf der Oberfläche bestehen bleiben. Bei diesen Substraten lässt sich nun in der Tat beobachten, dass in den Spektren, wie von der Theorie vorhergesagt, zwei Abknickpunkte auftreten, die den Bereich charakterisieren, der gerade geglättet wird. 
Mit zunehmender Schichtdicke verschiebt sich dieser Bereich sukzessive zu niedrigeren Frequenzen und hinterlässt das geglättete Spektrum. Im Fall von $\mathrm{ZrO}_{2}$ auf Ag hat er eine Breite von etwa $40 \mathrm{~nm}$, die sich auch mit zunehmender Schichtdicke, d.h. fortschreitendem Glättungsprozess, nicht ändert. Diese Vorgänge konnten auch theoretisch simuliert und den Messergebnissen zugeordnet werden. Für die Mechanismen ergab sich, dass bis zu einer Strukturgröße von $40 \mathrm{~nm}$ Diffusion der vorherrschende Mechanismus ist, darüber Sputtererosion bzw. ballistisches Glätten. Im Unterschied zu den bisher beobachteten Glättungsprozessen nimmt die Oberflächenrauigkeit jedoch bei kleinen $\mathrm{ZrO}_{2}-$ Schichtdicken zunächst aufgrund der Bildung amorpher $\mathrm{ZrO}_{2}$-Inseln zu. Ab einer deponierten Schichtdicke von $30 \mathrm{~nm}$ setzt die Glättung ein und die Rauigkeit nimmt mit $d_{\mathrm{ZrO}_{2}}^{-0,5}$ ab, was wieder die Vorhersagen der Theorie bestätigt. Die dickste deponierte Schicht von etwa $1 \mu \mathrm{m}$ hinterlässt eine Rauigkeit von $0,2 \mathrm{~nm}$, ist aber nicht ausreichend, um die Oberflächenstrukturen komplett zu glätten. Man kann anhand der Rauigkeitsanalyse abschätzen, dass dafür eine Schichtdicke von etwa $4 \mu \mathrm{m}$ notwendig wäre.

Um die Auswirkungen der frequenzabhängigen Glättung besser zu verstehen und zu demonstrieren, wurde schließlich die Glättung in Abhängigkeit von der Strukturgröße auf der Oberfläche betrachtet. Hierfür wurden als Substrat Ag-Schichten unterschiedlicher Insel- bzw. Korngröße verwendet, als Glättungsmaterial wieder $\mathrm{ZrO}_{2}$, diesmal allerdings eine festgelegte Schichtdicke von $44 \mathrm{~nm}$. Bei den geschlossenen Ag-Strukturen beeinflusst nun nur noch die Strukturgröße auf der Oberfläche den Grad der Glättung. Je kleiner sie sind, desto vollständiger kann mit einer festen Menge $\mathrm{ZrO}_{2}$ geglättet werden und desto geringere Schichtdicken wären notwendig, um eine vollständige Glättung zu erzielen. Ansonsten hat sich gezeigt, dass die Glättung stark abhängig ist von dem AgBedeckungsgrad der Si-Oberfläche. Bei dünnen Ag-Schichten bis 1,5 nm Dicke liegen getrennte Inseln auf dem Substrat vor. Hier wächst das $\mathrm{ZrO}_{2}$ hauptsächlich auf der glatten Si-Oberfläche unbeeinflusst von den Ag-Inseln und glättet die teilweise sehr großen Rauigkeiten von bis zu $4 \mathrm{~nm}$ bereits nach der Deposition von $44 \mathrm{~nm}$ Material vollständig. Die hinterlassene Oberfläche entspricht der einer reinen $\mathrm{ZrO}_{2}$-Schicht auf Si. Dies spricht dafür, dass die Ausbildung einer $\mathrm{ZrO}_{2}$-Ag-Grenzfläche energetisch ungünstig ist. Das auf den Ag-Inseln deponierte Material diffundiert großteils in die Täler, so dass die Inseln überwachsen werden können ohne einen Beitrag zur Endrauigkeit der Oberfläche zu 
leisten. Sobald die Ag-Schichten koaleszieren und perkolieren, wird der Anteil freier SiOberfläche immer geringer, was dazu führt, dass das vollständige Überwachsen durch die endliche Diffusionslänge von $\mathrm{ZrO}_{2}$ auf Ag verhindert wird. Es bilden sich zunächst $\mathrm{ZrO}_{2}$ Inseln auf Ag, danach geschlossene Schichten.

Insgesamt konnte also in der Arbeit gezeigt werden, dass es möglich ist, mittels der Deposition dünner Schichten raue Oberflächen effektiv zu glätten. Weiterhin konnten die von der Glättungstheorie gemachten Vorhersagen in vielerlei Hinsicht bestätigt werden: Die für eine effiziente Glättung geeigneten Materialien müssen zum einen eine geringe Eigenrauigkeit besitzen, zum anderen bei allen Frequenzen Glättungsmechanismen aufweisen, die die vorhandenen rauen Strukturen schnell glätten. Während Ag und C diese Kriterien nicht erfüllen, ist es bei den Polymeren PC und PMMA und dem Oxid $\mathrm{ZrO}_{2}$ tatsächlich gelungen, die auf dem Substrat vorhanden Rauigkeiten zu eliminieren und sehr glatte Oberflächen zu erzeugen. Die erzeugten Endrauigkeiten lagen zwischen 0,4 nm und 0,2 $\mathrm{nm}$ je nach Eigenrauigkeit des Materials, die notwendige Materialmenge zwischen 70 und etwa $200 \mathrm{~nm}$. Des Weiteren konnte die aus der Theorie bekannte frequenzabhängige Glättung experimentell im Detail nachvollzogen und mithilfe der theoretischen Gleichungen simuliert werden, allerdings beobachtet man experimentell noch einen frequenzunabhängigen Beitrag zur Glättung, der von der Theorie nicht erklärt werden kann. Benutzt man PLD als Dünnschichtmethode, ist vor allem Diffusion für kleinere Strukturgrößen der dominante Glättungsmechanismus, während bei größeren Strukturen Sputtererosion und ballistisches Glätten vorherrschend sind. Neben der mittleren Strukturgröße, die die Schnelligkeit der Glättung beeinflusst, spielen auch die verwendeten Materialkombinationen eine wichtige Rolle. So konnte gezeigt werden, dass vereinzelte Ag-Inseln auf einem Si-Substrat deutlich schneller geglättet werden können als geschlossene Ag-Schichten vergleichbarer Strukturgröße, da die Ausbildung einer $\mathrm{ZrO}_{2}$ Ag-Grenzfläche energetisch ungünstig ist.

Interessanterweise ist die effektive Glättung von rauen Oberflächen folglich durch die Deposition sowohl von oxidischen Materialien als auch von Polymeren möglich. Wesentlich ist hierbei, dass die Glättung in allen Fällen frequenzabhängig verläuft, so dass die auf der Oberfläche vorhandenen Strukturgrößen eine entscheidende Rolle spielen, und die erreichbare Endrauigkeit abhängig von der Eigenrauigkeit der Glättungsmaterialien ist. 


\section{Literaturverzeichnis}

[Ande84] M.P. Anderson, D.J. Srolovitz, G.S. Grest, P.S. Sahni: Computer simulation of grain growth - I. Kinetics; Acta metall. 32, 783 (1984)

[Bara95] A.-L. Barabási, H.E. Stanley: Fractal Concepts in Surface Growth; Cambridge University Press, Cambridge, UK, 1995

[Barr69] W.P. Barr: The production of low scattering dielectric films using rotating vane particle filtration; J. Phys. E 2, 1112 (1969)

[Baue06] E.F. Bauer: Statik und Kinetik der Entnetzung ultradünner Polymerfilme; Dissertation, TU-München (2006)

[Binn86] G. Binnig, C.F. Quate, Ch. Gerber: Atomic Force Microscope; Phys. Rev. Lett. 56, 930 (1986)

[Brad88] R.M. Bradley, J.M.E. Harper: Theory of ripple topography induced by ion bombardment; J. Vac. Sci. Technol. A 6, 2390 (1988)

[Chri88] F.E. Christensen, A. Hornstrup, H.W. Schnopper: Surface correlation function analysis of high resolution scattering data from mirrored surfaces obtained using a triple-axis x-ray diffractometer; Appl. Opt. 27, 1548 (1988)

[Chri94] D.B. Chrisey, G.K. Hubler (editors): Pulsed laser deposition of thin films; Wiley Interscience Publication, New York (1994)

[Cull01] B.D. Cullity, S.R. Stock: Elements of x-ray diffraction; Pearson/Prentice Hall Publishing Company, New Jersey (2001)

[Dyer03] P.E. Dyer: Excimer laser polymer ablation: twenty years on; Appl. Phys. A 77, 167 (2003)

[Easo07] R. Eason: Pulsed laser deposition of thin films; Wiley, New Jersey (2007) 
8 Literaturverzeichnis

[Eise01] C. Eisenmenger-Sittner: Surface evolution of polycrystalline Al films deposited on amorphous substrates at elevated temperatures; J. Appl. Phys. 89, 6085 (2001)

[Erle97] J.D. Erlebacher, M.J. Aziz: Morphological equilibration of ripppled and dimpled crystal surfaces: the role of terrace-width fluctuations; Surf. Sci. 374, 427 (1997)

[Facs99] S. Facsko, T. Dekorsky, C. Koerdt, C. Trappe, H. Kurz, a. Vogt, H.L. Hartnagel: Formation of ordered nanoscale semiconductor dots by ion sputtering; Science 285, 1551 (1999)

[Fähl97] S. Fähler, M. Störmer, H.U. Krebs: Origin and avoidance of droplets during laser ablation of metals; Appl. Surf. Sci. 109/110, 433 (1997)

[Fang97] S.J. Fang, S. Haplepete, W. Chen, C.R. Helms, H. Edwards: Analyzing atomic force microscopy images using spectral methods; J. Appl. Phys. 82, 5891 (1997)

[Faup02] J. Faupel, H.U. Krebs, A. Käufler, Y.Luo, K. Samwer, S. Vitta: Giant magnetoresistance in laser deposited Permalloy/Ag multilayers; J. Appl. Phys. 92, $1171(2002)$

[Faup05] J. Faupel: Wachstum von metallischen Nanoclustern auf Polymeroberflächen; Dissertation, Uni Göttingen (2005)

[Fros00] F. Frost, A. Schindler, F. Bigl: Roughness evolution of ion sputtered InP surfaces: pattern formation and scaling laws; Phys. Rev. Lett. 85, 4116 (2000)

[Fuch09] B. Fuchs, interne Mitteilungen

[Full92] E.E. Fullerton, D.M. Kelly, J. Guimpel, I.K. Schuller, Y. Bruynseraede: Roughness and Giant Magnetoresistance in Fe/Cr Superlattices; Phys. Rev. Lett. 68, 859 (1992)

[Hans88] S.G. Hansen, T.E. Robitaille: Formation of polymer films by pulsed laser evaporation; Appl. Phys. Lett. 52, 81 (1988)

[Herr50] C. Herring: Effect of change of scale on sintering phenomena; J. Appl. Phys. 21, 301 (1950)

[Herw05] C. Herweg: Selbstorganisation magnetischer Nanopartikel auf facettierten SaphirSubstraten, Universität Göttingen, Dissertation (2005)

[Hoff76] R.W. Hoffman: Stresses in thin films: The relevance of grain boundaries anad impurities; Thin Solid Films 34, 185 (1976)

[Holz92] B. Holzapfel, B. Roas, L. Schultz, P. Bauer, G. Saemann-Ischenko: Off-axis laser deposition of $\mathrm{YBa}_{2} \mathrm{Cu}_{3} \mathrm{O}_{7-\delta}$ thin films; Appl. Phys. Lett. 61, 3178 (1992) 
8 Literaturverzeichnis

[Kell85] R. Kelly, J.J. Cuomo, P.A. Leary, J.E. Rothenberg, B.E. Braren, C.F. Aliotta: Laser sputtering Part I. on the existence of rapid laser sputtering at $193 \mathrm{~nm}$; Nucl. Instrum. Meth. Phys. Res. B 9, 329 (1985)

[Kreb07] H.U. Krebs: Pulsed laser deposition of metals, section III, chapter 16 in R. Eason: Pulsed laser deposition of thin films; Wiley, New Jersey (2007)

[Kreb97] H.U. Krebs: Characteristic properties of laser deposited metallic systems; J. of NonEqu. Proc. 10, 3 (1997)

[Li03] D. Li, Y. Chen, Y.-W. Chung, F.L. Freire: Metrology of 1-10 nm thick $C_{x}$ films: Thickness, density, and surface roughness measurements; J. Vac. Sci. Technol. A 21, L19 (2003)

[Liu02] Z.-J. Liu, N. Jiang, Y.G. Shen, Y.W. Mai: Atomic force microscopy study of surface roughening of sputter-deposited TiN thin films; J. Appl. Phys. 92, 3559 (2002)

[Löse06] B. Lösekrug: Energiedichteabhängigkeit der Eigenschaften laserdeponierter Poly(methyl methacrylat)-Schichten und -Kompositmaterialien; Diplomarbeit, Uni Göttingen (2006)

[Löse07] B. Lösekrug, A. Meschede, H.U. Krebs: Pulsed laser deposition of smooth poly (methyl methacrylate) films at 248 nm; Appl. Surf. Sci. 254, 1312 (2007)

[Lüth95] H. Lüth: Surfaces and interfaces of solid materials, Springer, Berlin (1995)

[Mart87] Y. Martin, C.C. Williams, H.K. Wickramasinghe: Atomic force microscope-force mapping and profiling on a sub 100-Å scale; J. Appl. Phys. 61, 4723 (1987)

[Mayr01] S.G. Mayr, R.S. Averback: Surface smoothing of rough amorphous films by irradition-induced viscous flow; Phys. Rev. Lett. 87, 196106 (2006)

[Merk98] V.I. Merkulov, D.H. Lowndes, G.E. Jellison, Jr., A.A. Puretzky, D.B. Geohegan: Structure and optical properties of amorphous diamond films prepared by ArF laser ablation as a function of carbon ion kinetic energy; Appl. Phys. Lett. 73, 2591 (1998)

[Merk99] V.I. Merkulov, D.H. Lowndes, L.R. Baylor: Field-emission studies of smooth and nanostructured carbon films; Appl. Phys. Lett. 75, 1228 (1999)

[Mill90] M.S. Miller, W.L. Skelcher, P.B. Narayan, G.T. Swami, J. Crist, R.L. Peterson, R.R. Thomas, H.F. Erskine: The effect of deposition parameters on the wear properties of yttria-stabilized zirconia overcoats; J. Appl. Phys. 67, 4872 (1990) 
8 Literaturverzeichnis

[Mose05] M. Moseler, P. Gumbsch, C. Casiraghi, A.C. Ferrari, J. Robertson: The ultrasmoothness of diamond-like carbon surfaces; Science 309, 1545 (2005)

[Mull57] W.W. Mullins: Theory of thermal grooving; J. Appl. Phys. 28, 333 (1957)

[Mull59] W.W. Mullins: Flattening of a nearly plane solid surface due to capillarity; J. Appl. Phys. 30, 77 (1959)

[Nix99] W.D. Nix, B.M. Clemens: Crystallite coalescence: a mechanism for intrinsic stresses in thin films; J. Mater. Res. 14, 3467 (1999)

[Okab05] J. Okabayashi, S. Toyoda, H. Kumigashira, M. Oshima, K. Usuda, M. Niwa, G.L. Liu: Crystallization and chemical structures with annealing in $\mathrm{ZrO} 2$ gate insulators studied by photoemission spectroscopy and x-ray absorption spectroscopy; J. Vac. Sci. Technol. A23, 1554 (2005)

[Orch62] S.E. Orchard: On surface levelling in viscous liquids and gels; Appl. Sci. Res. A 11, $451(1962)$

[Papp92] D.L. Pappas, K.L. Saenger, J.J. Cuomo, R.W. Dreyfus: Characterization of laser vaporization plasmas generated for the deposition of diamond-like carbon; J. Appl. Phys. 72, 3966 (1992)

[Parr54] L.G. Parratt: Surface studies of solids by total reflection of x-rays; Phys. Rev. 95, 359 (1954)

[Rehs01] N. Rehse, C. Wang, M. Hund, M. Geoghegan, R. Magerle, G. Krausch: Stability of thin polymer films on a corrugated substrate; Eur. Phys. J. E 4, 69 (2001)

[Röde05] J. Röder: Beeinflussung des Wachstums von Ag auf PMMA, Universität Göttingen, Diplomarbeit (2005)

[Röde08] J. Röder, J. Faupel, H.U. Krebs: Growth of polymer-metal nancomposites by pulsed laser deposition; Appl. Phys. A 93, 863 (2008)

[Sava91] D.E. Savage, J. Kleiner, N. Schimke, Y.-H. Phang, T. Jankowski, J. Jacobs: Determination of roughness correlations in multilayer films for $x$-ray mirrors; $\mathrm{J}$. Appl. Phys. 69, 1411 (1991)

[Sevc73] N.J. Sevchik: Growth instabilities in the deposition of amorphous films; J. NonCryst. Solids 12, 141 (1973)

[Sing90] R.K. Singh, D. Bhattacharya, J. Narayan: Subsurface heating effects during pulsed laser evaporation of materials; Appl. Phys. Lett. 57, 2022 (1990) 
8 Literaturverzeichnis

[Süsk06] E. Süske, T. Scharf, T. Junkers, M. Buback, H.U. Krebs: Mechanism of poly(methyl methacrylate) film growth during pulsed laser deposition; J. App. Phys. 100, 014906 (2006)

[Thij92] J.M. Thijssen, H.J.F. Knops, A.J. Dammers: Dynamic scaling in polycrystalline growth; Phys. Rev. B 45, 8651 (1992)

[Tong94] W.M. Tong, R.S. Williams: Kinetics of Surface Growth: Phenomenology, Scaling, and mechanisms of smoothening and roughening; Annu. Rev. Phys. Chem. 45, 401 (1994)

[Tsel01] A. Tselev, A. Gorbunov, W. Pompe: Cross-beam pulsed laser deposition: General characteristics; Rev. Sci. Instr. 72, 2665 (2001)

[Vaut07] S. Vauth: Glättungsmechanismen beim Ionenbeschuss rauer amorpher Oberflächen, Universität Göttingen, Dissertation (2007)

[Vaut08] S. Vauth, S.G. Mayr: Ion bombardment induced smoothing of amorphous metallic surfaces: experiment versus computer simulations; Phys. Rev. B 77, 155406 (2008)

[Vill91] J. Villain: Continuum models of crystal growth from atomic beams with and without desorption; J. Phys. I 1, 19 (1991)

[Voev00] A.A. Voevodin, J.G. Jones, J.S. Zabinski: Characterization of $\mathrm{ZrO}_{2} / \mathrm{Y}_{2} \mathrm{O}_{3}$ laser ablation plasma in vacuum, oxygen and argon environments; J. Appl. Phys. 88, 1088 (2000)

[Voev01] A.A. Voevodin, J.G. Jones, J.S. Zabinski: Structure control of pulsed laser deposited $\mathrm{ZrO}_{2} / \mathrm{Y}_{2} \mathrm{O}_{3}$ films; J. Vac. Sci. Technol. A 19, 1320 (2001)

[West94] K.L. Westra, D.J. Thomson: Effect of tip shape on surface roughness measurements from atomic force microscopy images of thin films; J. Vac. Sci. Technol. B13, 344 (1994)

[Wind98] D.L. Windt: IMD-Software for modelling the optical properties of multilayer films; Computers in Physics 12, 360 (1998)

[Zhon93] Q. Zhong, D. Inniss, K. Kjoller, V.B. Elings: Fractured polymer/silica fiber surface studied by tapping mode atomic force microscopy; Surf. Sci. Lett. 290, L699 (1993)

[Zibe08] B. Ziberi, F. Frost, M. Tartz, H. Neumann, B. Rauschenbach: Ripple rotation, pattern transitions, and long range ordered dots on silicon by ion beam erosion; Appl. Phys. Lett. 92, 063102 (2008) 


\section{Danksagung}

Ich möchte mich hiermit ganz herzlich bei allen bedanken, ohne die es nicht möglich gewesen wäre, diese Arbeit zu schreiben!

An erster Stelle möchte ich mich bei Prof. Dr. Hans-Ulrich Krebs bedanken für die Möglichkeit, in seiner Gruppe diese Arbeit zu schreiben. Sein stetes Interesse und viele intensive Gespräche und Diskussionen haben wesentlich zum Gelingen der Arbeit beigetragen. Über die fachliche Zusammenarbeit hinaus ist auch sein persönliches Interesse an uns erwähnenswert, das die Atmosphäre in der Lasergruppe wesentlich aufgelockert und ein gutes Arbeitsklima geschaffen hat.

Ich bedanke mich auch ganz besonders bei den Mitgliedern meiner Arbeitsgruppe für die gute Zusammenarbeit und viele angeregte Diskussionen im Labor, bei den Gruppensitzungen und den Frühstücksrunden im Nachbarbüro. Aber auch über das fachliche hinaus bedanke ich mich für entspannende Gespräche am See, KickerWettkämpfe und die vielen erholsamen Mittagspausen, die wir zusammen verbracht haben. Angefangen bei Conny Mewes, Andreas Meschede, Britta Fuchs, Tobias Liese und Susanne Seyffarth, bis hin zu unserem externen Doktoranden Frank Barkusky und unserem Gruppenmitglied in spe Felix Schlenkrich. Nicht unerwähnt bleiben sollen auch die jetzt Ehemaligen Sebastian Dreyer und Thorsten Scharf. Unvergesslich bleiben viele aufmunternde Gespräche über Gott und die Welt, so manche gemeinsamen (sportlichen) Betätigungen und nicht zuletzt viele Tagungen, die wir gemeinsam verbracht und immer zu einem tollen Erlebnis gemacht haben. Hier bleiben so einige DPGs und vor allem eine wunderschöne Woche bei der COLA auf Teneriffa in Erinnerung mit unglaublich gutem Essen (tollen Nachtisch-Kreationen), sehr spannenden fachlichen Beiträgen und interessanten PhysikerInnen, die wir kennengelernt haben.

Allen Mitgliedern des Sonderforschungsbereichs 602 danke ich für so manche Diskussionen und die gute Zusammenarbeit. Insbesondere möchte ich mich bei Christian 
Vree, Dr. Sebastian Vauth und Prof. Dr. Stefan Mayr bedanken für die Einführung ins AFM, sowie für viele intensive und hilfreiche Diskussionen über Rauigkeiten und allem was dazugehört. Des Weiteren danke ich Arne Schäfer, Sven Krüger und Prof. Dr. Tim Salditt für ihre Zusammenarbeit.

Mein Dank gilt auch allen Mitarbeitern des Instituts für Materialphysik für das nicht nur im zweiten Stock - angenehme Arbeitsklima und ihre Unterstützung bei den kleineren und größeren Problemen des Arbeitsalltags sowie diverse Einweisungen in die verschiedenen Geräte.

Ich bedanke mich des Weiteren bei Dr. B. Ziberi und Dr. F. Frost vom LeibnizInstitut für Oberflächenmodifizierung e.V. in Leipzig für die Präparation der SiWellenstrukturen.

Bei meinen Freunden in und außerhalb des Instituts, die mich durch die Höhen und Tiefen dieser Zeit begleitet haben, bedanke ich mich für viele schöne, gemeinsam verbrachte Stunden, die für einen angenehmen Ausgleich zur Physik gesorgt haben.

Nicht zuletzt bedanke ich mich ganz besonders bei meinen Eltern, die mir dieses Studium ermöglicht haben, und bei meiner Schwester, der wahrscheinlich tollsten Schwester der Welt. Sie waren in allen Lebenslagen für mich da, hatten ein offenes Ohr für alle Probleme (und eine Lösung für die meisten) und haben mir die beste Unterstützung gegeben, die ich mir wünschen konnte! Ohne sie wäre dies alles nicht möglich gewesen. 


\section{Lebenslauf}

\section{Angaben zur Person:}

Name:

Geburtstag:

Geburtsort:

Familienstand:

Staatsangehörigkeit:

\section{Schulbildung:}

1987 - 1991

1991 - 2000

2000

\section{Studium:}

$07.2000-08.2000$

$10.2000-07.2002$

05.07.2002

10.2002

$08.2003-10.2003$

12.2005

01.2006
Johanna Röder

25.03.1981

Kassel

ledig

Deutsch

Grundschule Harleshausen, Kassel

Friedrichsgymnasium Kassel

Abitur

Praktikum am Forschungszentrum Jülich, Institut für Plasmaphysik (IPP)

Grundstudium in Physik an der Georg-August-Universität Göttingen

Vordiplom in Physik

Hauptstudium in Physik an der Georg-August-Universität Göttingen

Praktikum am Institut de Ciència de Materials de Barcelona (ICMAB), CSIC, Spanien

Diplom in Physik, Thema der Diplomarbeit: „Beeinflussung des Wachstums von Ag auf PMMA“ angefertigt am Institut für Materialphysik in der Arbeitsgruppe von Prof. Dr. H.-U. Krebs

Beginn der Dissertation am Institut für Materialphysik in der Arbeitsgruppe von Prof. Dr. H.-U. Krebs, wissenschaftliche Mitarbeiterin im Sonderforschungsbereich 602 „Komplexe Strukturen in kondensierter Materie von atomarer bis mesoskopischer Skala“ 